TURBULENT BOUNDARY-LAYER HEAT TRANSFER FROM RAPIDLY ACCELERATING FLOW OF ROCKET COMBUSTION GASES AND OF HEATED AIR

\author{
D. R. Bartz
}
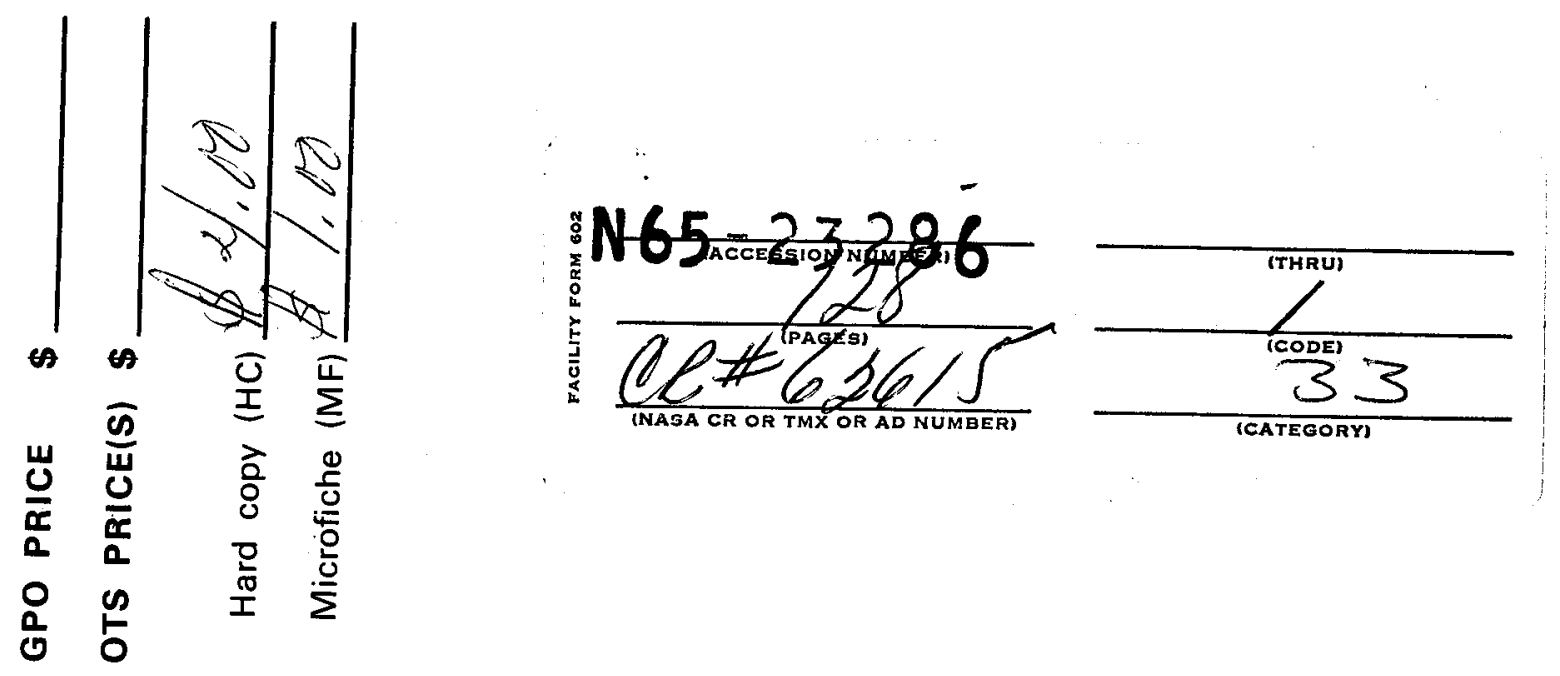

Jet Propulsion Laboratory California Institute of Technology

Pasadena, California

December, 1963 
FOOTNOTE

This Chapter is contributed jointly by the author and the Jet Propulsion Laboratory, California Institute of Technology, where portions of the work were done under Department of the Army Contract No. DA-04-495-Ord 18 and National Aeronautics and Space Administration Contract Nos. NASw-6 and NAS 7-100. 


\section{CONTENTS}

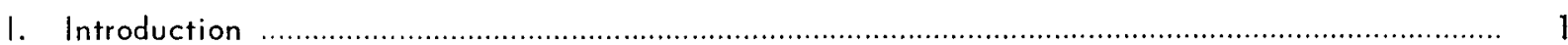

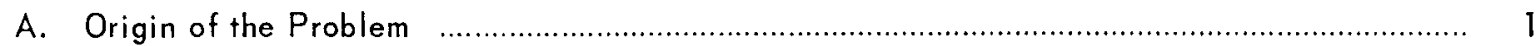

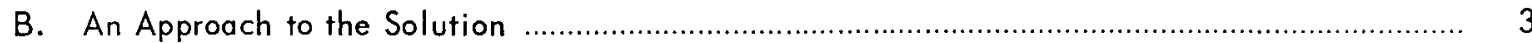

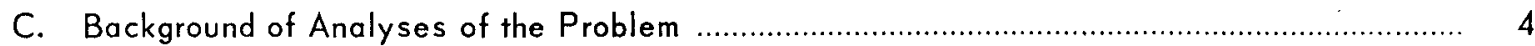

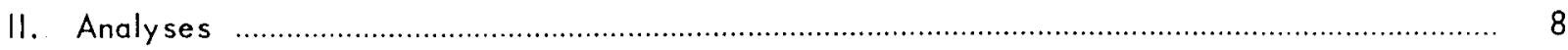

A. Integral Momentum and Energy Equation Solution............................................................. 8

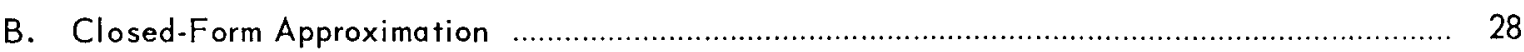

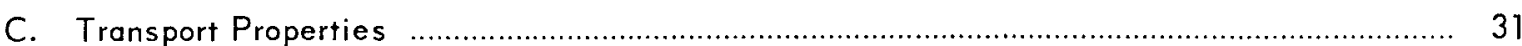

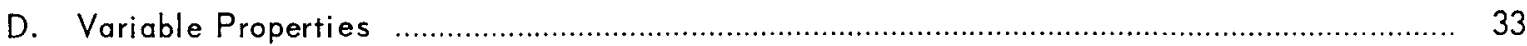

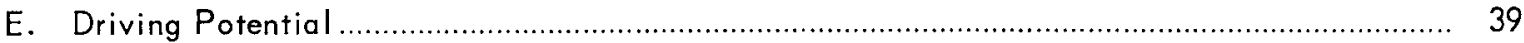

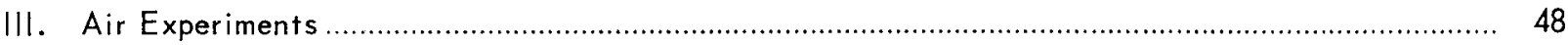

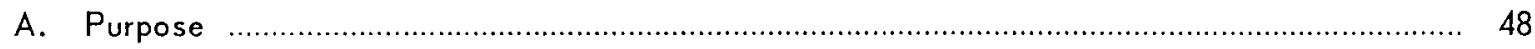

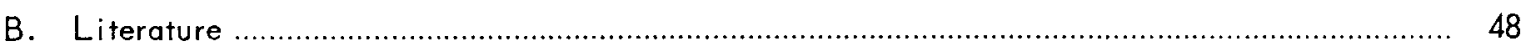

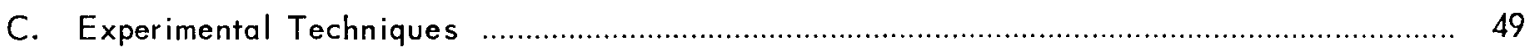

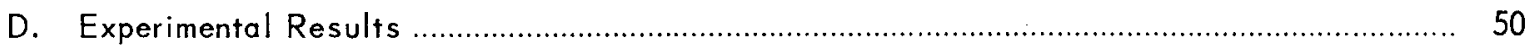

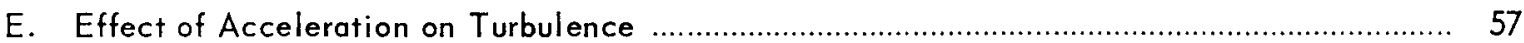

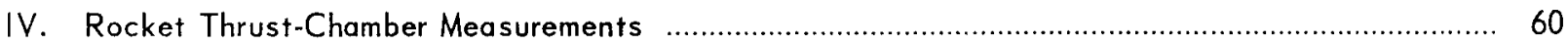

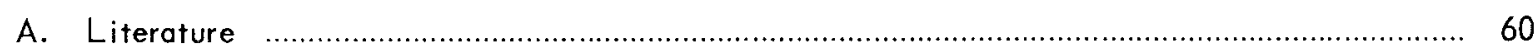

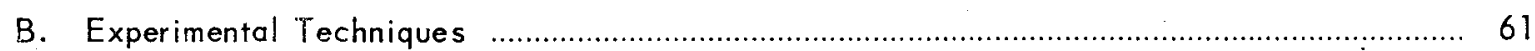

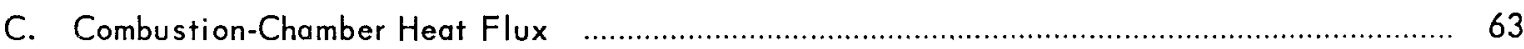

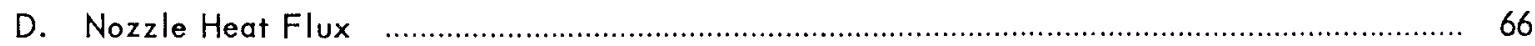

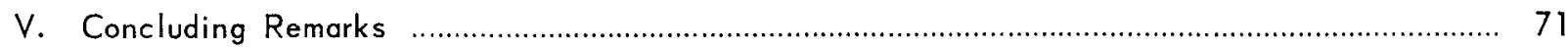

Appendixes

A. Coles' Skin-Friction Coefficient and von Kármán Form of Reynolds Analogy ...................... 74 


\section{CONTENTS (Cont"d)}

B. Boundary-Layer Shape Parameter Evaluation ......................................................................... 77

C. Boundary-Layer Thicknesses and Integral Equations for Thick Boundary Layers ................ 80

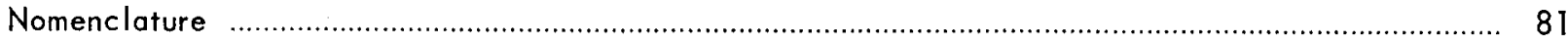

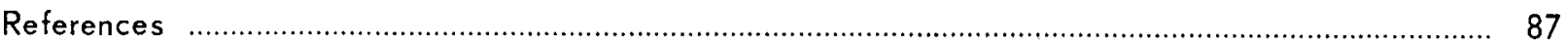




\section{INTRODUCTION}

\section{A. Origin of the Problem}

Fver since the development of rocket engines for practical application, there has been a recognized need to predict the heat transfer from the combustion gases to the walls of both the combustion chamber and the nozzle. Since, in the early days, these walls were generally constructed of materials with negligible strength above about $1500^{\circ} \mathrm{F}$ and had to contain gases at pressures of a few hundred pounds per square inch and temperatures of 4000 to $5000^{\circ} \mathrm{F}$, the consequence of underdesigned wall-protection provisions was a serious local wall failure and, frequently, a blown-up engine; the consequence of grossly overdesigned wall-protection provisions was excessive pressure drop and weight, or demands of shifts in the engine operating mixture ratio toward lower performance. As a result, the prediction of heat transfer with sufficient accuracy to avoid failures and sacrifices in weight or performance became increasingly important. More recently, chamber pressures of large booster engines have reached the $1000-\mathrm{lb} / \mathrm{inch}^{2}$ level and there are indications that in the foreseeable future these pressures may be doubled. In addition, the use of more energetic propellants has driven combustion gas temperatures of these engines up near $8000^{\circ} \mathrm{F}$. New trends in smaller en gines for upper stages and for spacecraft have also increased the demand for knowledge of the heat transfer and boundary-layer growth. In the interests of simplicity, or in the absence of sufficient or suitable propellant coolant, use has been made of ablating walls or refractory metal walls cooled by radiation. Although there is no general acceptable theory of the ablation process for heterogeneous materials, it has been shown to be generally related to the he at transfer $(1,2)$. The need for detailed knowledge of the momentum losses or boundary-layer growth in the supersonic part of nozzles has been increased by the trend toward very large expansion ratios, which result in performance gains for space operatio. At some area ratio, depending on the design of the wall, the gains in performance obtained by a still higher area ratio are offset by the added weight of the wall. Hence, it becomes important to ascertain the real performance by assessing the friction losses. Other recent developments such as generating shocks to provide thrust-vector control also require knowledge of boundary-layer development. Thus, the need for knowledge of both heat transfer and boundary-layer development in "rocket thrust chambers" (used commonly to refer collectively to both combustion chamber and nozzle) has expanded with the years during which rocket engine development has evolved. Fortunately, the knowledge available has expanded significantly as well. However, as will become evident from this Chapter, the problem is not "solved." 
One might ask in what way heat transfer and boundary-layer development in rocket thrust chambers are so special that the problem is still not solved after more than a decade of concentrated analysis and experiment. For most flow fields too complex to permit exact solutions, the practice has long been to create a model by making typical, and usually permissible, assumptions such as inviscid core flow, laminar boundary layer or turbulent boundary layer with specified eddy diffusivity, one-dimensional, constant properties, steady flow, etc. 'The deviations of the real flow from the model are accounted for by small correction factors determined by correlating data for the real flow against predictions of the model. The problem with the rocket thrust-chamber flows is that the real flow is characterized by numerous deviations from flow describable by a simple model, and the deviations are not necessarily small. The most significant deviation or complexity is that the free stream flow cannot in general be successfully described in terms of steady, average, one-dimensional flow variables. In particular, the flow in the combustion region (and this may include a substantial portion of the nozzle, depending on propellants and configurations) is frequently characterized by severe large-scale secondary flows, non-linear oscillations, and variable total temperature. Each is characteristic of particular propellants, propellant injectors, operating conditions, and combustion-chamber configurations. As yet, our knowledge of combustion is insufficient either to predict or to control this behavior. Since heat-transfer predictions cannot proceed beyond our ability to describe the fluid dynamics and energy states, it should begin to be evident why prediction of heat transfer and boundary-layer development in rocket thrust chambers is difficult. The next most significant complexity is that the free stream, and hence the boundary-layer flows, are rapidly accelerating; thus, it becomes impossible to neglect axial pressure-gradient terms in the momentum and energy equations. Because of this, it is no longer possible to express the momentum and en ergy equations in similar form, and hence to derive useful analogies between momentum and energy transport except in an heuristic fashion. Another significant complexity is the possible occurrence of chemical reaction in the free stream which leads to an axially varying total temperature or enthalpy, and chemical reaction in the boundary layer due to the recombination of dissociated chemical species. Since most of the chemical reactions in question are exothermic, they can play a pronounced role in modifying the driving potential for heat transfer. A further significant complexity is the fact that for most rocket flows of interest the boundary layer (and probably the free stream as well) is very likely to be turbulent. One arrives at this conclusion by noting the generally very high Reynolds numbers due to very high mass flow rates per unit area, not compensated by unusually high viscosity or small linear dimensions. Furthermore, from the usual free-stream turbulence due to combustion, one would expect boundary-layer transition to occur at unusually 
low Reynolds numbers, outweighing the stabilizing effects of cooling and acceleration. There are still other complexities such as extreme property variations across the boundary layer, an uncertain flow origin, separation due to high ambient pressure "leaking" up the subsonic portion of the boundary layer in the divergent part of the nozzle, etc.

\section{B. An Approach to the Solution}

In the face of a problem with as many complicating elements as described, one must adopt a pragmatic approach such as restricting the analysis to that part of the problem that can be handled (or almost handled with "plausible" assumptions) and then fully recognize the limitations of the result. One can only hope to remove the currently necessary restrictions by deeper specific knowledge of the phenomena involved, to be gained by carefully instrumented and controlled experimentation. Among the currently necessary restrictions in this author's opinion are that (1) the flow to be considered be beyond the region of severe secondary flows due to combustion, (2) the ultimately attainable combustion temperature be established in the free stream, and (3) the engine be operating without significant combustion pressure oscillations. (Research on the effect of pressure and velocity fluctuations such as reported in References 3,4 , and 5 may some day make it possible to avoid the latter restriction.) A further necessary restriction is that chemical recombination in the boundary layer proceed according to local chemical equilibrium conditions or the close equivalent, a diffusion-controlled chemically frozen boundary layer with a catalytic wall. (Theory and experiment of the type described in Reference 6 could make this restriction unnecessary if certain assumptions are made. However, critical experiments, such as reaction rate measurements, that would determine which of such assumptions are plausible have not been made in the rocket engine environment.) Additional restrictions are that the boundary layer be fully turbulent, have some specified thickness at the starting point of the analysis (such as that appropriate to growth in a pipe entrance region of approximately the combustion-chamber length when starting at the nozzle entrance), and that the region of unseparated flow be of primary interest. Thus, it is evident that, in effect, one has either had to ignore the combustion zone or to assume its heat transfer to be no higher than that predicted at the starting point of the analysis. This may or may not be a good assumption, as will be evident later. With these restrictions, it is possible, by making a number of "plausible" assumptions, to predict both boundarylayer development and heat transfer. The "plausible assumptions" and methods for making such predictions are discussed in Section II of this Chapter. To establish the validity of these methods of prediction, it is necessary to compare the predictions with the results of carefully controlled experiments 
in which the existence of the restricted conditions assumed is assured. This is done to the limit of the availability of such experimental results in Section III. Finally, one must compare the predictions with results from measurements of real rocket thrust-chamber flows under a wide variety of conditions to determine to what extent the real flows deviate from that assumed in the model. Such comparisons are made, also to the limit of availability of experimental results, in Section IV.

\section{Background of Analyses of the Problem}

Before proceeding with a current version of the analysis of the problem specified in B above, it might be of value to trace the stages of evolution of earlier analyses of this problem. Initially, for want of better information on turbulent boundary layers in nozzles, the classic turbulent pipe-flow heattransfer correlation equations of McAdams and of Colburn (7) were applied by considering the nozzle flow to be a series of fully developed turbulent pipe flows. Each point in the nozzle was assumed to have been preceded by a very long pipe of the local diameter of interest. Because this approach seemed to work well (although there was a very limited amount of local heat-flux data with which to compare it), there was a tendency to lose sight of the fact that the flow was by no means fully developed in the sense of boundary layers extending to the flow axis of symmetry. Not satisfied with the apparent incompatibility of the actual flow regime with that which served as the basis for the analytical prediction, several workers attempted to solve the nozzle heat-transfer problem from a boundary-layer viewpoint making use of the integral momentum and energy equations $(8,9)$. The essential difference between the nozzl e problem and most of the turbulent boundary-layer analytical treatments then published was the necessity for retaining the pressure gradient terms in the equations of motion. The way was already partially paved since the momentum transfer problem had been solved by approximate methods under the impetus of computing boundary-layer corrections to the contours of supersonic wind-tunnel nozzles $(10,11,12)$. Experimental results were found to agree quite satisfactorily with predicted boundary-layer thicknesses.

The new extension achieved in References 8 and 9 was the handling of the heat-transfer, as well as the momentum-transfer problem. Numerical results from the approximate solutions obtained were found to agree reasonably well with limited experimental data then available and with predictions made on the pipe-flow basis except in nozzl e-entrance regions. Here, the possibility of extremely thin boundary layers was shown to result in correspondingly high local heat fluxes, as should be expected. Since the boundary-layer approach was considered physically valid, it was expected that this method of analysis 
would serve the purpose of making reasonably accurate predictions of convective heat transfer in supersonic nozzles. It was anticipated that the analysis would be improved as further basic knowledge of the skin friction and heat transfer in accelerating turbulent boundary layers was obtained.

In that era, which preceded the wide availability of high-speed computers and the practice of sharing programs among organizations, it soon became evident that a method of an alysis requiring the solution of a pair of differential equations with coefficients varying in accordance with each particular nozzle contour could not be used very widely. Consequently, a closed-form equation which could be hand-computed and which closely approximated the results of the boundary-layer analysis for a particular typical nozzle configuration and typical initial boundary-layer conditions was sought and found. It was clearly evident from the boundary-layer heat-transfer calculations (9) that the dominant parameter in the variation of the local heat-transfer coefficient was the local mass flux raised to the eight-tenths power. This suggested the possibility of again utilizing the dimensionless parameter approach employing Reynolds number, Prandtl number, and Nusselt number. Such an approach, however, raised the question of the characteristic length dimension to be used. A review of the boundary-layer development in a nozzle (9, Fig. 3) showed that the local boundary-layer thickness varied in a systematic relationship with the local diameter, suggesting that the local diameter be used as the characteristic length. When the diameter was so employed, the dimensionless equation looked identical in form to the McAdams and Colburn pipeflow equations with a proportionality constant to be determined, thus accounting for the early success of such equations when applied to nozzle flow. The proportionality constant was determined by fitting the closed-form equation to the boundary-layer heat-transfer calculations at the throat for a particular case, estimated to be reasonably typical of then current rocket nozzles. Some additional minor modifications resulting from variable properties considerations, and effects of throat radius of curvature were deduced from the boundary-layer results and applied to the closed-form equation, giving the result published in Reference 13.

This closed-form equation served its purpose quite satisfactorily until, with time, several changes occurred. First, with the increasing availability of high-speed computers, the compromises inherent in such an equation were no longer necessary; an exact solution, to the extent permitted by knowledge of the turbulent boundary layer, could be computed almost as readily as the closed-form equation. Second, nozzles of interest were no longer restricted to simple conical convergent-divergent nozzles in which local flow conditions were easily expressible in terms of local area ratio. So-called "bell" nozzles resulted in regions of severely turned flow, in which the mass flux near the wall was considerably different from that 
predicted by one-dimensional calculations. Annular-throat nozzles of the "plug" type also raised the question of the applicable local diameter to be used in the closed-form equation. Third, the closed-form equation provided only heat-transfer coefficients, whereas the increased precision required of rocket nozzle design made it desirable to know such boundary-layer parameters as the displacement and the momentum thicknesses. These thicknesses permit computation of nozzle performance corrections and provide nozzle-contour corrections for calculations of the free-stream flow. Thus, it appeared desirable to reformulate the turbulent boundary-layer heat-transfer equations in a form suitable to accommodate all of these new requirements, to eliminate compromises originally made to ease computational difficulties, and to program the result for digital-computer solution (14). At the same time, the analysis in Reference 9 was re-examined in the light of new information and altered where it seemed advisable.

The Blasius skin-friction formula employed in Reference 9 was replaced by Coles' correlation (15), which better fits the data at high Reynolds and Mach numbers. Momentum thickness was made the characteristic dimension in computing the skin-friction coefficient since this thickness is a more fundamental property of the boundary layer than the velocity thickness employed in Reference 9 , and is the dimension employed in Coles' correlation. Energy thickness with a correction for differing momentum thickness was made the characteristic dimension in computing Stanton number, rather than velocity thickness with a correction for temperature thickness. Mach number at the edge of the boundary layer was made an optional parameter to be prescribed in place of area ratio, facilitating application to nozzles of the bell and plug type. For convenience, axial distance, rather than distance along the wall, was made the position variable. Adiabatic recovery temperature, instead of stagn ation temperature, was made the driving potential in computing heat flux, improving accuracy at high Mach numbers, and provision was made for optionally employing enthalpy (rather than temperature) driving potential in cases in which chemical reaction or variable specific heat must be considered. The momentum and static-temperaturedistribution equations of Reference 9 were corrected to apply more accurately to unequal momentum and energy thickness. Finally, a simultaneous, iterative solution of the momentum and energy equations was formulated, rather than stopping at the first approximation as in Reference 9.

Unfortunately, the intervening years have shed no fundamental light on the most important postulate of Reference 9 , that the skin friction and heat flux at any point in a nozzle are the same as they would be on a flat plate at the same free-stream conditions and boundary-layer thickness. Furthermore, as discussed later, the question of a variable-properties correction for severely cooled boundary layers has become clouded rather than clarified. Although little more basic insight into these questions has 
been achieved, a considerable amount of supersonic-nozzle and rocket-thrust-chamber data has been obtained with which some degree of gross comparison can be made. The need for additional specific experimental results will become apparent from the comparisons. 


\section{ANALYSES}

\section{A. Integral Momentum and Energy Equation Solution}

The only method of approach amenable to analysis known to this author for the solution of the turbulent boundary-layer development and local heat transfer in rapidly accelerating flows is the simultaneous solution of the integral forms of the boundary-layer momentum and energy equations. As mentioned in the Introduction, References 8 and 9 describe some of the earliest attempts at such solutions. Others have been discussed in the literature but have differed only in minor detail or, in the interest of devising a simpler method, have necessarily involved considerably more arbitrary assumptions and heuristic arguments. It is beyond the scope of this Chapter to critically review and compare detailed results from these analyses since the differences in most cases are small compared with the differences between the predictions and experimental results. The purposes of this Chapter are served satisfactorily by the derivations adapted from Reference 14 and presented in this Section, which illustrate the essential features of the analyses. It is important to recall that one necessarily proceeds with analyses of the turbulent boundary layer primarily through plausible assumptions and intuitive arguments. Fortunately, errors and uncertainties introduced by approximations made in determining the development of the boundary layers are reduced considerably by the fact that the boundary-layer thicknesses enter into the heat-transfer coefficient to about the $1 / 4$ power. The most direct effect on the heat-transfer coefficient is encountered in the skin-friction coefficient and Stanton-number correlations adopted. Unfortunately, the correlation equations must be based on experiments, the results of which do not always agree.

The integral momentum and integral energy equation of the turbulent boundary layer are usually derived either (1) from integration of the Prandtl boundary-layer equations, with certain questionable assumptions made about the turbulent fluctuation correlation terms or (2) from the control-volume viewpoint, in which these turbulent fluctuation terms are ignored. The derivation presented here, although related to the second approach, differs by starting with the displacement, momentum, and energy boundarylayer thicknesses as basic definitions of the respective deficiencies in mass, momentum, and energy resulting from friction and heat transfer. This derivation is based on comparison of the real flow, with a hypothetical adiabatic potential flow extending all the way to the wall of a slightly different nozzle and having the same wall static-pressure distribution and total mass flux as the real flow. In the following treatment, the nomenclature employed for the real-flow and potential-flow nozzles will be introduced first, 
followed by the definitions of the displacement, momentum, and energy thicknesses, a discussion of the assumptions employed, the derivation of the integral momentum and energy equations, the presentation of the skin-friction and heat-transfer correlation equations adopted, and the derivation of relations for the boundary-layer shape parameters linking the various thicknesses.

\section{Nomenclature}

a. Real Flow. Figure 1 presents the nomenclature for the real nozzle flow. 'The stagnation conditions of the gas flowing through the nozzle are: temperature $T_{0}$, pressure $p_{0}$, specific heat $c_{p}$, specific heat ratio $\gamma$, Prandtl number $\operatorname{Pr}$, and viscosity $\mu_{0}$. At a given station, the distance along the nozzle axis is $z$, the distance along the wall is $x$, the radius of the wall from the axis is $r$, the wall temperature is $T_{w}$, the wall shear stress that retards the fluid motion is $\tau_{w}$, and the heat flux to the wall is $q_{w}$. At a distance $y$ from the wall, the time-mean values of the turbulently fluctuating density, stagnation temperature, and $x$-component of the velocity are $\vec{\rho}, \overline{t_{0}}$, and $\bar{u}$, respectively. The velocity $\bar{u}$ varies from zero at the wall to the free-stream value $U$ at distance $\delta$ from the wall; $\delta$ is the velocity thickness of the boundary layer. The stagnation temperature $\bar{t}_{0}$ varies from $T_{w}$ at the wall to the freestream value $T_{0}$ at distance $\Delta$ from the wall; $\Delta$ is the temperature thickness of the boundary layer.

There is a streamline of the flow, the $n$th streamline, which, for a finite distance upstream and downstream of station $z$, lies just beyond $\delta$ and $\Delta$. Thus, all boundary-layer effects are confined to a wall layer defined as containing the flow between the $n$th streamline and the wall. At station $z$, the $n$th streamline lies a distance $\delta_{r}^{\prime}$ from the wall, and the gas flowing at that point has density $\rho$, pressure $p$, Mach number $M$, static temperature $T$, and viscosity $\mu$. Although $\delta_{r}^{\prime}$ is greater than both $\delta$ and $\Delta$, the separation of these three points is assumed small enough to make the difference in free-stream properties between them negligible.

The fluxes of mass, momentum, and total enthalpy between the $n$th streamline and the wall for the real flow of Fig. 1 are $\dot{m}_{r}(\mathrm{lbm} / \mathrm{sec}), \dot{M}_{r}\left(\mathrm{ft} \mathrm{lbm} / \mathrm{sec}^{2}\right)$, and $\dot{H}_{r}$ (Btu/sec), respectively.

\section{b. Potential Flow. Figure 2 presents the nomenclature required for describing the} potential-flow nozzle, in which the conditions at the $n$th streamline conditions $U, \rho$, and $T_{0}$ extend all the way to the wall. The $n$th streamline in the potential-flow nozzle is at identically the same location with respect to the nozzle axis as in the real-flow nozzle, but the wall must, in general, be at a different

distance $\delta_{p}^{\prime}$ from the $n$th streamline in order to satisfy the requirement that the mass flux $\dot{m}_{p}$ between that 
streamline and the wall to remain equal to $m_{r}$. The momentum flux and enthalpy flux of $\dot{m}_{p}$ in the potentialflow nozzle are $\dot{M}_{p}$ and $\dot{H}_{p}$, respectively. Inder the assumption that boundary-layer effects are confined to a small distance from the wall, relative to $r$, the wall radius $r_{p}$ of the potential-flow nozzle is approximately equal to $r$. Results of the derivation of the integral momentum and energy equations for cases in which the boundary-layer thicknesses $\delta$ and $\Delta$ are not small with respect to $r$ are given in Appendix $C$.

\section{Definitions}

a. Deficiency Thicknesses. Under the assumption that $\delta_{p}^{\prime}$ is small compared with $r$, it is seen from Fig. 2 that the fluxes of mass, momentum, and enthalpy (referenced to the wall temperature $\left.\dot{T}_{w}\right)$ in the potential-flow nozzle are

$$
\begin{aligned}
& \dot{m}_{p}=2 \pi r \rho U \delta_{p}^{\prime} \\
& \dot{H}_{p}=2 \pi r \rho U^{2} \delta_{p}^{\prime} \\
& \dot{H}_{p}=2 \pi r \rho U c_{p}\left(T_{0}^{\prime}-T_{w}\right) \delta_{p}^{\prime}
\end{aligned}
$$

For $\delta_{r}^{\prime}$ also small compared with $r$, it is seen from Fig. 1 that the fluxes of mass, momentum, and enthalpy in the real-flow case are approximately

$$
\begin{aligned}
& \dot{m}_{r} \cong 2 \pi r \int_{0}^{\delta_{r}^{\prime}} \bar{\rho} \bar{u} d y \\
& \dot{M}_{r} \cong 2 \pi r \int_{0}^{\delta^{\prime}} \bar{\rho} \bar{u}^{2} d y \\
& \dot{H}_{r} \cong 2 \pi r \int_{0}^{\delta_{r}^{\prime}} \bar{\rho} \overline{u c}_{p}\left(\overline{t_{0}}-T_{w}\right) d y
\end{aligned}
$$

Equations (4), (5), and (6) are approximate in that a product of mean values is not, in general, equal to the mean value of the product; the cross-correlation terms must be considered. For example, the product $\bar{\rho} \bar{u}$ in Eq. (4) is not necessarily equal to the time-mean flow density $\rho$ u which would have to appear in Fq. (4) to make the equation exact. However, it can be argued that the cross-correlation terms 
substantially cancel out when the integration is performed over the boundary layer (16, p. 1090).

Since $\delta_{p}^{\prime}$ has been selected such that $\dot{m}_{r}=\dot{m}_{p}$, Fqs. (1) and (4) can be equated to yield the following expression for the difference in the wall positions between the two nozzles:

$$
\delta_{r}^{\prime}-\delta_{p}^{\prime}=\int_{0}^{\delta_{r}^{\prime}}\left(1-\frac{\bar{\rho} \bar{u}}{\rho U}\right) d y
$$

'The integral above is customarily defined as the displacement thickness $\delta$. Thus, the physical significance of the displacement thickness is that $\delta^{*}$ is the distance the wall must be moved inward or outward for adiabatic potential flow as compared with the position of the wall for a real flow having the same mass flux. That is, the physical definition of the displacement thickness is

$$
\delta^{*}=\delta_{r}^{\prime}-\delta_{p}^{\prime}
$$

while the integral definition is

$$
\delta^{*}=\int_{0}^{\delta_{r}^{\prime}}\left(1-\frac{\bar{\rho}-}{\rho U}\right) d y
$$

Because of the approximate nature of Fq. (7), (resulting from the approximation in Eq. 4), Eqs. (8) and (9) do not define exactly the same quantity. The question of which definition to adopt as fundamental will be discussed later.

Subtracting Fq. (5) from Eq. (2) yields, with the aid of Eq. (7), the deficiency of momentum flux in the real flow as compared with the potential flow:

$$
\dot{M}_{p}-\dot{M}_{r}=2 \pi r \rho U^{2} \int_{0}^{\delta_{r}^{\prime}} \frac{\bar{\rho} \bar{u}}{\rho U}\left(1-\frac{\bar{u}}{U}\right) d y
$$

The integral above is customarily defined as the momentum thickness $\theta$. Thus, the physical significance of the momentum thickness is that $\theta$ is the thickness of potential flow which has a momentum flux equal to that by which the momentum flux of the potential flow exceeds the momentum flux of the real flow for the same mass flux. Hence, the physical definition of the momentum thickness is 


$$
\dot{M}_{p}-\dot{M}_{r}=2 \pi r \rho U^{2} \rho
$$

and the integral definition is

$$
\theta=\int_{0}^{\delta_{r}^{\prime}} \frac{\bar{\rho} \bar{u}}{\rho U}\left(1-\frac{\bar{u}}{U}\right) d y
$$

Subtracting Fq. (6) from Eq. (3) yields, with the aid of Eq. (7), the deficiency of enthalpy flux in the real flow as compared with the potential flow:

$$
\dot{H}_{p}-\dot{H}_{r}=2 \pi r \rho U_{p}\left(T_{0}-T_{w}\right) \int_{0}^{\delta_{r}^{\prime}} \frac{\bar{\rho} \bar{u}}{\rho \bar{U}}\left(1-\frac{\overline{t_{0}}-T_{w}}{T_{0}-T_{w}}\right) d y
$$

The integral above is customarily defined as the energy thickness $\phi$. Thus, the physical significance of the energy thickness is that $\phi$ is the thicliness of potential flow which has an enthalpy flux equal to that by which the enthalpy flux of the potential flow exceeds the enthalpy flux of the real flow for the same mass flux. Hence, the physical definition of the energy thickness is

$$
\dot{H}_{p}-\dot{H}_{r}=2 \pi r \rho U c_{p}\left(T_{0}-T_{w}\right) \phi
$$

and the integral definition is

$$
\phi=\int_{0}^{\delta_{r}^{\prime}} \frac{\bar{\rho} \bar{u}}{\rho U}\left(1-\frac{\overline{t_{0}}-T_{w}}{T_{0}-T_{w}}\right) d y
$$

b. Coefficients. The skin-friction coefficient $C_{f}$ is defined as the ratio of the wall shear stress to the dynamic pressure of the flow at the edge of the boundary layers. Thus,

$$
C_{f}=\frac{2 \tau_{w}}{\rho U^{2}}
$$


'The Stanton number $C_{h}$ is defined as the ratio of the wall heat flux to the enthalpy flux of the flow at the edge of the boundary layers based on the difference between adiabatic and actual wall temperature. 'Thus,

$$
C_{h}=\frac{q_{w}}{\rho U_{p}\left(T_{a w} \cdots T_{w}\right)}
$$

The adiabatic wall temperature $T_{a w}$ is the wall temperature for sero heat flux and is related to Mach number by

$$
\frac{T_{a w}}{T_{0}}=\frac{1+\frac{\gamma-1}{2} R M^{2}}{1+\frac{\gamma-1}{2} M^{2}}
$$

where $R$ is the "adiabatic recovery factor."

\section{Assumptions}

The following assumptions are made in the analysis:

1. The flow is axisymmetric without tangential components of velocity and steady.

2. The boundary layer is confined to a distance from the wall which is small compared with the distance from the axis of symmetry.

3. The only forces acting on the gas are those due to pressure gradients and to skin friction at the wall.

4. The only changes in total enthalpy in the flow direction are those due to heat flux through the wall.

5. The flow immediately outside the boundary layer is reversible and adiabatic and parallel to the wall.

6. Static pressure is constant through the boundary layer perpendicular to the wall.

7. The gas is perfect; however, the restriction that specific heats be constant can be removed in computing the driving potential for heat flux.

8. The gas has a constant Prandtl number, a viscosity which varies as a power of 
the temperature, and a constant adiabatic recovery factor.

9. The skin-friction coefficient is the same as for constant-pressure constant-walltemperature flow on a flat plate at the same free-stream conditions, wall temperature, and momentum thickness.

10. The Stanton number is the same as for constant-pressure constant-wall-temperature flow on a flat plate at the same free-stream conditions, wall temperature, energy thickness, and momentum thickness.

11. The Stanton number for unequal momentum and energy thicknesses is that for equal thicknesses multiplied by $(\phi / \theta)^{n}$, where $n$ is a small "interaction exponent."

12. Heat transfer affects the skin-friction coefficient in one of two ways:

a. There is no effect, and $C_{f}$ is the same as for adiabatic flow, or

b. $C_{f}$ is the same as for adiabatic incompressible flow at a density and viscosity evaluated at the arithmetic mean between the actual wall temperature and the free-stream static temperature.

13. The Stanton number for equal momentum and energy thicknesses is related to the skin-friction coefficient by von Kármán's form of Reynolds' analogy.

14. Any chemical reactions in the boundary layer affect only the driving potential for heat flux.

15. The boundary-layer shape parameters $\theta / \delta, \Delta / \delta$, and $\delta^{*} / \theta$ are those for $1 / 7$-power profiles of velocity and of the difference between stagnation and wall temperature. Such profiles are typical of turbulent boundary layers on flat plates.

16. Heat transfer by thermal radiation is negligible compared with convection.

17. There is no significant net mass transfer from wall to gas or gas to wall.

Assumptions 1, 2, 3, and 4 define the situation to which the analysis applies. Assumption 3 excludes, for example, magnetohydrodynamic forces, and Assumption 4 excludes combustion effects (except for a possible direct effect on heat flux as allowed by Assumption 14). Assumptions 1 and 2 have already been employed in defining $\delta^{*}, \theta$, and $\phi$. Assumptions 5 and 6 are good approximations if the flow has no strong shocks. Assumptions 7 and 8 introduce little error for most gases.

Assumptions 9 and 10 are the ones which most affect the results, and they are also the most uncertain. That skin friction and heat flux have the flat-plate dependence on local conditions is certainly valid asymptotically for gradual nozzle contours $\left(d r / d z \rightarrow 0\right.$ and $\left.d T^{\prime} / d z \rightarrow 0\right)$, but the extent of departure 
in practical nozzles remains unexplored by experiment except for the limited data of Reference 17 which tend to support the present assumption. In the absence of a correlation of heat transfer obtained with large differences between momentum and energy thicknesses, Assumption 11 was selected based upon intuitive reasoning and was found to agree with a small sample of data to be discussed.

Assumption 12 has two options, either of which can be selected. Assumption 12a is based on recent experiments $(18,19,20,21)$, which as discussed in Section II D, showed no measurable effect of heat transfer on skin-friction coefficient or Stanton number for cooled boundary layers compared with values for adiabatic walls. Assumption $12 \mathrm{~b}$ is the widely used procedure of evaluating properties at some reference temperature. The one selected being the arithmetic mean between the wall and the static temperature at the edge of the boundary layer, gives only slightly different values of $C_{f}$ from those determined by the reference-temperature method (22) out to Mach numbers of interest for most nozzle flows. However, the background of data on which the "film" or "reference" methods were established for the cooled turbulent case is sketchy, consisting mainly of data with negligible temperature differences (i.e., $T_{w} / T_{0}$ only slightly less than unity) on average data over long pipe lengths in which uncertain axial property variations clouded the picture considerably, and data which generally scattered to the same extent as the magnitude of the variable properties corrections. These data, as well as newer data which conflict with the reference method, are discussed in Section II D. Thus, one is faced with the quandary of having doubt thrown upon the "accepted" variable properties correction by the new data and yet not being fully convinced that there should be no correction on the basis of the limited data cited. For this reason, the analysis presented here retains the option of either treatment of the variable properties question.

Assumption 13 is well substantiated by flat-plate and pipe-flow experiments, as will be shown later. Assumption 14 represents the computationally convenient viewpoint that the effect of chemical recombination can be accounted for by employing enthalpy (rather than temperature) driving potential, leaving the heat-transfer coefficient unaltered. Assumption 15 agrees roughly with observed velocity and temperature profiles on flat plates and wind-tunnel nozzles. The only effect of $\Lambda$ ssumption 15 on the other parameters computed, however, is through the ratio $\delta^{*} / \theta$ which is relatively insensitive to the profiles assumed, and which, in turn, has only a secondary effect on momentum thickness and skin friction, and little or no effect on energy thickness and heat flux. Assumptions 16 and 17 are, of course, only statements of the limit to the scope of the analysis. 


\section{Integral Equations}

The usual approach to the derivation of the integral momentum and energy equations for a turbulent boundary layer $(9,16)$ is to start with the boundary-layer differential equations and introduce an approximation by eliminating the fluctuating cross-correlation terms through arguments that they substantially cancel out when integrated across the boundary layer (16, p. 1090). Through this process, one arrives at integrals of time-averaged variables such as

$$
\int_{0}^{\delta_{r}^{\prime}}\left(1-\frac{\bar{\rho} \bar{u}}{\rho U}\right) d y
$$

which are then defined as exactly equal to new variables $\delta^{*}, 0$, and $\phi$. In this case, the definitions given by Eqs. (9), (12), and (15) are considered fundamental. However, the variables $\delta^{*}, \theta$, and $\phi$ are then related only approximately to the physical mass, momentum, and energy defects, and the resulting momentum and energy equations become approximations of uncertain accuracy when written in terms of these integrally defined variables.

An alternate derivation of the integral momentum and energy equations, which will be presented here, adopts at the outset the physical definitions of $\delta^{*}, \theta$, and $\phi$ given, respectively, by Eqs. (8), (11), and (14). It will be seen that this derivation leads directly to the integral momentum and energy equations without further approximation and without consideration of the internal structure of the boundary layer. The resulting equations are identical in appearance with those derived from the differential equations, differing only in the definitions associated with $\delta^{*}, \theta$, and $\phi$. However, the uncertainty in the integral expressions for $\delta^{*}, \theta$, and $\phi$ due to the turbulent fluctuation terms may still affect the results, to a minor extent, through the use of the integral expressions in the shape parameters introduced later, and in evaluating Reynolds numbers in most of the available skin-friction data.

a. Momentum Equation. For the potential flow along the wall, $\dot{m}_{p}$ the streamwise gradient of momentum flux $\dot{M}_{p}$ is, by Assumption 3, balanced only by the pressure gradient acting over the flow area $2 \pi r \delta_{p}^{\prime}$, where the latter, from the physical definition of the displacement thickness, Eq. (8), is equal to $2 \pi r\left(\delta_{r}^{\prime}--\delta^{*}\right)$. Thus, employing the physical definition of the momentum thickness from Fi. (11), the momentum-flux gradient is 


$$
\frac{d}{d x}\left(\dot{M}_{r}+2 \pi r \rho U^{2} \theta\right) \cdots-2 \pi r\left(\delta_{r}^{\prime} \delta^{*}\right) \frac{d p}{d x}
$$

Fior the real wall flow $\dot{m}_{r}$, the streamwise gradient of momentum flux $\dot{M}_{r}$ is balanced by both the wall shear force and the pressure gradient acting over the area $2 \pi r \delta_{r}^{\prime}$. 'I'hus,

$$
\frac{d}{d x} \dot{M}_{r}=-2 \pi r \tau_{w}-2 \pi r \delta_{r}^{\prime} \frac{d p}{d x}
$$

Subtracting Fq. (20) from Fq. (19), and noting that by Assumptions 5 and $7 d p / d x=-\rho U d U / d x$, the following relation is obtained:

$$
\frac{d}{d x}\left(r \rho U^{2} \rho\right)=r \tau_{w}, \cdots r \rho U \delta^{*} \frac{d U}{d x}
$$

Fquation (21) is the integral momentum equation for thin axisymmetric boundary layers. It can be put in a more convenient form by differentiating, introducing the definition of the skin-friction coefficient, Fq. (16), and rearranging to give

$$
\frac{d \theta}{d x}=\frac{C_{f}}{2}-\theta\left[\frac{1+\frac{\delta^{*}}{O}}{U} \frac{d U}{d x}+\frac{1}{\rho U} \frac{d(\rho U)}{d x}+\frac{1}{r d x}\right]
$$

Under Assumptions 5 and 7 , the expressions involving $\rho$ and $U$ can be written in terms of the Mach number $M$, as follows:

$$
\begin{aligned}
& \frac{1}{U} \frac{d U}{d x}=\frac{1}{M\left(1+\frac{\gamma-1}{2} M^{2}\right)} \frac{d M}{d x} \\
& \frac{1}{\rho U} \frac{d(\rho U)}{d x}=\frac{1-M^{2}}{M\left(1+\frac{\gamma-1}{2} M^{2}\right)} d x \\
& d x
\end{aligned}
$$


Substituting these expressions into Fq. (22) and transforming the independent variable to $z$ by noting that $d x / d z=\left[1+(d r / d z)^{2}\right]^{1 / 2}$, the final form of the integral momentum equation is obtained:

$$
\frac{d \theta}{d z}=\frac{C_{f}}{2}\left[1+\left(\frac{d r}{d z}\right)^{2}\right]^{1 / 2}-\theta\left[\frac{2-M^{2}+\frac{\delta^{*}}{\theta}}{M\left(1+\frac{\gamma-1}{2} M^{2}\right)} \frac{d M}{d z}+\frac{1}{r} \frac{d r}{d z}\right]
$$

b. Energy Equation. For the wall flow $\dot{m}_{p}$ without heat transfer, the enthalpy flux, by Assumption 4, remains constant. Thus, employing the physical definition of the energy thickness from Eq. (14), the streamwise gradient of the enthalpy flux $\dot{H}_{p}$ is

$$
\frac{d}{d x}\left[\dot{H}_{r}+2 \pi r \rho U c_{p}\left(T_{0}-T_{w}\right) \phi\right]=0
$$

For the real wall flow $\dot{m}_{r}$, the streamwise gradient of the en thalpy flux $\dot{I}_{r}$ is exactly equal to minus the rate at which energy is transferred to the wall. Thus,

$$
\frac{d}{d x} \dot{H}_{r}=-2 \pi r q_{w}
$$

Subtracting Eq. (27) from Fiq. (26) yields

$$
\frac{d}{d x}\left[r \rho U c_{p}\left(T_{0}-T_{w}\right) \phi\right]=r q_{w}
$$

Fquation (28) is the integral energy equation for thin axisymmetric boundary layers. It can be put in a more convenient form by differentiating, introducing the definition of the Stanton number (Eq. 17), and rearranging to give

$$
\frac{d \phi}{d x}=C_{h}\left(\frac{T_{a w}-T_{w}}{T_{0}-T_{u,}}\right)-\phi\left[\begin{array}{c}
1 \\
\hdashline U \frac{d(\rho U)}{d x}+\frac{1}{r} \frac{1}{d x}-\frac{1}{T_{0}-T_{w}} d x
\end{array}\right]
$$


Substituting Eqs. (23) and (24) and transforming the independent variable to $z$ yields the final form of the integral energy equation

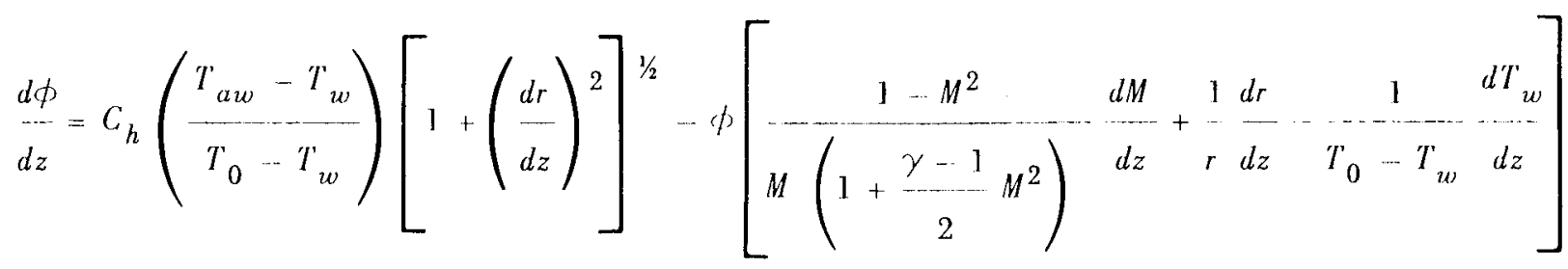

Note that the effect of variable surface temperature is accounted for in the development of the energy thickness boundary layer. This is the general relationship upon which the usual variable surfacetemperature correction to heat transfer on a flat plate is based.

\section{Skin-Friction Coefficient}

a. Diabatic Skin-Friction Coefficient. In accordance with Assumption 9, the skinfriction coefficient in a nozzle is taken to be the same as that on a flat plate at the same conditions at the edge of the boundary layers $\rho, U, \mu, T_{0}$, and $M$, the same wall temperature $T_{w}$, and the same momentum thickness $\theta$. Unfortunately, even this drastic assumption does not permit a completely reliable evaluation of $C_{f}$, since only the adiabatic skin-friction coefficient $C_{f_{a}}$, obtained when $T_{u}=T_{a w}$, is known accurately. The relationship between $C_{f}$ and $C_{f_{a}}$ for severely cooled turbulent boundary layers, when gas properties vary greatly between the free stream and the wall, is sufficiently uncertain that both relationships discussed earlier are included in the analysis as alternatives.

The first relationship, Assumption 12a, is that of computing the value of $C_{f}$ by assuming it to be exactly equal to that for an adiabatic wall, i.e.,

$$
\frac{C_{f}}{C_{f_{a}}}=1
$$

The correlation of adiabatic turbulent boundary-layer skin-friction coefficients developed by Coles in Reference 15 was found to correlate accurately the trends and magnitudes of nearly all of the reliably measured data from high-speed flow over flat plates thus far reported in the literature. In particular, it fits the data at high Reynolds and Mach numbers better than previously used correlations. Consequently, 
in updating the analysis of Reference 9 the Coles correlation (the details of which are presented in Appendix $\Lambda$ ) was adopted in Reference 14 and was used in obtaining the new boundary-layer calculation results presented in this Chapter. Unfortunately, since the Coles correlation is not explicit in the momentum thickness Reynolds number $R_{\theta}$, the important functional relationships of the analysis become difficult to recognize. Therefore, in order to illustrate the essential features of the problem in the clearest manner, the Blasius equation, coupled with variable properties corrections, will be used in the remainder of this Section. (Comparing the Blasius cquation with the tabulated values of low-speed skinfriction coefficients $\bar{C}_{f}$ used in the Coles correlation [T'able A-I, Fig. 3], the maximum deviation is $5 \%$ between $R_{\theta}$ of 400 and $R_{\theta}$ of 15,000 .) The Blasius equation, expressed in terms of $R_{\theta}$, is

$$
\bar{C}_{f}=\frac{0.0256}{\left(R_{\theta}\right)^{1 / 4}}
$$

where $\bar{C}_{f}$ is the low-speed value of skin friction, $R_{\theta}$ equals $\rho U \theta / \mu$, and $\rho$ and $\mu$ are properties evaluated at the local static temperature $T$ at the edge of the boundary layers. The relation between $C_{f_{a}}$ and $\bar{C}_{f}$ in the Coles correlation, derived in Appendix A, is

$$
\frac{C_{f_{a}}}{\bar{C}_{f}}=\frac{1}{\left(\frac{T_{a w}}{T}\right)\left(\frac{T_{s}}{T_{a w}}\right)^{m}}
$$

where $T_{s}$ is a sublayer temperature specified in Eq. (A-3) of Appendix A, and $m$ is the exponent of the viscosity relationship adopted, $\mu \sim T^{m}$. For our purposes, Eq. (33) can be approximated by

$$
\frac{C_{f_{a}}}{\bar{C}_{f}}=\frac{1}{\left(\frac{T_{a w}}{T}\right)^{0.6}}
$$

with less than about $10 \%$ error over the range of $R_{\theta}$ and $M$ of interest for $m=0.6$. Combining Eqs. (31), (32), and (34), 


$$
C_{f}=\frac{0.0256}{\left(R_{\theta}\right)^{1 / 4}}\left(\begin{array}{c}
T_{a w} \\
T
\end{array}\right)^{-0.6}
$$

'The second relationship, Assumption $12 \mathrm{~b}$, is that of accounting for the effect that property variation may have on skin friction, either as a result of compressibility, or of $T_{w} \neq T_{a w}$, or both, by evaluating the properties $\rho$ and $\mu$ at a temperature which is the arithmetic mean between $T$ and $T^{\prime}$. This is the same relationship employed in Reference 9 and elsewhere. The reasoning behind such a correction and a discussion of its validity are presented in Section II D.

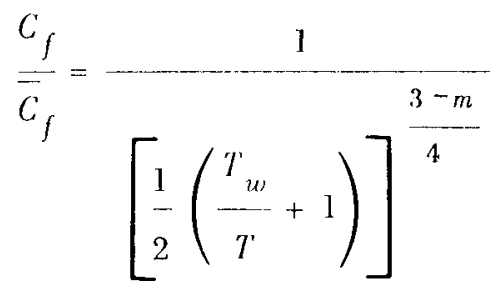

Or, again making use of Fq. (32) and assuming $m=0.6$,

$$
C_{f}=\frac{0.0256}{\left(R_{\theta}\right)^{1 / 4}}\left[\frac{1}{2}\left(\frac{T_{w}}{T}+1\right)\right]^{-0.6}
$$

\section{Stanton Number}

For flow with substantial pressure gradients, it is no longer possible to derive an appropriate analogy between the Stanton number $C_{h}$ and $C_{f} / 2$ in a straightforward manner because the similarity between the momentum and energy equations is destroyed by the presence of the pressuregradient terms. Nevertheless, in order to proceed, it was found necessary to adopt some form of Reynolds analogy, modified according to arguments in this Section. By Assumption 10, the Stanton number $C_{h}$ in a nozzle is taken to be the same as that on a flat plate at the same free-stream conditions $\rho, U, \mu, T_{0}, M$, the same wall temperature $T_{w}$, and the same local energy and momentum thicknesses $\phi$ and $\theta$.

'I'he most appropriate Prandtl-number correction to the Reynolds analogy is believed to be the von Kármán form (2.3, p. 225), which was derived by consideration of the respective thermal resistances in a laminar sublayer, a buffer layer, and a turbulent outer region. 


$$
C_{h}=\frac{\frac{C_{f}}{2}}{1-5\left(\frac{C_{f}}{2}\right)^{1 / 2}\left[1-P r+\ln \left(\frac{6}{5 P r+1}\right)\right]}
$$

A Prandtl-number correction of this form has been utilized in all of the new boundary-layer calculations from which results are presented in this Chapter. Again, however, as in the previous Section, the purposes of illustration of the most significant relationships of the problem can be better served by replacing Eq. (38) with the simpler but less widely valid Colburn form of Reynolds analogy.

$$
C_{h}=\frac{\frac{C_{f}}{2}}{p_{r}{ }^{2 / 3}}
$$

It is important to note at this point that relationships such as Eqs. (38) and (39) have been established by experimental correlation and analysis of flows in which the ratio of energy thickness to momentum thickness, $\phi / \theta$, is essentially constant in the streamwise direction at a value close to unity, being dependent only upon a small fractional power of the Prandtl number. In the nozzle flow situation, analyses of the type presented in References 8 and 9 have predicted that the ratio $\phi / \theta$ may increase to values as high as 5 in the throat region because of the differences in the integral momentum and energy equations resulting from the combined presence of the term

$$
-\theta\left[\frac{1+\frac{\delta^{*}}{\theta}}{M\left(1+\frac{\gamma-1}{2} M^{2}\right)} \frac{d M}{d z}\right]
$$

in the momentum equation and the term

$$
+\phi\left[\frac{1}{\left(T_{0}-T_{w}\right)} \frac{d T_{w}}{d z}\right]
$$


in the energy equation. Thus, it was apparent in the analyses of Ref. 8 and 9 that some account must be taken of $\phi / 0 \neq 1$. In those references, the Reynolds analogy was modified by multiplication by a factor $(\Delta / \delta)^{-1 / 7}$, which is fairly close to $(\phi / \theta)^{-1 / 7}$ for most conditions of interest. The exponent was arrived at by intuitive arguments linked to the power-relation velocity and stagnation temperature distributions assumed to exist in the boundary layer. Again following such reasoning in this development but broadening it slightly by allowing the exponent to be a parameter to be chosen, the result is

$$
C_{h}=\frac{\frac{C_{f}}{2}\left(\frac{\phi}{\theta}\right)^{n-1 / 4}}{\operatorname{Pr}^{2 / 3}}
$$

Substituting into Eq. (40) the expression for $C_{f} / 2$ from Eqs. (35) and (37), which can be made equivalent in form by expression in terms of a temperature $T_{\text {ref }}$, [ where in Eq. (35) $T_{\text {ref }}=T_{a w}$, and in Eq. (37) $\left.T_{\text {ref }}=\left(T_{w}+T\right) / 2\right]$, the result is

$$
C_{h}=\frac{0.0128\left(\frac{T_{\text {ref }}}{T}\right)^{-0.6}}{\operatorname{Pr}^{2 / 3}} \frac{\left(\frac{\phi}{\theta}\right)^{n-1 / 4}}{\left(\frac{\rho U \theta}{\mu}\right)^{1 / 4}}
$$

or

$$
C_{h}=\frac{0.0128\left(\frac{T_{\mathrm{ref}}}{T}\right)^{-0.6}}{\operatorname{Pr}^{2 / 3}} \frac{1}{\left(\frac{\rho U}{\mu}\right)^{1 / 4} \rho^{n} \phi^{1 / 4-n}}
$$

Noting that $C_{h}$ increases sharply as the down stream distance approaches zero from the start of a thermal boundary layer (i.e., location of start of heating or cooling downstream of the flow origin), it might be argued that the upper limit for $n$ must be $1 / 4$. On the other hand, it might be argued intuitively that $n$ 
should probably not fall below zero since a growing momentum thickness would be predicted to increase $C_{h}$ contrary to the usual behavior. (The value of $n$ corresponding to the $(\Delta / \delta)^{-1 / 7}$ factor of References 8 and 9 is $3 / 28$, or about 0.1 .)

One might consider rearranging Eq. (42) to

$$
C_{h}=\frac{0.0128\left(\frac{T_{\mathrm{ref}}}{T}\right)^{-0.6}}{\operatorname{pr}_{r}^{2 / 3}} \frac{\left(\frac{\phi}{\theta}\right)^{n}}{\left(\frac{\rho U \phi}{\mu}\right)^{1 / 4}}
$$

This form suggests that a Reynolds-number-like parameter $R_{\phi}=\rho U \phi / \mu$ is perhaps equally relevant to the correlation of $C_{h}$ as $R_{\theta}$ is to the correlation of $C_{f}$. Such a suggestion has been made previously by Seban and Chan (24) and Kutateladze and Leontev (25) and used to advantage in their analyses. In addition, an equation of the form of Eq. (43), derived in Appendix A (Eq. A-8) using the Coles and von Kármán relations, has been utilized to correlate one set of data for a flat-plate flow (19) and another for tube-entrance flow (20). The $R_{x}$ values with which the data of Reference 19 were correlated were multiplied by $\phi / x$, where $\phi / x$ was computed from the low-speed, constant-surface-temperature, flat-plate energy equation, $d \phi / d x=C_{h}$. Since these data were obtained with $T_{w} / T_{0}$ between 1 and 1.05 , i.e., with a low temperature difference across the boundary layer, the variable properties factor differed only negligibly from unity irrespective of the choice of $T_{\text {ref }}$. In Reference 20 , values have been presented of the heat flux in successive separately cooled sections downstream of the abrupt start of cooling of a fully developed adiabatic pipe flow. These values have been used to obtain $\phi$ and the values of $\phi$ differentiated to determine local $C_{h}$. In these tests, with both air and $\mathrm{CO}_{2}$, severe wall cooling was employed, yielding values of $T_{w} / T_{0}$ between 0.3 and 0.7 . In computing $R_{\phi}, \rho$, and $\mu$ were evaluated at the local free-stream temperature, which is equivalent to assuming $T_{\text {ref }}=T_{a w}$ for this low-speed flow. The data from these two references plotted as $C_{h}(\phi / \theta)^{-0.1}$ versus $R_{\phi}$ are compared with $\mathrm{Eq}$. (A-8) in Fig. 4 and found to agree quite satisfactorily. Such agreement between the Reference 19 data and Eq. (A-8) should, of course, be expected, since both $T_{w} / T_{0}$ and $\phi / \theta$ are near unity. It is significant, however, that most of the data of Reference 20 also agree with the Equation to within $\pm 10 \%$ even though they were obtained with a wide range of values of both $T_{w} / T_{0}$ and $\phi / \theta$. A limited sample of data such as this 
cannot, of course, be used to make a sensitive determination of the most appropriate value of $n$, the interaction parameter. Consequently, a value of $n$ of 0.1 was arbitrarily selected and found to improve the correlation somewhat compared with no correction at all (i.e., $n$ of 0 ). The data of Reference 20 also suggest that values of $C_{h}$ are insensitive to variations of $T_{w}^{\prime} / T_{0}^{\prime}$ for cooling since, by taking $T_{\text {ref }}=T_{a w}=T$, no correction for property variation has been made and no systematic deviation of the data with $T_{w} / T_{0}$ is noted. Agreement with this trend was also exhibited by data obtained from several other experimental investigations discussed in Section II D.

\section{Solution of Integral Equations}

With the specification of $C_{h}$ by Eq. (43) (or its more accurate counterpart, Eq. A-8) and $C_{f}$ by Eqs. (35) or (37) (or their more accurate counterparts, Eqs. 33, A-4, A-5, and Table A-1), only the local Mach number at the edge of the boundary layer and the boundary-layer shape parameter $\delta^{*} / \theta$ need be determined in order to proceed with the solution of the integral momentum and integral energy equations for $O$ and $\phi$, respectively. The local $M$ distribution is, of course, a function of the nozzle configuration and may be taken as that for one-dimensional reversible adiabatic flow, or that for twodimensional flows resulting from method-of-characteristics solutions where necessary (see Section III D). In order to compute the value of $\delta^{*} / \theta$ as well as some of the auxiliary shape parameters $\theta / \delta, \phi / \Delta$, and $\Delta / \delta$ to which $\delta^{*} / \theta$ is related, it is necessary to specify some velocity and stagnation-temperature distribution through the boundary layer. For this purpose, $1 / 7$-power distributions of $\bar{u}$ in terms of $y / \delta$ and $\bar{t}_{0}-T_{w}$ in terms of $y / \Delta$ have been adopted. 'These distributions obviously give grossly inaccurate values of $\bar{u}$ and $\left(\bar{t}_{0}-T_{w}\right)$ and their derivatives very near the wall. Fortunately, these distributions are utilized only in integration across the boundary layer in computing the shape parameters. Hence, the errors near the wall have negligible over-all effect. In fact, the shape parameters are quite insensitive to the arbitrarily specified exponent over a range from about $1 / 5$ to $1 / 9$ but are quite sensitive to the local value of $M$ and $T_{w} / T_{0}$ for which the value of the shape parameter is being computed. Thus, the use of low-speed adiabatic values of the shape parameters rather than the correct values obtained from distributions and integrals as presented in Appendix B can lead to significant errors near the nozzle throat. It is evident from the shape-parameter integrals that the shape parameters depend upon the ratio $\phi / \theta$ and that, consequently, the integral momentum and energy equations $(25,30)$ must be solved iteratively, except when the equations have been uncoupled by assuming $n=0$, and the expression for $C_{h}$ given by $\mathrm{Fq.} \mathrm{(43)} \mathrm{is} \mathrm{utilized.} \mathrm{Hence,} \mathrm{except} \mathrm{for} \mathrm{this} \mathrm{special} \mathrm{case,} \mathrm{it} \mathrm{becomes} \mathrm{almost} \mathrm{essential} \mathrm{to}$ program the equations for a digital computer since no simple analytic solution can be obtained without 
considerable compromise. Such a program is described in detail in Reference 14. In Reference 9 (written before the availability of a high-speed digital computer), the solution of the integral momentum and energy equations was not obtained by iteration but was terminated with a "first approximation," which was made by assuming a reasonable value of $\delta / \Delta$ to be a constant 1.0 only for the purpose of evaluating the boundary-layer shape parameters. Although it was argued at the time that such an assumption would have little effect, it was found that it does have a large effect on $\delta^{*} / \theta$ and even a significant effect on $h_{g}$, as will be demonstrated. In particular, in the throat region of a highly cooled $\left(T_{w} / T_{0}<<1\right)$ nozzle of typical rocket-nozzle shape, $\phi / \theta$, and hence $\Delta / \delta$, may approach values as high as 5 and, as a consequence, $\delta^{*} / \theta$ becomes negative. This was predicted and readily explained by Reshotko in his discussion of Reference 9. Because of the extreme cooling, the temperature falls faster than does the velocity across the boundary layer. The result is that the local mass flux per unit area in the cooled boundary layer exceeds that in the free stream, giving rise to the negative displacement thickness.

Finally, the local heat flux $q_{w}$ is computed from the defining equation for $C_{h}$, (Eq. 17), where $C_{h}$ has been determined as a function of $\theta$ and $\phi$ from an equation such as Eq. (43) (or its more accurate counterpart, Fq. A-8). To make this calculation, choices must be exercised as to (a) the method of properties evaluation - either $\rho$ and $\mu$ at film temperature or $C_{f}$ for an adiabatic wall by way of the Coles correlation and (b) the value of $n$, the interaction parameter, between 0 and $1 / 4$.

For the special case in which $n$ is assumed to be equal to zero and Eq. (43) is utilized to specify the functional relationship for $C_{h}$, the integral energy equation can be analytically solved separately from the integral momentum equation. The form of Eq. (30) under these conditions is

$$
\frac{d \phi^{5 / 4}}{d z}+\phi^{5 / 4} P(z)=Q(z)
$$

where

$$
P(z)=\frac{5}{4}\left[\frac{d}{d z} \ln \frac{T_{w}-T_{0}}{r}\right]
$$


where it has been assumed $\rho U_{r}^{2}=$ constant, and

$$
Q(z)=\frac{\frac{5}{4}(0.0128)\left(\frac{T_{\text {ref }}}{T}\right)^{-0.6}}{\operatorname{Pr}^{2 / 3}\left(\frac{\rho U}{\mu}\right)^{1 / 4}\left(\frac{T_{a w}-T_{w}}{T_{0}-T_{w}}\right)}\left[1+\left(\frac{d r}{d z}\right)^{2}\right]^{1 / 2}
$$

The solution of Eq. (44) is

$\phi^{5 / 4}=\phi_{o}^{5 / 4}\left(\frac{T_{0}-T_{u, z}}{T_{0}-T_{w, o}{ }_{o} r_{z}}\right)^{-5 / 4}+\left(\frac{T_{0}-T_{w, z}}{r_{z}}\right)^{-5 / 4} \int_{0}^{z}\left(\frac{T_{0}-T_{w, z}}{r_{z}}\right)^{5 / 4} Q(z) d z$

It must be remembered, however, that Eq. (45) is based upon three approximations: (1) the Blasius lowspeed skin-friction coefficient, (2) the Colburn form of Reynolds analogy for Prandtl-number correction, and (3) the approximation that $\left(T_{a w} / T\right)^{0.6} \approx\left(T_{a w} / T\right)\left(T_{s} / T_{a w}\right)^{0.6}$ for $m=0.6$ when basing the calculation on $C_{f}$ for adiabatic wall. For a film-temperature properties calculation, only the first two approximations pertain. The degree of approximation compared with what are believed to be the most valid values of $Q(z)$ varies with both $R_{\phi}$ and $M$. Under an extreme set of conditions, $M=4, R_{\theta}=R_{\phi} \approx 10^{4}$, the deviation of the value $Q(z)$ resulting from these approximations is about $+35 \%$. At lower $M$ and $R_{\phi}$, the deviation is considerably less. If, however, $Q(z)$ were taken to be $35 \%$ high throughout the nozzle, this would have about a $-6 \%$ effect upon $C_{h}$ through the value of $\phi^{1 / 4}$ for the worst case, when $\phi_{o}$ is zero. Since $C_{h} \sim Q(z) \phi^{-1 / 4}$, the net error in $C_{h}$ for the case cited is about $27 \%$, which can be reduced by refining the approximations listed above.

\section{Sample Results}

In order to demonstrate the qualitative behavior of the boundary layer and heat transfer in a typical small nozzle operating under typical rocket conditions, a sample calculation has been made for the nozzle depicted in Fig. 5 for the conditions listed. The results obtained by selecting the same nozzle configuration and operating conditions of a similar calculation in Reference 9 also show the effect of making an "iterative simultaneous" solution of the integral momentum and energy equations (as outlined in Section II A, 1-7) rather than the "first-approximation" solution as in Reference 9. The 
same three pairs of inlet boundary layer thicknesses $\delta$ and $\Lambda$ have been selected. In Fig. 6 , the values of $\delta$ presented show that a thick inlet boundary layer shrinks rapidly as a result of the subsonic acceleration, reaching a minimum just ahead of the throat, whereas a thin inlet boundary layer initially grows rapidly and then, too, shrinks to about the same value as the flow approaches the throat. It is also evident that the iterative simultaneous solution predicts considerably thicker boundary layers throughout the nozzle than first-approximation solution, although the qualitative behavior is essentially unchanged. In Fig. 7, a similar comparison of values of $\Delta$ is presented. Here, the changes between the first-approximation and the iterative simultaneous solution are somewhat smaller, as one might suspect, since the most significant change that occurs is the value of $\delta^{*} / \theta$ becoming negative in the momentum equation. 'The behavior of $\delta^{*}$ as determined by the iterative simultaneous solution is illustrated in Fig. 8 . As stated and explained in Section II $\Lambda-7$, the values of $\delta^{*}$ are negative over most of the length of the nozzle. Finally, the local $h_{g}$ distributions for the three assumed entrance conditions are presented in Fig. 9 and compared with those of the first-approximation solution. The thicker boundary layers $\delta$ determined for the iterative simultaneous solution effect approximately a $15 \%$ reduction in the maximum values of $h_{g}$. It should be noted that in order to permit comparison with the $h_{g}$ distribution of Reference 9, the film-temperature property evaluation option (Assumption 12b) was utilized. It is seen that the initial value of $\delta$ or $\theta$ has little effect on the $h_{g}$ distribution over most of the nozzle when the initial temperature boundary-layer thickness is small.

\section{B. Closed-Form Approximation}

From results such as those discussed in Section A, it was evident that the variation of local mass flow rate per unit area at the edge of the boundary layer is still the dominant variable affecting the heat-transfer distribution even in an accelerating flow. This suggested that by selecting some linear dimensional variable that varied in even a rough approximation to the variation of the boundary layer, a closed-form Nusselt - Reynolds type correlation equation could be found that might approximate the the results of the boundary-layer analysis reasonably well. Such an equation was developed in Reference 1.3 by selecting the local diameter at the station of the nozzle of interest as the linear dimension. This became an obvious choice when boundary-layer distribution results such as those shown in Fig. 6 were obtained. Only near the nozzle entrance for an initially thin boundary layer did this selection appear to be qualitatively inappropriate. 
Starting with the assumption that the local heat-transfer coefficient is principally dependent on local mass flow rate per unit area,

$$
h_{g} \sim(\rho U)^{k}
$$

The exponent as established by the results of the boundary-layer solutions is 0.8 because of the direct influence of the skin-friction correlation adopted. If Eq. (46) is nondimensionalized and is multiplied by a function of $\operatorname{Pr}$ as suggested by Eq. (38), one obtains the familiar Nusselt - Reynolds type equation

$$
N u=C(R e)_{D}^{0.8} \operatorname{Pr}_{r}^{0.4}
$$

where Reynolds number is based on the local diameter $D$, which was assumed to be the characterizing linear dimension. For a range of Prandtl numbers near unity it can be shown that the von Kármán Prandtl correction (Eq. 38) can be reasonably approximated by $P r^{0.4}$ for low values of $R_{\theta}$. Thus, the closed-form approximation is complete except for an arbitrary constant $C$. For the purposes of this equation the value of $C^{\prime}$ might be selected so as to force exact agreement of the closed-form equation and results of a boundary-layer solution at one particular point in the nozzle for a particular set of operating conditions. In Reference $13, C$ was evaluated to be 0.026 by forcing agreement at the throat with first-approximation results for the conditions listed and the nozzle configuration of Fig. 5. For other nozzle configurations and conditions, this constant would vary but not very drastically, especially if a factor $\left(D_{*} / r_{c}\right)^{0.1}$, as suggested by nozzle similarity considerations of Reference 9 , were multiplied into the equation.

The possible influence of variable properties considerations are readily demonstrated by the closed-form approximation. If it is assumed that both $c_{p}$ and $P_{r}$ are constant over the boundary layer (as they are to a close approximation for a wide temperature interval), the properties whose variations must be accounted for are only $\mu$ and $\rho$. If these are evaluated at some reference temperature to be determined, then Eq. (47) can be used to determine $h_{g}$, as follows:

$$
h_{g}=\frac{C}{D^{0.2}}\left(\frac{D_{*}}{{ }{ }_{c}}\right)^{0.1}\left(\frac{\mu_{0}^{0.2} c_{p}}{P_{r}^{0.6}}\right)^{(\rho U)^{0.8}}\left[\left(\frac{\rho_{\mathrm{ref}}}{\rho}\right)^{0.8}\left(\frac{\mu_{\mathrm{ref}}}{\mu_{0}}\right)^{0.2}\right]
$$


If, for the moment, the reference condition is assumed to be at a temperature halfway between the wall temperature and free-stream static temperature, the variable properties factor can readily be expressed in terms of $T_{u /} / T_{0}$ and $M$.

$\sigma=\left[\left(\frac{\rho_{\text {ref }}}{\rho}\right)^{0.8}\left(\frac{\mu_{\mathrm{ref}}}{\mu_{0}}\right)^{0.2}\right]=\frac{1}{\left[\frac{1}{2}\left(\frac{T_{w}}{T_{0}}\right)\left(1+\frac{\gamma-1}{2} M^{2}\right)+\frac{1}{2}\right]^{0.8-m / 5}\left[1+\frac{\gamma-1}{2} M^{2}\right]^{m / 5}}$

where it has been assumed that $\mu \sim T^{m}$. (Arguments about the appropriateness of variable properties corrections in general, and this reference condition in particular, for severely cooled boundary layers are presented in Section II D.) Finally, by assuming that the local mass flux is related to that at the throat by the local area ratio (i.e., one-dimensional flow), the convenient form of the equation for $h_{g}$ can be obtained

$$
h_{g}=\left[\frac{0.026}{D_{*}^{0.2}}\left(\frac{\mu_{0}^{0.2} c_{p}}{\operatorname{Pr}^{0.6}}\right)\left(\frac{p_{0} g}{c^{*}}\right)^{0.8}\left(\frac{D_{*}}{r_{c}}\right)^{0.1}\right]\left(\frac{A *}{A}\right)^{0.9} \sigma
$$

where the throat mass flux per unit area has been related to the rocket performance parameters characteristic velocity $c^{*}$ and chamber pressure $p_{0}$.

The success of this equation in fitting results from turbulent boundary-layer calculations over the whole nozzle when agreement is forced at the throat by selection of the constant $C$ is illustrated in Fig. 10. It is evident that the agreement can be made to be excellent except $n$ ear the entrance for a case in which the entrance boundary layer is thin (see Fig. 9). The weakness of the closed-form equation, of course, is in the uncertainty of the value of the constant $C$ for the flow of interest, since it is sensitive to the inlet boundary-layer conditions to the extent of about $10 \%$ at the throat. The value of $C$ as determined from the iterative simultaneous solution of the turbulent boundary-layer equations for "typically thick" inlet boundary-layer conditions (i.e., case 2a of Fig. 9) is 0.0225 , as compared with the value of 0.026 obtained from the first-approximation calculation and used in Reference 13. The worth of the closed-form equation (Eq. 50) lies in its simplicity, permitting the determination of the approximate $h_{g}$ distribution by a rapid slide-rule calculation. 


\section{Transport Properties}

It is evident from the analyses of Section $B$ that the problem under discussion involves all three of the transport properties usually found in heat-transfer problems, $\mu, \lambda$, and $\operatorname{Pr}_{r}$. If it is accep ted that the closed-form approximation is a reasonable over-all approximation to the results of the detailed boundary-layer analyses, then some idea of the impact of the transport properties on the problem can readily be obtained. In Eq. (48), it is seen that the transport properties have been combined in such a

way as to eliminate the direct use of $\lambda$, in the interests of convenience, leaving only the ratio of $\mu^{0.2 /}$ $P_{r}{ }^{0.6}$. Since in a theoretical calculation of high temperature viscosity and thermal conductivity, the same force constants and functional relationships are utilized for both $\mu$ and $\lambda$, it makes sense to cancel these factors out to the maximum extent possible in order to reduce the influence of uncertainties in the values. The final result, $\mu^{0.2} / \operatorname{Pr}_{r}^{0.6}$, is a parameter which is readily determined with fairly low uncertainty because of the low power exponent on viscosity compared with its range of uncertainty and the asymptotically constant characteristic of $\operatorname{Pr}$ at high temperatures.

A search of the literature for reliable, consistent values of the viscosity and Prandtl number (or thermal conductivity) reveals only a negligible amount of such data for a limited number of species above about $2000^{\circ} \mathrm{R}$ for viscosity and $1000^{\circ} \mathrm{R}$ for thermal conductivity and Prandtl number. The reason for this lack of information is that such measurements are very difficult to make. Surveys of the availability of data on high-temperature viscosity and Prandtl number as of 1958 and 1960 are reported in References 26 and 27 , respectively. Undoubtedly the most complete compilation of experimental transport properties for gases is contained in the NBS tables (28). As evidenced by the total absence of new experimental values of high-temperature transport properties, data of gases in the Thermodynamic and Transport Properties Symposia of 1959 (29) and 1.962 (30), the situation does not appear to have changed appreciably.

Thus, continuing to face this dearth of experimental data at high temperatures, one is forced to turn to theoretical calculations. For the most part, such theoretical calculations are essentially extrap* olation formulas derived from statistical mechanics models and based on two empirically determined constants. These constants, usually related to a collision diameter and to an attraction energy, are generally derived from room-temperature measurements of one of the transport properties. The results of an ambitious set of calculations such as these for 200 gases, covering the range of 100 to $5000^{\circ} \mathrm{K}$, are presented by Svehla (.31). Andrussow (32) reports simple correlations of the results of detailed 
statistical mechanical calculations of the transport properties of 34 gases (many of which were polar). He correlates the results by use of a series expansion of the exponent of the temperature dependence of the transport properties. In addition, he proposes some rather simple means for predicting the transport properties of mixtures of gases. Brokaw, (3.3) also presents a simple method for mixture calculations which utilizes alignment charts. Both of these techniques, as well as the earlier proposed technique of Buddenberg and Wilke (34), avoid the complexities required by the statistical mechanical methods of Hirschfelder, Curtiss, and Bird (35). Unfortunately, there are very few experimental data at high temperatures that would test the validity of any of these methods. A moderate temperature test of the method of Reference 34 indicated agreement within $10 \%$ for nitrogen-steam mixtures to $1200^{\circ} \mathrm{K}(36)$.

It was shown in Reference 26 that, consistent with the level of potential error introduced by approximations of Fq. (48), it is possible to make very simple, and hence rapid, approximations to the values of $\operatorname{Pr}$ and $\mu$. The approximation to the value of $\operatorname{Pr}$ results from the use of the same collision integrals for both viscosity and thermal conductivity. The equation for $\operatorname{Pr}$ on this basis is

$$
\operatorname{Pr}=\frac{\gamma}{1.94 y-0.74}
$$

where the ratio of mean-free paths for diffusion and viscosity was taken to be 1.2 for a smooth sphere model as opposed to 1.0, which is the Euken approximation used in Reference 13. The higher value of the ratio was found to agree better with available data (26). Svehla has presented a similar equation with slightly different constants:

$$
\operatorname{Pr}=\frac{y}{1.77 y-0.45}
$$

The latter agrees with the NBS air data (28) somewhat better in the range from 500 to $2000^{\circ} \mathrm{R}$, whereas from 2000 to $4000^{\circ} \mathrm{R}$ they both differ by about $2 \%$ from the Chapman - Cowling high-temperature prediction; i.e., $\operatorname{Pr}=0.715$.

Figure 2 of Reference 27 presented a plot of the one-fifth power of viscosity versus temperature of a large number of gases frequently present in rocket exhaust nozzles. Over the temperature range from 1000 to $8000^{\circ} \mathbf{R}$, the maximum deviation of $\mu^{1 / 5}$ of any of the se gases (except hydrogen) from that of air was only $8 \%$. The value for hydrogen was consistently about $15 \%$ lower than that for air. Thus, it was concluded that the value of $\mu^{1 / 5}$ of air could be taken as a reasonably accurate value for most 
combustion gas mixtures for use in calculations based on the closed-form approximation. For that purpose, a power-relation viscosity equation for air based on the NBS data was presented in Reference 1.3:

$$
\mu=\left(46.6 \times 10^{-10}\right)(\pi)^{1 / 2}\left(T^{\circ} \mathrm{R}\right)^{0.6} \mathrm{lb} / \text { inch sec }
$$

The calculated high-temperature values of Reference 31 for air are best fitted by

$$
\mu=\left(33.8 \times 10^{-10}\right)(\pi)^{1 / 2}\left(T^{\circ} \mathrm{R}\right)^{0.65} \mathrm{lb} / \text { inch sec }
$$

over the range from 1000 to $9000^{\circ} \mathrm{R}$. At most, the two equations differ by only $10 \%$ at the high-temperature end. Since there are no experimental data above about $2000^{\circ} \mathrm{R}$, the choice between these equations is arbitrary.

\section{Variable Properties}

By virtue of the fact that most rocket thrust-chamber walls are necessarily cooled severely with respect to the free-stream temperature and there is a region of supersonic flow (both conditions causing variations in the local static temperatures in the boundary layer), the question immediately arises as to how to adapt constant properties (i.e., $T_{w} \cong T$ ) correlations of skin friction and heat transfer to such problems. One method that has gained wide acceptance due principally to reports by Eckert (22) is the use of a reference temperature, at which the properties are evaluated in the constant properties correlations in order to adapt to calculations of heat transfer and skin friction for a boundary layer with large temperature variation. This procedure is widely accepted for adjusting for both the property variation due to cooling or heating and that due to compressibility. A mathematically equivalent procedure is to compute the constant properties $\bar{C}_{f}$ or $\bar{C}_{h}$ values by using the free-stream temperature properties and then to multiply the $\bar{C}_{f}$ or $\bar{C}_{h}$ by a function of the ratio of properties at the reference temperature to those at the free-stream static temperature.

$$
\begin{aligned}
& C_{f}=\bar{C}_{f}(\rho, \mu) \sigma^{\prime}\left(\frac{\rho_{\text {ref }}}{\rho}, \frac{\mu_{\text {ref }}}{\mu}\right) \\
& C_{h}=\bar{C}_{h}\left(\rho, \mu, c_{p}, P r\right) \sigma^{\prime}\left(\frac{\rho_{\text {ref }}}{\rho}, \frac{\mu_{\text {ref }}}{\mu}, \frac{{ }^{c}{ } \text { ref }}{c_{p}}, \frac{P r_{\text {ref }}}{P_{r}}\right)
\end{aligned}
$$


In most cases, both $c_{p}$ and $\operatorname{Pr}$ have been justifiably assumed not to vary significantly with the temperature variation across the boundary layer, giving the convenient result

$$
\frac{C_{f}}{\bar{C}_{f}}=\frac{C_{h}}{\bar{C}_{h}}=\sigma^{\prime}\left(\frac{\rho_{\text {ref }}}{\rho}, \frac{\mu_{\text {ref }}}{\mu}\right)
$$

For a laminar boundary layer, it can be shown that

$$
\sigma_{\text {laminar }}^{\prime}=\left(\frac{\rho_{\text {ref }} \mu_{\text {ref }}}{\rho}\right)^{1 / 2}
$$

whereas, for a turbulent boundary layer, the corresponding expression is

$$
\sigma_{\text {turbulent }}^{\prime}=\left(\frac{\rho_{\text {ref }}}{\rho}\right)^{3 / 4}\left(\frac{\mu_{\text {ref }}}{\mu}\right)^{1 / 4}
$$

or the alternative relationship suggested by Coles (Eq. 33). In Reference 9, $\sigma^{\prime}$ was modified slightly to permit viscosity evaluation at the stagnation temperature at the edge of the boundary layer and recognition of the dependence of $\theta$ on $(\rho U / \mu)^{-1 / 5}$, which alters the exponents of Eq. (57) to

$$
\sigma=\left(\frac{\rho_{\mathrm{ref}}}{\rho}\right)^{0.8}\left(\frac{\mu_{\mathrm{ref}}}{\mu}\right)^{0.2}\left(\frac{\mu}{\mu_{0}}\right)^{0.2}=\left(\frac{\rho_{\mathrm{ref}}}{\rho}\right)^{0.8}\left(\frac{\mu_{\mathrm{ref}}}{\mu_{0}}\right)^{0.2}
$$

Eckert has shown (22) that the most satisfactory definition of the reference state, accounting for both heating and cooling and compressibility effects at high speed, is

$$
T^{*}=0.5\left(T+T_{w}\right)+0.22 \operatorname{Pr}^{1 / 3}\left(T_{0}-T\right)
$$

which, for low-speed flow, reduces to

$$
T_{\text {low-speed }}^{*}=T_{a m}=0.5\left(T+T_{w}\right)
$$


This is also sometimes referred to as the film temperature. Although Eq. (60) was adopted initially in References 9 and 13 in order to be consistent with skin-friction and heat-transfer correlations then in use, its continued use is probably questionable in view of the analytical and experimental results that have since led to widespread use of Eq. (59). Interestingly, however, it will be shown in Section E that a variable properties correction based upon the arithmetic mean temperature (Eq. 60) seems to show the best agreement with one set of data at high Mach numbers for heated-air experiments. Although this result may not be general and the differences are not large, the use of Eq. (60) has been retained in calculations made here in order to maintain some degree of consistency with earlier published calculations. At a Mach number of $4,\left(T_{w} / T_{0}\right.$ of $\left.1 / 2\right)$, the highest $M$ for which data are available, the value of the variable properties correction factor based upon $T_{a m}$ is $20 \%$ above that based on $T^{*}$.

While the $T^{*}$ or $T_{a m}$ treatment of the variable properties problem has gained wide acceptance, it is worthwhile to re-examine the premises, data, and theoretical solutions upon which it is based. As far as this author can determine, the reference-temperature concept was initiated by Rubesin and Johnson (37). They found that all of the $C_{f}$ results of Crocco (38) (numerically solved from the laminar boundarylayer equations for a flat plate for $\operatorname{Pr}=0.725, M=0$ to $5, T_{w} / T=1 / 4$ to 2 , viscosity - temperature exponents $m=0.5,0.75,1.00,1.25)$ could be uniformly reduced to values within $1 \%$ of the constant properties value $\left(\bar{C}_{f} \sqrt{R_{x}}=0.664\right.$ ) by using a factor $\sigma^{\prime}$ (as in Eq. 56) and a reference temperature $T^{\prime}$ having constants only slightly different from those of Eq. (59). Significantly, the maximum value of $\sigma^{\prime}$ (corresponding to $M=0, T_{w} / T=1 / 4, m=0.75$ ) was only 1.07 , whereas the corresponding value of $\sigma^{\prime}$ for a turbulent boundary layer would be 1.357 , indicating the greater sensitivity of the turbulent boundary layer to such a correction. Young and Janssen (39) later solved the flat-plate laminar boundary-layer equations numerically out to higher Mach numbers and with cooler walls, using a Sutherland viscosity law rather than a simple power relation. By use of the reference temperature $T^{*}$ (Eq. 59), Eckert was able to correlate all of the Young and Janssen $C_{f}$ data to the constant properties value to within $2.6 \%$. When he tried the same thing on the $C_{h}$ data, deviations running from $-14 \%$ to $+9 \%$ were encountered. Later laminar boundary-layer calculation results by Van Driest (40), in which an enthalpy driving potential was utilized in the $C_{h}$ definition, were found by Eckert to be correlatable with the constant properties value within a few percent using Eqs. (56) and (59). When the Young and Janssen $C_{h}$ results were recorrelated on this basis, all were converged to the constant properties value within $2.6 \%$ except for one set of results calculated for $T$ of $100^{\circ} \mathrm{R}$. For these data, the correlation was made poorer by the $T^{*}$ correction, differing by from 14 to $20 \%$. The difficulty was attributed to peculiar transport properties variations at the low temperature. 
The way in which the $T_{\text {ref }}$ variable properties correction was carried over to the turbulent boundary-layer case is of interest. In the concluding remarks of Reference 39 , the statement is made: "More important, there are indications that this procedure ( $T$ ' properties evaluation) can be applied approximately to the turbulent boundary layer. That is, use the incompressible relations for the turbulent heat-transfer and skin-friction coefficients and the following relation for $T^{\prime}$ :

$$
\frac{T^{\prime}}{T}=1+0.032 M^{2}+0.58\left(\frac{T_{w}}{T}-1\right) \text { for } M<5.6 \text {, }
$$

The "indications" referred to are not discussed in the paper nor in any of the references of the paper. Eckert, $(41,22)$ recommended the reference-temperature variable properties correction for turbulent boundary layers based on the Young and Janssen statement, some limited skin-friction data obtained with a cooled wall, and reference to the NACA investigation of turbulent air flowing in tubes (42), in which some success in correlating the heat-transfer results with large temperature differences had been achieved using the film-temperature correction. It should be noted that in Reference 42 , although the film-temperature correction was apparently quite satisfactory in collapsing to the constant properties line, the majority of the data were obtained with heat addition. The performance of the film-temperature correction was quite inconclusively indifferent with respect to the limited data obtained with heat extraction. Furthermore, all of the data of Reference 42 were correlated on the basis of property values averaged over the length of the tube. This includes the obviously hazardous procedure of taking the numerical average of a density changing by as much as a factor of ten from inlet to outlet. Thus, these data (particularly those obtained with heat extraction) cannot be looked upon as a conclusive demonstration of the validity of the film-temperature correction for turbulent flow. Deissler (43) and Van Driest (44) almost simultaneously published solutions of the turbulent boundary layer for flow in tubes and over flat plates, respectively, in which uniform distributions of heat flux and shear stress across the boundary layer were assumed and ${ }^{-}$ a mixing length theory employed, together with an assumption of a turbulence Prandtl number of unity. Deissler, using a two-zone integration of the boundary-layer equations, obtained a result qualitatively different with respect to the variable properties question from that obtained by Van Driest, who used the same eddy transport relation all the way across the boundary layer. It was found by Deissler that his low-speed (incompressible) skin-friction and heat-tran sfer coefficient results with heat transfer could be reduced to the constant properties result within a percent or so by employing the film temperature $T_{a m}$ of Eq. (60) for property evaluation. This success encouraged the use of the film temperature in 
correlating the results of Reference 42. Van Driest's results could not be so correlated inasmuch as the influence of wall cooling according to his analysis was quite sensitive to the Reynolds number and $M$. At some Reynolds number for each $M$ (about $2 \times 10^{6}$ for $M=10$ ), the curves for different $T_{w} / T^{\prime}$ crossed, indicating a qualitatively different effect of wall cooling on either side of this Reynolds number. Going back even earlier than the analyses that led to the reference-temperature method, one encounters the Colburn equation for flow in smooth tubes, which is based on film-temperature evaluation of properties (45). While it is true that the Colburn film-temperature is equivalent to $T_{a m}$ of Eq. (60) when $T$ is replaced by $T_{b}$, it is significant that the Colburn equation is based on a density evaluated at the bulk temperature rather than the film temperature. Hence, the equivalent expression for $\sigma^{\prime}$ from the Colburn equation is

$$
\sigma_{\text {Colburn }}^{\prime}=\left(\frac{\mu_{f}}{\mu_{b}}\right)^{0.2}\left(\frac{P_{b}}{P_{f}}\right)^{2 / 3}
$$

which predicts that for gases with $P_{r}$ insensitive to temperature, the heat transfer is lower than the constant properties value in the case of wall cooling. The Colburn equation was used to correlate data for both gases and liquids. Its success was not critically tested by the available data, which generally had an experimental scatter of $\pm 40 \%$, masking the effects of properties variation.

Later, more definitive experimental results appeared which tend to disagree with the referencetemperature concept for predicting heat transfer and skin friction with cooled walls. Lobb, Winkler, and Persh, (18) reported results of the influence of heat transfer on skin friction at $M=5.0$ and 6.8 and showed that for $T_{w} / T_{a w}$ as low as 0.5 the skin-friction coefficient values were essentially equal to or slightly lower than those for an adiabatic wall. The reference-temperature correction (Eqs. 57, 59) would have predicted values 31 and $35 \%$ higher than the adiabatic wall skin-friction coefficients for $M=5.0$ and 6.8, respectively. Zellnik and Churchill (21) have reported local heat-transfer data from the inlet region of circular tubes with air entering at temperatures from about 500 to $2000^{\circ} \mathrm{F}$ and the wall maintained at about $100^{\circ} \mathrm{F}$ by water cooling. It was found that data obtained with $T_{w} / T_{b}$ down to about 0.25 agreed with the accepted constant properties values and showed no discernible influence of the ratio $T_{w} / T_{b}$, within the experimental scatter of $\pm 15 \%$, when properties were evaluated at the bulk temperature. Similar results were also reported by Wolf $(20)$ with both air and carbon dioxide transferring heat to cooled entrance regions of pipes over a range of $T_{w} / T_{b}$ from 0.3 to 0.7 . Wolf's local data, reproduced in Fig. 4 
of this Chapter, could al so be correlated with no effect of $T_{w} / T_{b}$, within the experimental scatter of $\pm 10 \%$, when properties were evaluated at the bulk temperature. While the data of References 21 and 20 were obtained principally with turbulent flow, Kays and Nicoll (46) have reported length - mean heattransfer data in circular tubes with low-speed laminar flow over a range of $T_{w} / T_{m}$ from 0.85 down to 0.55 , where $T_{m}$. is the $\log$ mean bulk temperature. These data were found to be equal to $95 \%$ of the constant properties value and were also independent of the ratio $T_{w} / T_{m}$, when the properties were evaluated at $T_{m}$. It must be noted, however, that the magnitude of the predicted variable properties correction is only slightly more than the $5 \%$ scatter.

While these data are probably insufficient to settle the question of the effect of property variation on heat transfer and skin friction definitively, they certainly cast suspicion on the validity of the reference-temperature methods as applied to severely cooled walls. In the author's opinion, there probably should be no correction made to the constant properties values of $\bar{C}_{h}$ and $\bar{C}_{f}$ based on freestream properties except for that due to compressibility effects. Note that the arguments presented here have been based upon experimental data obtained with negligible flow acceleration. Laminar flow solutions for cold walls have indicated that the effect of flow acceleration is to lower predicted skinfriction coefficients below the zero acceleration-constant properties values. On the other hand, Stantonnumber predictions may be altered either above or below the zero acceleration-constant properties values, depending upon the viscosity - temperature relation selected. Thus, for accelerated laminar flow, cold walls further disturb the equality between $C_{h}$ and $C_{f} / 2$ predicted by Reynolds analogy. Lacking the capability of making similar calculations for the turbulent boundary layer, there is no alternative but to resort to intuitive arguments such as presented in Section IIA and then to check the results against the best data available. For turbulent boundary layers, a method for relating the value of $C_{f_{a}}$ for an adiabatic wall at high speeds to the constant properties value $\bar{C}_{f}$ has been suggested by Coles (15) and is presented in Appendix A of this Chapter. Calculations have also been made (and are presented here), however, which are based on the arithmetic mean temperature as a reference temperature. This has been done to illustrate the possible magnitude of the variable properties correction and to relate the results with previously calculated and correlated results. The fact that the data in some regions and under some conditions best fit the predictions based upon the arithmetic mean temperature may or may not be significant. Other factors at work, such as transition, free-stream turbulence, acceleration, etc., as will be discussed, may account for this behavior. Additional experiments in which boundary-layer details are determined by probing will be needed to sort out these effects. 


\section{E. Driving Potential}

\section{Without Chemical Reaction}

To this point, attention has been focused primarily upon the heat-transfer coefficient that part of the Newton cooling equation which is dependent upon the fluid motion. To complete the calculation of the heat flux, this coefficient must, of course, be multiplied by a driving potential which represents the difference of the energy levels between free stream and wall. The object of this "separation of variables" is to arrive at a coefficient which is independent of both the difference and the absolute levels of the energy in the free stream and wall. In the case of low-speed flow with small temperature difference between free stream and wall, this ideal separation of variables is possible, the driving potential in this case being simply the temperature difference

$$
q_{w}=\bar{h}_{g}\left(T-T_{w}\right)
$$

As the speed of the flow increases to the point at which there is an appreciable difference between freestream stagnation and free-stream static temperatures, it becomes evident that in order to maintain the separation of variables, one must modify the low-speed correlations of the heat-transfer coefficient as in Eq. (55) and also replace $T$ with a recovery temperature $T_{a w}$, the temperature the wall would attain if insulated:

$$
q_{w}=\bar{h}_{g} \sigma^{\prime}\left(T_{a w}-T_{w}\right)
$$

It should be noted that

$$
T_{a w}=T+R \frac{U^{2}}{2 c_{p}}
$$

where $R$, the recovery factor, has been shown by both theory and experiment (including flow with acceleration) to be equal to $\operatorname{Pr}^{1 / 3}$ for turbulent boundary layers $(47,48)$.

As the temperature difference and velocity increase to the magnitudes of interest to a cooled supersonic nozzle flow, the question of the appropriate value of $c_{p}$ in both $T_{a w}$ (Eq. 65) and $h_{g}$ (Eqs. 17 
and 48) becomes important. Van Driest (44), Eckert (22), and others have shown that the enthalpy difference is the fundamental measure of the energy level difference, or driving potential, and can, in general, be substituted for the product of $c_{p}$ and temperature difference; thus,

$$
q_{w}=\bar{h}_{i} \sigma^{\prime}\left(I_{a w}-I_{w}\right)
$$

where $h_{i}$ is $h_{g}$ with $c_{p}$ eliminated (e.g., Eqs. 17 and 48):

$$
l_{a w}=I+R \frac{U^{2}}{2}=I_{0}\left[1+(R-1) \frac{\frac{U^{2}}{2}}{I_{0}}\right]
$$

\section{With Chemical Reaction}

In recent years, the question of the effect of chemical reaction on the heat transfer has been received considerable attention. In particular, for both high-speed stagnation point flow and flow of high-energy rocket-engine combustion gases, an appreciable mole fraction of dissociated species such as $\mathrm{N}, \mathrm{O}, \mathrm{H}, \mathrm{OH}, \mathrm{F}$, etc., is present in the free stream. Upon being brought to rest against a cooled wall, the natural tendency toward chemical equilibrium results in very energetic exothermic recombination reactions. If the local conditions are such that these reactions are very slow with respect to specie residence times near the wall, the flow is said to be chemically frozen, and no effect on heat transfer is to be expected, provided the wall is noncatalytic to the recombination reaction. If, at the other extreme, the conditions are such that the reactions are so very fast that the flow is able to maintain local chemical equilibrium every where within the boundary layer, then an enhancement in the heat transfer is to be expected as a result of the energy released by the recombination. Two other intermediate conditions are also of interest: (a) a boundary layer which is chemically frozen but is in contact with a catalytic wall of such activity that all dissociated species diffusing to the wall are immediately recombined and (b) a boundary layer with intermediate arbitrary recombination rates both within the flow and at the wall. Because of the extensive treatment of these effects on heat transfer by Denison and Dooley (49), Lees (50), Rosner (6), and others, it will suffice here to give only the results as they apply to the rocket nozzle case and to discuss the possible magnitudes of the effects.

Before proceeding to the general result that will apply for the cases described above, it is 
important to establish the influence of chemical reaction on heat transfer in the special case in which the parameter $\rho D_{12} \bar{c}_{p} / \lambda$ is unity. According to Lees (50), when a gas contains more than one chemical species, heat is transported not only by heat conduction but also by diffusion currents carrying both thermal and chemical enthalpy. In two-dimensional or axially symmetric flows of boundary-layer type, the rate of heat transport across stream lines in laminar flow is given by

$$
q=-\lambda \frac{\partial t}{\partial y}+\Sigma \rho k_{i} v_{i} i_{i}
$$

where $i_{i}$ is the local static enthalpy:

$$
i_{i}=\int_{0}^{t}{ }^{c_{p_{i}}} d t+I_{i}^{(0)} ; \text { and } i_{0}=\sum_{i} k_{i_{i}} i_{i}+\frac{u^{2}}{2}
$$

and where $I_{i}^{(0)}$ is the heat of formation of the $i$ th species. In the special case of a binary mixture, Fick's law states that

$$
\rho k_{i} v_{i}=-\rho D_{12} \frac{\partial k_{i}}{\partial y}
$$

Thus, Eq. (68) becomes

$$
q=-\left[\lambda \frac{\partial t}{\partial y}+\rho D_{12} \Sigma_{i} \frac{\partial k_{i}}{\partial y}\right]
$$

The complete static enthalpy, which includes both the thermal and chemical enthalpies of the mixture, is defined by $i=\Sigma k_{i} i_{i}$, so that $d i=\bar{c}_{p} d t+\sum i_{i} d k_{i}$, where $\bar{c}_{p}=\Sigma k_{i} c_{p_{i}}$. Thus, substituting in Eq. (71), the expression for $q$ becomes

$$
q=-\frac{\lambda}{\bar{c}_{p}}\left[\left(\frac{\partial i}{\partial y}-\Sigma i_{i} \frac{\partial k_{i}}{\partial y}\right)+\frac{\rho D_{12} \bar{c}_{p}}{\chi} \Sigma i_{i} \frac{\partial k_{i}}{\partial y}\right]
$$


where the first two terms are due to conduction and the third to diffusion. From Eq. (72), it is clear that when the parameter $\rho D_{12} \bar{c}_{p} / \lambda$ is unity, the heat transfer is given by

$$
q=-\frac{\lambda}{\bar{c}_{p}} \frac{\partial i}{\partial y}
$$

from which Lees draws the conclusion that the net heat flux is then independent of the mechanism of heat transfer and of the chemical reaction rates in the mixture. The parameter $\rho D_{12} \bar{c}_{p} / \lambda$ has been designated as the Lewis - Semenov number and given the symbol $L e_{f}$. Although this result has been derived for a binary mixture in a laminar boundary layer, both Lees (50) and Rosner (6) imply that the same result can in principle be derived for a multicomponent reacting gas mixture by using the generalized conservation equations as presented by Hirschfelder (35). However, to make the problem more tractable, Lees (50) considers a gas mixture consisting of two groups of species, each with about the same atomic or molecular weight and about the same mutual collision cross-sections. He asserts that these can be replaced by an effective binary mixture, in which each group acts like a single component so far as diffusion is concerned. The enthalpy of each individual species must be carefully distinguished in calculating the energy transport, but there is only one effective diffusion coefficient, and Fick's law is applicable. It would appear that this approximation is equally applicable to the chemical recombination within a rocket nozzle. As for the applicability of the result to the turbulent boundary layer, Lees (50) presents a series of plausible arguments and assumptions, as rigorously convincing as any on the turbulent boundary layer, which suggest that as long as some form of Reynolds analogy is still applicable, results for the reacting laminar boundary layer are applicable to the turbulent boundary layer. The molecular Lewis - Semenov number appears in the turbulent reacting boundary-layer equations just as the molecular Prandtl number appears in the turbulent nonreactive boundary-layer equations because of the calculation of the total thermal resistance as the sum of that in a laminar sublayer, a buffer layer, and a turbulent core for both the conductive and diffusive energy flux, similar to that made by von Kármán in the nonreactive case.

For the special case of a frozen laminar boundary layer and a catalytic wall, as described above, Lees (50) and Rosner (6) have obtained the result 


$$
q_{w}=h_{i}\left(I_{0}-I_{w}\right)\left\{1+\left[\operatorname{Pr}_{f}^{1 / 2}-1\right] \frac{\Delta I_{\text {kinetic }}}{\left(I_{0}-I_{w}\right)}+\left[L e_{f}^{2 / 3}-1\right] \frac{\Delta I_{\text {chem }}}{\left(I_{0}-I_{w}\right)}\right\}
$$

where $I_{0}$ is the free-stream value of $i_{0}$ as given by Eq. (69), $\Delta I_{\text {kin etic }}$ is $U^{2} / 2$ and $\Delta I_{\text {chem }}$ is $\sum_{i}\left(K_{i}-K_{i}\right) l_{i}^{(0)}$. When $L e_{f}$ is unity, Eq. (74) reduces to

$$
q=h_{i}\left(I_{a w}-I_{w}\right)
$$

as one should expect from Eq. (73). Note, however, that Eq. (75) is not identical to Eq. (66) except in the special case in which $\Delta I_{\text {chem }}$ is zero.

In order to generalize the driving potential for a reacting multicomponent laminar boundary layer for a Lewis-Semenov number other than unity, Rosner (6) has made use of the energy equation as written by Lees $(50)$ in the following form:

$$
\rho\left[u \frac{\partial i_{0}}{\partial x}+v \frac{\partial i_{0}}{\partial y}\right]=\frac{\partial}{\partial y}\left[\frac{\mu}{\operatorname{Pr}_{f}} \frac{\partial i_{0}}{\partial y}+\mu\left\{1-\frac{1}{\operatorname{Pr}_{f}}\right\} \frac{\partial 1 / 2 u^{2}}{\partial y}+\sum_{i} \rho D_{i m}\left\{1-\frac{1}{L e_{f, i}}\right\} i_{i} \frac{\partial k_{i}}{\partial y}\right]
$$

where $\operatorname{Pr}_{f}$ is the "frozen" Prandtl number of the multicomponent mixture, $D_{i m}$ is the diffusion coefficient of the $i$ th species with respect to the mixture, $L e_{f, i}$ is $\left(\rho D_{i m} \bar{c}_{p}, f\right) / \lambda_{f}$, and $i_{0}$ is given by Eq. (69) and contains chemical enthalpy. By making the approximation (see derivation of Eq. 33, Reference 50)

$$
\Sigma i_{i} \frac{\partial k_{i}}{\partial y} \approx \Sigma l_{i}^{(0)} \frac{\partial k_{i}}{\partial y}
$$

which neglects the differences in thermal enthalpy between components compared with the heats of formation (usually highly accurate for mixtures of interest), Rosner (6) has rewritten Eq. (76) as follows: 


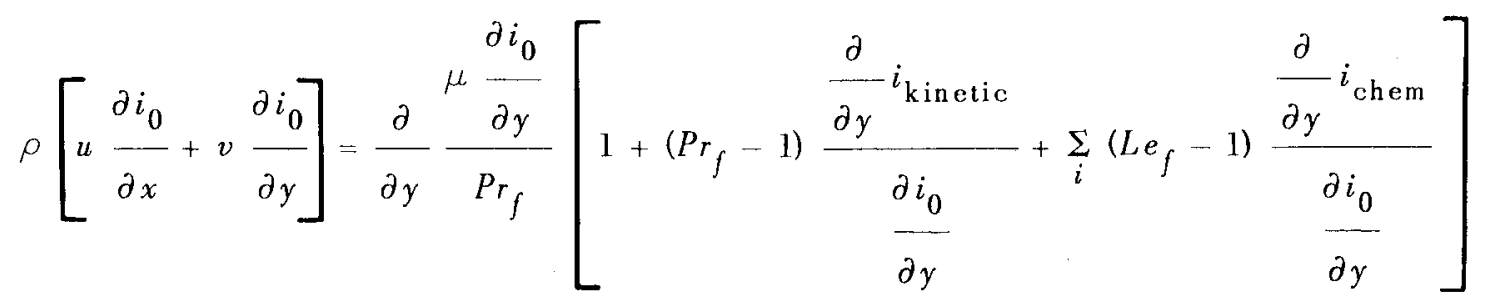

where $i_{\text {kinetic }}=u^{2} / 2$ and $i_{\text {chem }}=I_{i}^{(0)} k_{i}$. Noting the formal similarity between the general energy equation (as written in the form of Eq. 78) and Eq. (74) for its special case, Rosner (6) has proposed a generalized driving potential of the form

$$
q=h_{i}\left(I_{f, R}-I_{f, w}\right)
$$

where the subscript $R$ is for recovery, $f$ for frozen, and the value of $I_{f}=I_{0}-\Delta I_{\text {kinetic }}-\Delta I_{\text {chem }}=$ $\sum_{i} K_{i} \int_{0}^{T} c_{p_{i}} d T$ is computed for the different equilibrium compositions existing, respectively, at the edge of the boundary layer and at the wall. The relation between Eqs. (77), (76), and (74) becomes evident by expanding $I_{f, R}$; i.e.,

$$
q=h_{i}\left[I_{0}+(R-1) \Delta I_{\text {kinetic }}+\sum_{i}\left(R_{c, i}-1\right) \Delta I_{\text {chem, } i}-I_{f, w}\right]
$$

or, equival ently,

$$
q=h_{i}\left[I_{f}+R I_{\text {kinetic }}+\sum_{i} R_{c, i} \Delta I_{\text {chem }, i}-I_{f, w}\right]
$$

where it is noted that $u=0$ at the wall so that $\Delta I_{\text {kinetic }}=I_{\text {kinetic }}$, and where $R_{c, i}$ is a chemical recovery factor dependent upon $L e_{f, i}$. It is suggested that the dependence of $R_{c}$ upon $L e_{f}$ will take a slightly different form from one flow to another just as, for example, $R$ for a laminar boundary layer is equal to $P_{r}^{1 / 2}$ and for a turbulent boundary layer it is equal to $P_{r}{ }^{1 / 3}$. It was already shown in Eq. (74) that for a frozen laminar boundary layer, $R_{c}$ is equal to $L e^{2 / 3}$. Fay and Riddell (5l) who carried out numerical calculations of heat transfer to a stagnation point in air with dissociation, found that their results were correlated well with $R_{c}$ given by $L e^{0.52}$ for equilibrium and by $L e^{0.63}$ for a frozen boundary layer with a catalytic wall. For turbulent boundary layers, Lees $(50)$ argues that $R_{c}$ by Reynolds analogy is dependent upon Le to the negative of the same power that $h_{g}$ is dependent upon Pr. Hence, from Eq. (48), it is concluded that $R_{c}$ is equal to $L e^{0.6}$, whereas from Eq. (A-8) it would appear that $R_{c}$ is also $R_{\theta}$-dependent. 
As for the effects on the heat transfer to be anticipated when recombination rates are finite at the wall or in the boundary layer (or both), Rosner (6) has presented qualitative arguments which suggest that in no case will the finite rates result in heat transfer either below the frozen case with the noncatalytic wall or above the complete equilibrium case. He notes that the heat-transfer rate becomes insensitive to the gas-phase reaction rates when the surface recombination rates become very large for a catalytic wall. Analogously, as one would expect, the heat-transfer rate becomes insensitive to surface recombination rates when the gas-phase recombination rate becomes large since no atomic species reach the wall. As mentioned in the previous paragraph, the heat transfer in these two limits is nearly equal. For a low recombination rate, the heat transfer approaches the chemically frozen case as the catalytic activity of the wall approaches zero. For a noncatalytic wall, the heat transfer approaches that of full chemical equilibrium as the chamber pressure, and, hence, homogeneous reaction rates increase. Unfortunately, since few reaction rate data are available against which to compare results, it is not possible at present to make calculations with confidence of the effect on heat transfer of finite rates. Thus, it is recommended that the equilibrium limit (Eq. 80 or 81 ), with $R_{c}$ equal to $L e_{f}^{0.6}$, be utilized in computing rocket thrust-chamber heat flux. This is probably well justified by the high pressures prevalent in the high heat-flux regions of most rocket thrust chambers. Of course, it remains to be demonstrated with definitive local data whether or not the accounting for chemical reaction suggested here actually applies for accelerating turbulent boundary layers with variable properties and streamwise varying species concentrations.

The maximum influence on heat transfer of the chemical recovery will probably be encountered with one of the most energetic chemical rocket propellant combinations such as $\mathrm{H}_{2}+\mathrm{O}_{2}$ at its maximum performance mixture ratio of 5 ; or in the case of the nuclear rocket, with hydrogen as the working fluid, both with nozzle walls at maximum temperature (say, about $1500 \mathrm{~K}$ for $\mathrm{H}_{2}$ regenerative cooling). Under these conditions, at $10 \mathrm{~atm}, \Delta I_{\mathrm{chem}} /\left(I_{0}-I_{f, w}\right)$ for $\mathrm{H}_{2}+\mathrm{O}_{2}$ would be about $15 \%$, whereas for the nuclear rocket it would be only about $7 \%$. The value of $L e_{f}$ for $\mathrm{H}$ atom diffusion through equilibriumdissociated hydrogen is given by Rosner (6) as 1.3 at these conditions. Thus, the heat-transfer enhancement due to chemical reaction for the nuclear rocket case from $\mathrm{Eq}$. (81), with $R_{c}=L e_{f}^{0.6}$, amounts to only about $9 \%$, of which only $1 \frac{1}{2} \%$ is due to $L e_{f}>1$. The value of $L e_{f}$ for $\mathrm{H}$ atom diffusion through water vapor, the major combustion gas constituent for the $\mathrm{H}_{2}+\mathrm{O}_{2}$ case, has been suggested by Rosner (6) as being conservatively as high as 4.0. The principal reason for the high $L e_{f}$ is the large imbalance in molecular weight between the $\mathrm{H}$ atoms and the water vapor. Even with this large value of Lee for $\mathrm{H}$ atoms, 
the net enhancement of the heat transfer over that of a frozen boundary layer with a noncatalytic wall is not as great as one might suspect because of the $15 \% \Delta l_{\text {chem }} /\left(I_{0}-I_{f, w}\right)$, only $8 \%$ is due to $\mathrm{H}$ atom recombinations, the other $7 \%$ being the result of reactions forming $\mathrm{H}_{2} \mathrm{O}$. The energy release from the latter processes while comparable to that of the $\mathrm{H}$ atom, will be limited by the diffusion of the much heavier species $\mathrm{O}$ and $\mathrm{OH}$, which, diffusing through $\mathrm{H}_{2} \mathrm{O}$ vapor, will probably have values of $L e_{f}$ near unity, as will the $\mathrm{H}_{2} \mathrm{O}$ vapor itself according to Rosner (private communication). Thus, virtually all of the $12 \%$ enhancement in heat flux due to $L e_{f, i} \neq 1$ results from the $\mathrm{H}$ atom reaction. The total enhancement in heat flux over that for a completely frozen boundary layer with noncatalytic wall is $32 \%$. It must also be remembered that the $15 \% \Delta I_{\mathrm{chem}} /\left(I_{0}-I_{f, w}\right)$ resulted from $100 \%$ of theoretical performance, with a wall at $1500^{\circ} \mathrm{K}$, at $10 \mathrm{~atm}$ pressure. This fraction will fall rapidly with reduced combustion efficiency (it is down to $7 \%$ at $95 \%$ combustion efficiency) and with reduced wall temperatures and increased pressures.

It is interesting to note that when $L e_{f}$ is unity, or when the enhancement due to $R_{c} \neq 1$ is to be neglected in a calculation, it is unnecessary to determine $\Delta I_{\mathrm{chem}}$. For such a situation, the value of $I_{0}$ can be calculated by a method described by Welsh and Witte (52), in which it is unnecessary to solve the simultaneous species equilibrium equations. Hence, a calculation can be made with a slide-rule in a few minutes. The method is based upon the assumption that the species at equilibrium at the wall condition are readily known from the initial reactants and that the wall is sufficiently cool to preclude the existence of any dissociated species in equilibrium there. (This situation pertains for cooled walls for relatively simple reactants such as $\mathrm{H}_{2}+\mathrm{F}_{2}$ and $\mathrm{H}_{2}+\mathrm{O}_{2}$. For a system such as $\mathrm{N}_{2} \mathrm{O}_{4}+\mathrm{N}_{2} \mathrm{H}_{4}$, for example, some assumption about the presence or absence of $\mathrm{NH}_{3}$ must be made but introduces only small differences.) The method is possible because the total energy available to the system is exactly equal to the heat of reaction, which is known from the heat of formation of the reactants and the products. The equation for $I_{0}-I_{f, w}$ from Reference 52 , converted from volume to mass units and translated to the nomenclature of this Chapter, is

$\left(I_{0}-I_{w, f}\right)=\left[\sum_{i} K_{j} I_{j}^{(0)}-\sum_{i} K_{i_{\text {ref, } w}} I_{i}^{(0)}\right]+\sum_{j} K_{i} \int_{T_{\text {ref }}}^{T_{\text {inj }}}{ }^{c}{ }_{p_{j}} d T-\Sigma K_{i_{\text {ref }, w}} \int_{T_{\text {ref }}}^{T}{ }^{T} c_{p_{i}} d t$

where subscript $j$ refers to reactant species and $i$ to product species. Since heats of formation $I_{i, j}^{(0)}$ are commonly tabulated at $298^{\circ} \mathrm{K}$, this is a convenient temperature to take as $T_{\text {ref }}$. If the species $K_{i}$ are 
known because of the assumption of no dissociated species at the wall, then they are also the same at $T_{\text {ref }}$ since generally $T_{\text {ref }}<T_{w}$. The third term of Eq. (82) represents a difference in enthalpy between the reactant constituents at $T_{\text {inj }}$ and $T_{\text {ref }}$. If $T_{\text {inj }}$ is sufficiently close to $T_{\text {ref }}$ (as it usually is), no appreciable dissociation or phase change occurs between these temperatures, permitting the use of a simple expression for the sensible enthalpy change such as that used in Eq. (82). The fourth term represents the change of enthalpy of the product constituents between $T_{\text {ref }}$ and $T_{w}$. Again, if no dissociation or phase change occurs between these temperatures, a simple expression for the sensible enthalpy change such as appears in Eq. (82) is permissible. If dissociation or phase change does occur, the final term must be modified accordingly, degrading the simplicity of the equation. While Eq. (82) was derived for $100 \%$ of theoretical combustion performance, it is shown in Reference 52 that by multiplying the two bracketed terms by the ratio of the square of the actual $c^{*}$ to the $100 \%$ (or ideal theoretical) $c^{*}$, Eq. (82) can be adapted for $c^{*}$ lower than $100 \%$.

\section{AIR EXPERIMENTS}

\section{A. Purpose}

At a very early stage in the investigation of the problem of heat transfer in rocket nozzles, it was realized that it would be essential to obtain some heat-tran sfer data in nozzles operating under controlled conditions for which the flow would be describable. This need suggested experiments with heated air flowing through cooled supersonic nozzles for the purpose of eliminating combustion effects such as large secondary flows, nonlinear oscillations, variable total temperature, recombination, excessive free-stream turbulence, and uncertain transport properties. With hot-air experiments, it is still possible to retain the essentials of the problem analytically modeled in Section II; i.e., rapidly accelerating turbulent boundary layers, variable properties, and variable flow origin. 


\section{B. Literature}

Even with the general recognition of the significance of such experiments for many years, relatively few significant results of such experiments are cited in the literature. The reason for this paucity of data is that a rather large air supply and a method of heating the air are required and that measuring local heat flux from a hot gas to a cold wall in a region in which the heat flux varies rapidly in the flow direction is experimentally difficult. The earliest experiments of this type reported were those of Saunders and Calder (53), followed later by Ragsdale and Smith's (54). Unfortunately, the nozzles used in both of these investigations were built with convergent and divergent half-angles of about $1 \mathrm{deg}$, so that there was negligible acceleration to the flows. Baron and Durgin (55) obtained experimental data in a two-dimensional nozzle at a stagnation pressure range of 6 to $30 \mathrm{psi}$ at $570^{\circ} \mathrm{R}$. They succeeded in correlating very low heat-flux data from low pressure-gradient air flow with turbulent boundary-layer flat-plate correlation equations to $\pm 25 \%$ after considerable manipulation of the "effective flow origin" and of effects of surface-temperature variation. Their raw or "uncorrected" data tended to fall 50 to $70 \%$ below a Colburn analogy correlation for a flat plate, i.e., $S t=0.0374\left(R_{x}\right)^{-1 / 5}$ for supersonic regions of the nozzle, but showed fair agreement in the subsonic region. It wasn't until still later that investigations were made for the specific purpose described in Section III A by Massier (56), Kolozsi (57), Fortini and Ehlers (58), and Back, Gier, and Massier (59). It is the latter of these references that will form the principal basis for discussion in the remainder of this Section, although brief comments will be made about the results of the Kolozsi and of Fortini and Ehlers. Kolozsi obtained data with a $7 \frac{1}{2}$-deg half-angle convergent - divergent nozzle at $1200^{\circ} \mathrm{R}$ at stagnation pressures of 226 and 370 psia. Fortini and Ehlers obtained data with a nozzle having a 30 -deg half-angle convergence and a Rao-design divergent section at $1600^{\circ} \mathrm{R}$ and 300 psia stagnation pressure. The data of Back, Gier, and Massier were obtained with a 30-deg half-angle convergent, 15-deg half-angle divergent nozzle over a range of stagnation temperatures from 1000 to $2000^{\circ} \mathrm{R}$, and stagnation pressures of 30 to 250 psia. The wide range of operating conditions of Reference (59) makes the results of particular interest. 


\section{Experimental Techniques}

The flow system and instrumentation locations utilized in the investigation reported in Reference (59) are depicted in Fig. 11. The source of the heated air was a wind-tunnel compressor system, followed by a turbojet combustor can in which very lean mixtures of methanol and air were burned. The mole fraction of methanol required to reach even $2000^{\circ} \mathrm{R}$ was small enough that the resultant gas has thermodynamic and transport properties only slightly different from air. The fluid-dynamic effects of the combustion were adequately damped out by the low-velocity calming section and the system of baffles and screens followed by an aerodynamically contoured exit from the calming section. To prevent the unwanted growth of thick thermal boundary layers, the calming section and contoured exit were lined with inconel, and the system was designed so that the liner very nearly reached the stagnation temperature. By varying the length of the instrumented cooled duct downstream of the settling chamber, the boundarylayer conditions at the nozzle inlet could be varied. Free-stream temperature was measured by two shielded thermocouples just upstream of the nozzle inlet. Traverses were made of boundary layers about $1 \frac{1 / 4}{4}$ inch upstream of the nozzle entrance to determine the temperature and velocity profiles and boundarylayer thicknesses. Probe tip details are shown in Fig. 12. The nozzle utilized for all of the measurements of heat flux had an internal contour closely similar to that depicted in Fig. 5, only with a slightly larger throat radius of $0.902 \mathrm{inch}$. The static-pressure distribution along the wall of the nozzle was measured with thirty-two static-pressure holes of 0.040 -inch diameter, spaced both circumferentially and axially in the nozzle wall.

Steady-state wall temperatures and heat fluxes were determined from the output of thermocouples imbedded in cylindrical plugs, a typical one of which is shown in Fig. 13. Three thermocouples were formed along the length of each plug, which, when instrumented, was pressed into a hole drilled through the nozzle wall. By making the plugs from the same billet from which the nozzle was machined and using a force fit, the thermal disturbance to the wall was minimized. The material selected for the nozzle and plugs was type 502 stainless steel because of the known insensitivity'to temperature of its thermal conductivity. Measurements of the thermal conductivity for a sample from the billet used for the nozzle were obtained from the National Bureau of Standards. The locations of the thermocouples were determined 
to an accuracy of less than $1 \%$ of the inter-thermocouple spacing by a Kelvin bridge electrical technique described in detail in Reference 59. Twenty-two plugs were used to obtain the heat-flux distribution, with spacing made both axially and circumferentially. It can be shown that if the isotherms of the nozzle wall are essentially parallel (as they were experimentally shown to be in Fig. 14), the local heat flux can be determined from the local temperature gradient measured normal to the nozzle inner surface without the necessity of having to make any correction for axial conduction.

In the investigation reported in Reference 58 , the problem of axial conduction was fairly well circumvented by the use of thermally insulated, one-dimensional heat-conduction plugs. Both of these thermocouple plug techniques have been made to yield what are believed to be reliable and accurate data; however, not without considerable effort. As a consequence of these evaluations of the effort required, Back, et al. (59) are using calorimetrically cooled nozzles in continuing their air experiments. These are made of axially short sections, which permit measurement of the less desirable circumferentially averaged and axially semilocal values of heat flux. Nozzles of this type have been used extensively in obtaining measurements under rocket thrust-chamber conditions. An example of such a nozzle will be shown in connection with Section IV.

From this discussion, it is evident that it is much more difficult to measure local heat-transfer coefficients from a hot gas to a cool wall than to measure the wall temperature for a controlled local input of heat from a hot wall to a cool gas. The extra effort is apparently well justified for the purposes of the experiments under consideration here in view of the suspected different influence of variable properties between heating and cooling.

\section{Experimental Results}

\section{Static Pressure Distributions}

Measured wall static pressures from tests made at stagnation pressures ranging from 45 to 150 psia are presented in Fig. 15. Deviations from the one-dimensional-flow predicted wall static pressures are clearly evident and are obviously beyond the spread of the data. The deviations undoubtedly result from significant radial velocity components caused by the taper and curvature of the nozzle. In Fig. 16, the distribution of local mass flux per unit area at the edge of the boundary layer $\rho U$ computed from the wall static-pressure data and normalized by the mass flux per unit area predicted from onedimensional flow $\rho_{1} U_{1}$ is presented for 75-psia stagnation pressure. Since from the closed-form 
approximation equation (Eq. 48), $h_{g}$ is proportional to $(\rho U)^{0.8}$, deviations of the real flow $\rho U$ from the one-dimensional values of up to $15 \%$ result in errors of over $10 \%$ in $h_{g}$ if the one-dimensional $\rho_{1} U_{1}$ values are used. Deviations considerably greater than this in severely turned flows in the divergent portions of contoured nozzles have been predicted by method-of-characteristics flow analyses and have been observed by Fortini and Ehlers (58). In the transonic region of a nozzle, the prediction of the real flow is considerably less certain than for the fully supersonic region. Nevertheless, Oswatitsch and Rothstein (60) did consider two-dimensional flow in a convergent-divergent nozzle. The wall boundary layer was neglected, and it was required that the fluid velocity at the wall be exactly parallel to it. Their result of the ratio of the mass flux per unit area at the nozzle wall to that for one-dimensional flow is given by

$$
\frac{\rho U}{\rho_{1} U_{1}}=\frac{\left\{1-\frac{\gamma-1}{2}\left(M_{1} \frac{a_{1}}{a_{0}}\right)^{2}\left(\frac{U}{U_{1}}\right)^{2}\right\}^{\frac{1}{\gamma-1}}}{\frac{\rho_{1}}{\rho_{0}}} \frac{U}{U_{1}}
$$

where

$$
\frac{U}{U_{1}}=\sqrt{\left\{1+\frac{1}{2}\left[\frac{1}{2} r \frac{d^{2} r}{d z^{2}}+\frac{1}{4} \frac{\frac{d U_{1}}{d z}}{U_{1}} r \frac{d r}{d z}-\left(\frac{d r}{d z}\right)^{2}\right]\right\}^{2}+\left(\frac{d r}{d z}\right)^{2}}
$$

The predicted ratio $\rho U / \rho_{1} U_{1}$ from Eq. (83) is in fair agreement with the data presented in Fig. 16. The position of the sonic line is predicted to be somewhat upstream of the geometrical throat. Note the predicted discontinuity of the mass flux at the intersection of the conical sections of the nozzle with the circular arc throat region. F,rom results such as these, one concludes that if requirements of precision of the calculation of nozzle heat flux are sufficient to justify a boundary-layer (rather than a closed-form) calculation, then the deviations from one-dimensional flow should be determined and fed into the calculation. 


\section{Boundary Layers at Nozzle Inlet}

In order to determine the nature and thicknesses of the velocity and temperature boundary layers entering the nozzle, the boundary layers were probed just upstream of the nozzle inlet. Typical results of such measurements made with an approach length of about $3 \frac{1}{2}$ diameters are presented in Fig. 17 for $1500^{\circ} \mathrm{R}$ temperature and a range of stagnation pressures. A 1/7-power constant properties profile (as assumed in Section II) for both temperature and velocity is found to agree satisfactorily with the data. The thicknesses $\delta^{*}, \theta$, and $\phi$ were calculated using the thick boundary-layer relationships of Appendix $\mathrm{C}$ and are about $5 \%$ less than those predicted assuming the thin boundary-layer relations of Eqs. 9,12 , and 15 .

\section{Heat Transfer Results}

In Fig. 18, a composite presentation is made of raw heat-transfer coefficient data covering the full range of stagnation temperatures and pressures investigated. The majority of the tests were duplicated, with the results found to be reproducible to within $\pm 2 \%$. Some evidence of circumferential variations of greater extent than this can be seen in the Figure by observing symbols that are similarly tagged. Although circumferential nonuniformities were not evident from probing the free stream ahead of the nozzle, it is possible that they could have gone undetected in the boundary layer. The data from the several tests presented consistently show a maximum heat flux upstream of the geometrical throat very close to the point of maximum mass flux per unit area, as indicated in Fig. 16. The apparently strange behavior of the data near the nozzle exit for the lower stagnation pressures is due to separation.

In order to compare these results with distributions predicted from the turbulent boundary-layer calculation of heat flux presented in Section II A, the distributions from two tests have been selected as being typical of most of the remainder of the data. The data of which these results are not typical will be discussed separately. The two tests selected were both made at a stagnation temperature of about $1515^{\circ} \mathrm{R}$, one at a stagnation pressure of $75 \mathrm{psia}$ and the other at the highest stagnation pressure investigated -254 psia. The heat-transfer coefficient distributions from the two tests are presented in Figs. 19 and 20 , respectively. The four solid lines on the Figures are the "iterative simultaneous" turbulent boundarylayer solution results (Eq. A-8) obtained using the experimentally measured boundary-layer thicknesses at the nozzle inlet for the initial conditions and the experimentally determined $\rho U$ distribution (see Fig. 16). In four separate calculations, use was made of different combinations of the properties evaluation temperature and the interaction exponent $n$ (Eq. A-8). The properties alternatives selected were (a) 
density and viscosity evaluated at a reference temperature equal to arithmetic mean between wall and free-stream static (film) temperature (Eq. 60) and (b) properties evaluated in accordance with Coles' method of computing $C_{f}$ for an adiabatic wall (Eqs. A-1, A-2, A-3). The two alternatives for the boundarylayer interaction exponent $n$ were (a) 0.1 , which is about the value used in Reference 9 , where some physical justification for its selection was presented and (b) 0, i.e., the momentum and energy equations essentially decoupled. The lowest of the four curves, Curve D, is seen to be approximately $30 \%$ below curve $A$ in the throat region of both Figures. Curve $A$, based on $n$ of 0.1 and properties at $T_{a m}$, it should be pointed out, is based on the same assumptions as those behind the results presented in Reference 9 (and in Figs. 9 and 10), to which the closed-form equation (Eq. 50) was fitted. It is seen, however, that values from Eq. (50) lie above curve A, since curve A resulted from an iterative simultaneous solution, as discussed in Section II. The different relationship between curve A values and Eq. (50) values of $h_{g}$ in Figs. 19 and 20 is due to differing entrance boundary-layer thicknesses for the two sets of calculations. The dissimilarity of the shapes of the curves as shown in Fig. 10 is due to the use of experimental $\rho U$ values in the boundary-layer calculations and the use of $\rho_{1} U_{1}$ in Eq. (50). (More closely similar results could be obtained by using experimental $\rho U$ values in Eq. 48.) Significant (but not conclusive) is the agreement in the throat region between the data and curve $\mathrm{D}$, exhibited in both Figs. 19 and 20. In the contraction and expansion sections of the nozzle, the data were perhaps in equally good agreement with curve C. It is evident that unless a large quantity of such data is compared with predictions covering a wide range of operating conditions, differences of this order probably cannot be resolved in this manner.

The effect of varying nozzle-inlet boundary-layer thicknesses on the heat transfer is shown in Fig. 21 for a stagnation temperature of $1500^{\circ} \mathrm{R}$ over a range of stagnation pressures from 75 to 200 psia. With no cooled approach length (i.e., nozzle connected directly to contoured discharge nozzle of calming section), the inlet velocity boundary layer was determined by probing to be about $5 \%$ of the nozzle-inlet radius. The heat-transfer coefficients for tests made under this condition were consistently higher over most of the nozzle than those of tests in which an 18-inch cooled approach section was used. For these tests, it was determined by probing that the inlet boundary-layer thickness was up to about $25 \%$ of the nozzle-inlet radius. The trends of higher heat-transfer coefficients in the contraction and throat regions are consistent with predictions of the type shown in Fig. 9. The predicted smaller, but still persistent, differences in the divergent region are also observed. It is clear that in real flows of interest, it is unlikely that "near-zero" inlet boundary-layer thicknesses will be encountered, nor were they experimentally 
observed even with the "zero" length inlet duct. Rather, thicker boundary layers, such as those observed in the tests of Fig. 18, will be encountered.

As mentioned earlier in this Section, some of the data did not behave in a qualitatively similar manner to those of Figs. 19 and 20. Data from tests of this type are presented in Fig. 22 and are compared with a type-D prediction curve; i.e., $n=0$, properties evaluated in accordance with Coles' prediction of $C_{f}$ for an adiabatic wall. Note that although near the nozzle inlet, the data agree fairly well with the prediction curve, a short way into the nozzle, they begin to fall considerably below the prediction. Beyond the throat but before the separation point, the data rise closer to the prediction curve. Data exhibiting this behavior relative to predictions (type-D curve) were obtained only at the lower stagnation pressures where it was reproducibly observed. In order to get a better picture of this behavior and of the variation of the heat-transfer coefficient at intermediate pressures, the heat-transfer coefficient at a single axial station in the nozzle was correlated versus stagnation pressure for virtually all the tests made. These plots, it was found, could be made more general by (a) nondimensionalizing the heattransfer coefficient by dividing by $\rho U c_{p}$ and multiplying by $\operatorname{Pr}^{0.6}$; thereby forming the modulus $C_{h} P_{r}^{0.6}$ and (i) nondimensionalizing the stagnation pressure by converting it to its proportional equivalent $\rho U$, multiplying by the local diameter ${ }^{*} \mathrm{D}$ of the axial station of interest, and dividing by the viscosity at the edge of the boundary layer $\mu$, forming the modulus $R_{D}$. When this was done, increases in $R_{D}$ at a given station at constant stagnation temperature were in direct proportion to increases in stagnation pressure. The data from virtually every test made with an 18 -inch cooled approach duct are presented in this fashion in Fig. 23 for selected axial stations identified by their respective area ratios $A / A_{*}$ and axial position $z / L$. When these nondimensional coordinates are used, it is simple to draw a curve corresponding to the closed-form approximation equation (Eq. 48). This was done in Fig. 23, where $\sigma^{\prime}$ was computed for film-temperature properties evaluation for an appropriate range of $T{ }_{w} / T_{0}$ and also properties evaluated at $T_{a w}$. The dashed lines represent results from turbulent boundary-layer calculations $(n=0$, properties appropriate for adiabatic wall $C_{f}$ ). In general, at the higher $R_{D}$, the data tend to vary with $R_{D}$ in about the same qualitative manner (i.e., slope) as these predictions. In making quantitative comparisons between the data and the predictions in this higher $R_{D}$ (or parallel) region, cognizance should be taken of the typical spread of data presented, as in Fig. 18, believed due to some circumferential nonuniformities. The most uniform agreement with the data in the higher $R_{D}$ region was found to be the prediction

\footnotetext{
*Although a local boundary-layer thickness, $\delta, \theta$, or $\phi$ would have been preferable, it was not measured
} directly. Hence, the more convenient linear dimension D was used and justified by the arguments of Section II B. 
based upon Eq. (48), with $T_{\text {ref }}=T_{a w}$. Except at $A / A_{*}=1$, where such a prediction was about $30 \%$ high, agreement to within about $15 \%$ was observed at all $A / A *$ for which data were obtained. The most surprising trends exhibited in Fig. 23 occur at the lower $R_{D}$ (i.e., lower stagnation pressures), where significant but correlatable departures from the predictions were observed. This is the $R_{D}$ region typified by data such as those presented in Fig. 22. It should also be noted that at the highest subsonic area ratio, i.e., near the nozzle inlet, the slope of the data generally follows the predictions for the whole range of $R_{D}$. The low $R_{D}$ departure of the data persists through the throat and into the supersonic region. It could actually continue out to the nozzle exit. However, it was not possible to operate the nozzle at low stagnation pressures without separation at the highest supersonic-area-ratio stations.

One possible explanation for this behavior of the heat-transfer results is that because of either the extreme acceleration or the combination of acceleration and cooling, the entering turbulent boundary layer experienced a reverse transition back toward (but probably not all the way to) a laminar condition. At some point downstream in the nozzle, there is perhaps again a forward transition back to fully turbulent conditions. For the present, this must stand as merely a hypothesis, which, when checked against the currently available data, was not found to be violated. The hypothesis must now be checked by making detailed boundary-layer surveys in the maximum acceleration region. Because boundary layers are thinning out so rapidly in this region (at least according to the turbulent boundary-layer calculations), such measurements are difficult to obtain experimentally. Other investigators have observed unexpected trends accompanying the acceleration of turbulent boundary layers. The proposed hypothesis may well explain some of the anomalous variation of heat transfer with stagnation pressure in rocket thrust chambers reported in Reference 52. These data will be discussed in Section IV. In Reference 61, a turbulent boundary layer at the entrance of a supersonic nozzle was found to undergo transition to a nearly laminar one at the nozzle exit when the stagnation pressure was $4.3 \mathrm{psia}$. When the pressure was increased to 14.2 psia, a turbulent boundary layer was found at the nozzle exit. No boundary-layer or local heat-transfer measurements were made within the nozzle. In Reference 62 , it was reported that heat-transfer trends of the type under discussion here were also observed at lower pressure-gradient subsonic flow conditions. In that investigation (62), there was departure from fully turbulent flow throughout the acceleration region, as indicated by the linearity of the measured velocity profiles near the wall. In Section III E, a derivation is made of a parameter relating to the level of eddy transport. It is shown that the severe acceleration does influence this parameter in a manner consistent with the hypothesis of a reverse transition. 
In Fig. 24, the variation of the $R_{\theta}$ with axial position (according to predictions from turbulent boundary-layer calculations) is presented for a few of the tests of Fig. 18. Note especially that even for the lower stagnation pressures, the minimum $R_{\theta}$ is considerably above values at which forward transitions are customarily observed for lower pressure-gradient flows such as over flat plates and in pipes. The heat-transfer coefficient distributions obtained by Kolozsi (57) for two different stagnation conditions are illustrated in Fig. 25, as is the nozzle contour used. The wall temperatures were maintained nearly constant throughout the nozzle, such that the ratio $T_{w} / T_{0}$ remained between 0.50 and 0.55 at all stations. Thus, the variable properties correction based upon the arithmetic mean temperature (i.e., in Eq. 50) was a maximum of $+20 \%$ at the nozzle inlet and about $+10 \%$ at the throat. Note that the throat heattransfer coefficient for the higher stagnation-pressure test was about $65 \%$ of that predicted by the closedform approximation (Eq. 50), with the variable properties correction noted. This comparative result is quite close to that obtained by Back, Massier, and Gier (59), illustrated in Fig. 20 for approximately similar stagnation conditions. The data of Kolozsi have also been compared with type B and D boundarylayer predictions (see Fig. 19 for designations) in Fig. 25. The data appear to fit the two types of predictions without much preference in the subsonic region. In the throat and supersonic region, the data compare preferentially and quite reasonably with curve D: Note the rapid decrease beyond the throat of both the data and the predictions. Because of the small divergence angle, a relatively larger growth of the thermal boundary layer results compared with a wider-angle expansion region and a predicted more rapid decline of the heat-transfer coefficient. The comparatively high value of the heat-transfer coefficients measured in the entrance region is readily explained by the fact that a fine screen was placed at the entrance of the nozzle to disrupt the existing boundary layer in the nozzle approach passage, thus creating a new boundary layer beginning at the entrance to the first segment of the nozzle (57). The boundary-layer calculation was made with necessarily finite (but arbitrary) entrance values of $\phi$ and $\theta$ of 0.001 and 0.005 inches, respectively. The resulting predicted behavior of $h_{g}$ follows that in the nozzleinlet region reasonably well but would be better if thinner boundary layers had been assumed for the initial condition.

The data of Fortini and Ehlers reported in Reference 58 were restricted to essentially one nominal set of operating conditions $-300 \mathrm{psi}$ stagnation pressure and $1600^{\circ} \mathrm{R}$ stagnation temperature. Of the tests reported, three were made at the nominal conditions, with no flow obstruction upstream of the nozzle. The average of these data is presented in Fig. 26 where they are compared with predictions of the closed-form equation (Eq. 48), and to results of turbulent boundary-layer calculations as derived in 
Section II A, with $n=0$. Since rather large deviations between the measured mass-flux and the onedimensional-flow distribution were observed, the experimental distribution was used in making both the closed-form approximation and boundary-layer heat-transfer predictions. (It was found that the measured mass-flux distribution agreed very well with predictions from axially symmetric method-of-characteristics calculations in the supersonic region.) As is evident, the agreement between the data and one of the turbulent boundary-layer predictions $(n=0$, film properties curve $\mathrm{B})$ is quite reasonable out to very high area ratios. Unfortunately, the few data obtained in the throat region were somewhat more scattered than elsewhere in the nozzle. Some difficulty was encountered with spurious readings from two thermocouple plugs. The arbitrarily drawn average curve presented in the original Reference 58 is omitted because of its uncertainty in the throat region. One could conclude, however, that the most probable value of Fortini and Ehlers' throat heat-transfer coefficient lies between 70 and $85 \%$ of the closed-form equation (Eq. 50) prediction. In comparison, the data of Back, Massier, and Gier (59) at the throat for their closest test conditions $\left(254 \mathrm{psi}, 1500^{\circ} \mathrm{R}\right)$ were about $70 \%$ of the comparable closed-form equation prediction (Fig. 20). Over most of the expansion region, the data of Fortini and Ehlers closely follow the boundary-layer prediction based upon film-temperature properties evaluation and $n=0$, curve B, whereas the data of Fig. 20 tend to fall 5 to $15 \%$ below the comparable prediction, consistent with the throat data comparison. In the contraction region, both the limited data of Fig. 26 and the average of the data of Figs. 19 and 20 follow the closely similar curve B and curve D predictions satisfactorily without much preference.

Some data were also obtained by Fortini and Ehlers (58) with significant flow disturbance upstream of and within the nozzle contraction region. These data shed some light on the deviations from the steady, uniform-flow heat transfer which may result from combustion-initiated large-scale turbulence or secondary flows to be expected in a rocket nozzle, or from reactor-core-initiated flow disturbances in a nuclear rocket nozzle. In their tests, a simulated reactor core (a plug with many holes) mounted just upstream of the nozzle inlet resulted in about $25 \%$ higher throat heat flux than was obtained in the tests with undisturbed approach flow. A V-gutter turbulence generator mounted right in the contraction region of the nozzle (up to an area ratio of 10 ) resulted in about $55 \%$ higher throat heat flux.

\section{E. Effect of Acceleration on Turbulence}

In order to gain some insight into the mechanism responsible for the reduction of heat transfer below that anticipated for a fully turbulent boundary layer observed at low stagnation pressures, Back (59) 
has considered the boundary-layer turbulence energy equation (e.g., Reference 63). Using the conventional notation of Reference 63, Back wrote the equation for the convection of turbulent kinetic energy by the mean flow as

$$
u_{j} \frac{\partial \frac{q^{2}}{2}}{\partial x_{j}}=-\overline{u_{i}^{\prime} u_{j}^{\prime}} \frac{\partial u_{i}}{\partial x_{j}}-\frac{\partial}{\partial x_{j}} u_{j}^{\prime}\left(\frac{p^{\prime}}{\rho}+\frac{q^{2}}{2}\right)+\nu_{u_{i}^{\prime}} \frac{\partial^{2} u_{i}^{\prime}}{\partial x_{i}^{2}}
$$

where the first term on the right-hand side of the equation represents the production of turbulent kinetic energy by the working of the mean velocity gradients against the Reynolds stresses. The second term represents work done by the turbulence against the fluctuation pressure gradients, and the third term the convection of turbulent kinetic energy by the turbulence itself. Finally, the last term represents the transfer of energy by the turbulent viscous stresses. For a two-dimensional flow with a pressure gradient, the first term can be resolved into its significant parts, representing the production (or decay) of convected turbulent kinetic energy.

$$
\left.\overline{-u_{i}^{\prime} u_{j}^{\prime}} \frac{\partial u_{i}}{\partial x_{j}}=-\overline{u^{\prime} v^{\prime}} \frac{\partial u}{\partial y}-\overline{\left(u^{\prime 2}\right.}-\overline{v^{\prime 2}}\right) \frac{\partial u}{\partial x}
$$

The remaining terms on the right-hand side of Eq. (84) depend on the turbulence produced. The first term in Eq. (85) is always positive and leads to a production of turbulent kinetic energy. For more usual flow with negligible streamwise flow acceleration, it is clear that the second term of Eq. (85) can be neglected. However, for flow with acceleration, the second term leads to a decay of turbulence, provided $u^{\prime 2}>v^{\prime 2}$. Thus, a measure of the importance of flow acceleration in reducing the net production of turbulent kinetic energy is given by the ratio of the two terms; i.e.,

$$
\chi=\frac{\left.\overline{\left(u^{\prime 2}\right.}-\overline{v^{\prime 2}}\right) \frac{\partial u}{\partial x}}{-\overline{u^{\prime} v^{\prime}} \frac{\partial u}{\partial y}}
$$


To establish a variation of $\chi$ in the streamwise direction requires a knowledge of the turbulent quantities across the boundary layer. In the absence of direct turbulence measurements in an accelerating flow, Back (59) has adapted flat-plate measurements of Klebanoff (64) at a momentum-thickness Reynolds number of about $8 \times 10^{3}$, which yields an average value of $\left(\overline{u^{\prime 2}}-\overline{v^{\prime 2}}\right) /\left(-u^{\prime} v^{\prime}\right) \approx 1.8$. This ratio is believed not to vary appreciably across most of the boundary layer. The velocity gradient $\partial u / \partial y$ was taken from the law of the wall to be $(2.5 / 30)\left(\tau_{w} / \rho \nu\right)$. The streamwise velocity gradient was approximated as being equal to the free-stream value $d U / d x$, finally giving

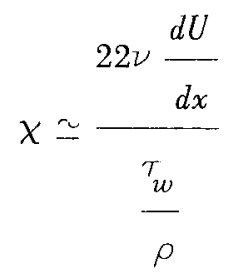

with the proportionality constant obviously being arbitrary. The variation of the parameter $\chi$ along the nozzle, the contour of which is shown in Fig. 14, was computed and is presented in Fig. 27 for one stagnation temperature and a range of stagnation pressures. With decreasing stagnation pressures, the increasing values of $\chi$ characterize the predicted reduction in net production of turbulent kinetic energy. Although the development of this parameter and the arguments behind it are somewhat less than rigorously adaptable to the problem at hand, the variation of $\chi$ along the nozzle does display a trend of being largest in the convergent section before diminishing through the throat and divergent section, which is consistent with the observations of reduced heat transfer at low stagnation pressures. Detailed turbulence measurements in strongly accelerated flows would be of considerable value in assessing the validity of the proposed reverse transition mechanism and its influences. 


\section{ROCKET THRUST-CHAMBER MEASUREMENTS}

\section{A. Literafure}

As discussed in the Introduction of this Chapter, the rocket thrust chamber has not been a very satisfactory research apparatus for investigating the rocket thrust-chamber heat-transfer problem. The numerous competing, nonrepetitive, complicating factors encountered therein which influence heat transfer make it extremely difficult to clarify the picture sufficiently to permit one to obtain results of general validity. Nevertheless, the final test of the utility of analytical and more basic experimental investigations must be the comparison of predictions resulting from these investigations with actual rocket thrustchamber heat fluxes. Unfortunately, the only meaningful comparisons are those with local heat-flux data or, at least, with data which are circumferentially averaged over a short axial length. Such data are obtained only from thrust chambers specially instrumented for this purpose, as described in Section B. Most engine development programs have not included such tests. Consequently, the limited data available are from applied research investigations at thrust levels small compared with those of the engines now under development for boosters. In 1958, Rose (65) made semilocal calorimetric measurements in a small thrust chamber operating with nitric acid-ammonia propellants and summarized results of similar data available from other investigations up to that date. In 1960, Neu (66), reported experimental data from liquid oxygen - heptane rocket thrust-chamber tests at small thrust. This particular propellant combination, widely in use for large booster engines, has the unique characteristic of causing deposits of carbon to be rapidly laid down on the thrust-chamber walls. Although this adds still another significant unpredictable complicating factor, the result is a dramatic reduction in heat flux, serving to relieve the engine cooling problem. The question of the prediction of the cause and effect of this deposition has been discussed by Sellars (67). More recently, local rocket thrust-chamber data useful for comparisons with predictions have been obtained over a wide range of conditions with nitrogen tetroxide - hydrazine propellants by Welsh and Witte (52) and by Witte and Harper (68). Powell (69 and 70) has gathered local heat-flux data with the higher-energy propellants chlorine trifluoride - hydrazine and liquid oxygen-liquid hydrogen at a thrust level of about $5000 \mathrm{lb}$. Rupe (71) has obtained data at a thrust level of about $20,000 \mathrm{lb}$ and has shown dramatic local variations in chamber heat fluxes due to injection mass distributions and the influence of unstable combustion. 


\section{B. Experimental Techniques}

The operating rocket thrust chamber as a heat-transfer device has three unique characteristics: (1) very rapid establishment of steady flow, (2) very high heat fluxes, and (3) very sharp axial (and sometimes circumferential) gradients of heat flux. The third characteristic establishes a requirement for very localized measurements, whereas the first two characteristics suggest methods of making such measurements. The extremely high heat flux establishes the requirement for considerable cooling if the thrust chamber is to operate for any appreciable length of time (i.e., more than a few seconds). Because the fluxes are generally so high, it is possible to divide the thrust chamber into numerous axially short segment lengths which are individually cooled. An example of a thrust chamber designed for such steadystate calorimetric measurements, as reported in References 68 and 69 , is illustrated in Fig. 28 . By regulating the flow to each of the passages, it is possible to equalize the temperature rise in each passage so as to minimize passage-to-passage heat transfer by coolant convection to the barrier walls. The error introduced by axial conduction in the wall between the gas and the coolant can be made acceptably low by thinning the wall out to about $10 \%$ of the passage axial length, as illustrated in Fig. 28. Although the number of passages shown in the Figure are sufficient to permit determination of axial variations in heat flux with adequate resolution, the principal drawback of this technique is that it cannot resolve circumferential variations. Such variations can become significant with certain types of propellant injectors and certain operating conditions to be discussed in Section C. The most frequently used coolant for calorimetric measurements such as these is water, generally metered by turbine meters and temperature-measured with differentially wired thermocouples.

The very rapid establishment of full flow conditions in rocket thrust chambers (i.e., typically much less than $1 \mathrm{sec}$ ) and the currently available capability of fully measuring all necessary performance parameters in a few seconds of steady flow operation have led to the practice of building most thrust chambers of heavy uncooled metal walls for initial developmental testing of the combustion characteristics. This practice naturally suggested a transient heating measurement of local heat flux, in which time temperature histories of local areas of the wall are recorded during the short test duration. In principle, it is then possible to feed this time-temperature history into a computer programmed to solve the transient heat-conduction equations for the configuration of interest to determine what unique heat-flux input was required to produce the experimentally recorded history. Analytical investigations of this problem (72) have shown that such a computation can be made to yield adequately accurate heat-flux 
results by solving the one-dimensional (radial) heat-flux equation in cylindrical coordinates with two experimentally measured time - temperature boundary conditions, using appropriately temperature-variable thermal properties of the wall. The analysis also demonstrated the necessity of getting one of the thermocouple junctions as close to the combustion surface of the wall as possible and further dictated the necessary temperature sampling rate. An example of one configuration of the thermocouple plug utilized by Powell and Price (72) is illustrated in Fig. 29. The question of neglecting the axial variation of heat flux in solving the transient conduction equation was investigated analytically and experimentally by comparing measured heat flux distributions in identically contoured calorimetric and transient pluginstrumented nozzles operating under closely similar combustion conditions. This comparison, in which agreement within about the $\pm 10 \%$ circumferential variations of the local data was demonstrated, is reported in Reference 72 , as are further details of the transient conduction program, the plug construction techniques, and the data-sampling requirements. The most significant advantages of this method of heatflux determination are the possibility of making pointwise (i.e., truly local) measurements and the adaptability to inexpensive thrust chambers and short test duration.

Although a comprehensive discussion of propellant injection and combustion principles is beyond the scope of this Chapter, several representative injector types are illustrated in Figs 28 and 30 . A brief qualitative description of their characteristics will be of use as background to the discussion of heat transfer in the combustion chamber. The injector illustrated in Fig. 30(a) is known as an enzian or splash-plate injector. By directing the propellant jets to impinge behind the splash plate, a tightly confined initial combustion is established, with a strong recirculation of hot gases behind the plate. This recirculation action probably tends to obliterate the discrete nature of the combustion gas flow that would otherwise result from the use of a limited number of pairs of propellant jets. It also results in a rather short flow length, in which the first $90 \%$ of the final total temperature is achieved. As the partially combusted gases flow through the aperture of the splash plate, a separated region is created which, because of the vortex shedding, is characterized by a strong reverse flow near the wall. Figure 30 (b) shows a showerhead-type injector comprised of a large number of axially directed propellant jets. Because there is no forced mixing due to impingement or recirculation (both of which are provided in the enzian injector), reaction results only by jet breakup, followed by radial diffusion of the droplets and evaporation and diffusion of the propellant due to heat release from the initial small amount of random mixing and combustion. Because of the nature of the process, it is almost mandatory that the number of jets be kept very large in order to keep the mixing scale small with respect to chamber dimensions. 
The dependence on the relatively slow diffusional process causes the approach to the final total temperature to be rather gradual. The type of injector most prevalent in very large thrust-chamber injectors also utilizes a very large number of jets and usually has some impingement of adjacent propellant jets provided by drilling the jets at an angle to the axis. However, there is no organized recirculation of part of the hot gases such as results from the splash plate of the enzian injector. The energy release patterns of this type of injector are probably about midway between the enzian and showerhead injectors. The injector illustrated in Fig. 28 typifies the use of impinging jets in small thrust injectors, where, because of lower limits on the size of practically drillable jet orifices, it becomes necessary to use only a limited number. This results in spatially nonuniform mass-injection distributions. The nonuniformities have been shown by Rupe (7I) to result in substantial circumferential gradients in heat flux, which, surprisingly, persist all the way through the nozzle. A wide variety of other injectors is in use, each with its own peculiar flow and energy-release patterns. As will be shown, these peculiarities of the various injectors directly influence the heat-flux distribution.

\section{Combustion-Chamber Heat Flux}

Because of the general lack of specific quantitative knowledge of the heat-release patterns of injectors, the usual practice in design analyses of the cooling of rocket thrust chambers is to assume that the combustion chamber heat flux is constant at the value calculated for the nozzle entrance. If the closed-form approximation (Eq. 50) is used, this is equivalent to assuming a boundary-layer thickness at the nozzle entrance equivalent to that which would be expected at the end of a constant-area duct about equal in length to the nozzle (see Fig. 10, case 2a). An example of a measured chamber heat-flux distribution which agrees closely with such a prediction is shown in Fig. 31, where the enthalpy driving potential specified by Eq. 82 has been employed. Since driving potentials are not directly measured or known, all results from rocket thrust-chamber tests are presented as direct heat-flux rather than heat-transfer coefficients as were used for the air results. The propellants used in the tests of Fig. 31 were nitrogen tetroxide-hydrazine. 'The contraction-area ratio was $4: 1$, which is typical for thrust chambers of a few thousand pounds. (Thrust chambers designed to operate at smaller thrust levels generally tend to have higher contraction ratios because of the difficulty in building a very small-diameter injector. On the other hand, thrust chambers designed to operate at larger thrust levels generally tend to have lower contraction ratios in order to reduce weight.) Note that the heat flux in the segment of the chamber adjacent to the injector is essentially up to the full value of the heat flux in the remainder of the chamber 
and that at the nozzle-entrance region. This is undoubtedly the result of the very rapid initial rise in total temperature and the strong reverse flow pattern, both characteristic of the enzian injector.

Unfortunately, agreement between predictions and measured heat fluxes in combustion chambers such as that illustrated in Fig. 31 is not typical. In particular, in a thrust chamber with a large contraction ratio (like the one for which data are presented in Fig. 32 for the same propellants and injector as in Fig. 31), it is observed that the measured heat flux in the chamber is nearly $100 \%$ above the prediction. While there is no known quantitative relationship, qualitatively, this pattem of high heat flux (relative to prediction) is generally observed for high contraction-ratio chambers for a variety of propellants and injector types. This is probably due to the fact that local combustion-dominated flows (such as the recirculation for the enzian injector) can have velocities far in excess of the cross-sectionally averaged velocity which is utilized in making the predictions.

The conclusion should not be hastily drawn that the contraction ratio is sufficient to correlate heat flux in a combustion chamber, inasmuch as data obtained with the same propellants and injector but with a thrust chamber having a contraction ratio of only 1.64 to $l$ (presented in Fig. 33) also show dramatic differences between predictions and experimental observations. In this case, the average flow velocities are so high that the residence time of the reacting propellants is considerably reduced. Thus, local effects due to chemical reaction (i.e., combustion) appear to persist all the way to the throat. There is no good detailed mechanistic argument readily available that would quantitatively predict or explain this influence on heat flux. Elevated chamber and contraction-region (even throat-region) heat fluxes have been frequently (but not necessarily typically) observed with low contraction ratio thrust chambers.

Figure 34 shows a chamber heat-flux distribution measured with an enzian ejector (see Fig. 30a) compared with that of a showerhead injector (see Fig. 30 b) operating at similar conditions with the same propellants and thrust chamber. Note the gradual rise in heat flux from the injector to the nozzle entrance, where the values reach a level quite close to that predicted by the closed-form approximation (Eq. 50). This pattern is quite consistent with the characteristics of a showerhead injector (described in Section IV B), i.e., a very gradual heat-release pattern and a very small scale of flow nonuniformities. It is probable that no significant organized combustion-dominated flows departing from the average flow are present. In view of the high fraction of theoretical performance $\left(c^{*}\right)$ and low chamber heat flux exhibited by this injector, it is fair to ask why other types such as the enzian should be used. One reason is that the number 
of injection orifices tends to run into the many thousands when the injector is scaled up to large thrusts. Thus, the cost and reproducibility of flow from jet to jet tend to become unacceptable. But more serious is the fact that the gradual heat-release pattern which promotes low heat fluxes also makes large operating thrust chambers more susceptible to severe combustion pressure oscillations. The severe oscillations are believed to be due to periodic disturbances moving through regions of mixed - but only partially reacted - propellant gases, with sudden energy release occurring as the disturbance passes. This wave process is reinforced and becomes steeper to the point at which sharp-fronted waves with pressure ratios greater than 10 to 1 have been observed, causing some combustion research investigators to believe that continuously spinning detonation waves are established. Such waves are very damaging, principally because they tend to increase wall heat fluxes sharply beyond normal engine cooling capabilities, with spectacular failures resulting.

Examples of the range of influences of nonlinear combustion oscillations on chamber heat fluxes are illustrated in Fig. 35(a), in which the ratio of observed heat flux to that predicted by Eq. (50) is plotted versus distance from the injector face, multiplied by the contraction ratio. (By use of this modification of the length coordinate, the abscissa values become proportional to the average residence time of the propellant within the thrust chamber.) It is significant to note that present understanding of the causes and effects of nonlinear combustion pressure oscillations is insufficient to predict whether or not they will occur and, if so, what their influence will be on temporal or spatial distributions of heat flux. Some basic experiments have been made to determine the influence of oscillating pressures on heat transfer $(3,4,5)$. Unfortunately, such experiments were made with isentropic waves of amplitude comparable to the mean pressure and not with nonisentropic waves of amplitude many times the mean pressure such as those believed to have been encountered by Rupe (71).

In addition, Fig. 35(a), illustrates some of the circumferential variations in heat flux (indicated by range covered by arrows) that result from the mass-flux pattern established by the injector. By taking point measurements with tran sient temperature plugs in a fairly large thrust engine $(20,000 \mathrm{lb})$ with a relatively coarse injector pattern, Rupe (7I) was able to resolve experimentally heat-flux variations of nearly 10 to 1 (near the injector), depending on local position with respect to an individual injectororifice pair. On some scale, relative to the injector-element scale, variations such as these probably occur within all thrust chambers.

Figure 35 also shows data obtained with two high-energy propellant combinations - chlorine trifluoride-hydrazine and liquid oxygen-liquid hydrogen. The special feature of combustion of these 
propellants is the fact that a substantial fraction of the energy released by combustion can become tied up in dissociating species such as hydrogen molecules to hydrogen atoms and water into hydrogen and hydroxyl radicals (see Section II E). The limit of the effect of the recombination of these species in the cool boundary layer can be accounted for approximately by assuming equilibrium recombination and adopting the enthalpy driving-potential concept, the details of which are specified in Reference 6 and discussed in Section II E. This has been done in calculating the predictions to which the experimental data of Figs. $31-35$ have been compared. It was found, however, that the percentage increases due to the possibility of equilibrium recombination are too small in these tests to be verified by comparisons with chamber heat-flux data because of the wide variety and magnitude of competing unpredictable influences of the combustion. In fact, the principal point to be established by the data and the discussion of this Section is that the reliable prediction of heat flux in a combustion chamber is not possible because of the inadequate status of quantitative knowledge of rocket-engine combustion phenomena. Until sufficient quantitative knowledge is gained, attempts at refined chamber heat-transfer calculations are not justified. The limit of useful analysis is probably calculations from some simple correlation equation such as Eq. (50), modified by qualitative and intuitive arguments about the combustion flow as characterized by the injector, propellants, and operating conditions of interest.

\section{Nozzle Heat Flux}

Because of the severe acceleration that occurs in the nozzle, and the increasing distance from the injector, it might be argued qualitatively that the direct influences of combustion such as secondary flows and heat-release distribution should begin to decay as the acceleration commences at the nozzle entrance, and should be largely decayed by the point of maximum acceleration, the throat. This qualitative expectation is supported to some extent by the experimental nozzle heat-flux distributions from Reference 52 , presented in Figs. 31, 32, and 33 of this Chapter. Even though there are considerable qualitative differences in the heat-flux distributions in the contraction region and the region just ahead of the geometrical throat, the distributions beyond the throat all compare quantitatively quite well with the prediction based upon the closed-form approximation equation (Eq. 50). In the expansion region (out to the limited area ratio utilized in the tests of Reference 52 about 3.5 ), the experimentally measured heat fluxes all fall within a band from 80 to $100 \%$ of the value predicted by Eq. (50) at corresponding area ratios and most within an even tighter band, i.e., $85-90 \%$ of the prediction. 
Although plots such as those of Figs 31,32 , and 33 are useful for conveying an idea of the distributions and numerical values of the heat flux, they are not very satisfactory for generalizing the results or for ready comparisons with predictions over a range of conditions. For this purpose, it is appropriate to turn back to the dimensionless parameter presentation such as that used to correlate the data from the air experiments. In particular, the parameter $C_{h} P_{r}^{0.6}$ is plotted versus $R_{D}$ for the reasons described in Section III D-3. As in the case of the air data, these parameters are computed on the basis of evaluating the properties at the free-stream static temperature. In order to reduce the experimental heat-flux values to heat-transfer coefficients, computations of the driving potential were made on the enthalpy-difference basis $\left(I_{0}-I_{f, w}\right)$ described in Section II E. Account was taken of actual reductions in performance below the theoretical value, but it was assumed that the combustion process was completed by the time the flow entered the nozzle. For comparison with these data, presented in Figs. 36, 37, 38 and 39, lines are drawn corresponding to (1) the predictions from Eq. (50) (based on arithmetic mean temperature evaluation of properties) for a value of $T_{w} / T_{0}$ typifying the data of the particular Figure, and (2) the predictions from Eq. (48), with $T_{\text {ref }}$ selected as the adiabatic wall temperature $T_{a w}$.

Attention is first called to the part of each Figure corresponding to the highest subsonic area ratio for which data are reported for each of the four different contraction-area-ratio nozzles. They have in common the fact that the slopes of the curves drawn through the experimental data of all four of these nozzles is quite close to that of the prediction equation; i.e., $C_{h} P_{r}^{0.6} \sim R_{D}^{-0.2}$. For all but the 2.5:1. nozzle (Fig. 37), the data exceed the predicted values. The experimental values for the highest subsonicarea-ratio plot for the 8:1 area-ratio nozzle lie considerably above the predicted line for reasons discussed in Section IV C. Notice, however, that as the flow proceeds to lower subsonic area ratios, the lower $R_{D}$ (i.e., lower total pressure) data begin to drop off considerably with respect to the prediction lines. As the $R_{D}$ increases, values of $C_{h} P_{r}{ }^{0.6}$ rise abruptly relative to the prediction line. At still larger $R_{D}$, they begin to fall off gradually with increasing $R_{D}$ in most cases reaching what appears to be the start of a -0.2-power dependence region. Note that there is evidence of this behavior out to even the highest supersonic-area ratios. This behavior is significantly similar to that exhibited by the data from the hotair tests in Fig. 24. Perhaps this results from the same mechanism proposed to explain the behavior of the air data; i.e., at lower $R_{D}$ (lower stagnation pressures) turbulent boundary layers entering the nozzle are worked upon by the severe acceleration, decaying the turbulence near the wall by some turbulence decay mechanism (such as discussed in Section III E), resulting in a reduction in the wall gradients of the velocity and temperature back toward those characteristic of transitional flow. At higher $R_{D}$, i.e., 
higher stagnation pressure, the turbulence decay mechanism is not as strong (as shown in Fig. 27). Thus, at higher $R_{D}$, the acceleration does not substantially reduce the wall gradients and, hence, the heat flux. It is granted that this argument and proposed mechanism are speculative, being based on limited data, all obtained with the same propellants and injector. Injector performance and flow characteristics are known to be typically quite flow-rate sensitive and hence, sensitive to stagnation pressure. The principal reason for suggesting the mechanism at all is the remarkable qualitative similarity between these rocket-nozzle results and the air results presented in Fig. 23, and the data obtained by Back (62) with accelerated lowspeed wind-tunnel flow. Unfortunately, there are no data available from other rocket tests which cover a sufficient range of operating conditions such that a useful comparison could be made.

In order to illustrate how the heat-transfer coefficient varies at higher area ratios (and Mach numbers), typical data of Reference 68 are plotted in Fig. 40 and compared with boundary-layer predictions (Eq. A-8) as well as the closed-form approximations (Eq. 50 with $T_{\text {ref }}=T_{a m}$ and Eq. 48 with $T_{\text {ref }}=$ $\left.T_{a w}\right)$. For each of the boundary-layer heat-transfer predictions, the initial boundary-layer thicknesses at the nozzle inlet were selected so as to yield a heat flux equal to the average of the experimental data in the entrance region. The test selected at $300 \mathrm{psi}$ stagnation pressure is at sufficiently high $R_{D}$ to preclude behavior of the type discussed in the previous paragraph. For a conical expansion section such as that used in the test from which these data were obtained, the deviations of $\rho U$ from $\rho_{1} U_{1}$ are negligible except very near the throat. To facilitate the comparisons, the data have also been normalized by dividing by the local $h_{g}$ value predicted by Eq. (50) in the lower part of the Figure, as have the other predictions. Except for a few passages believed to have had erratic thermocouples, the data follow a more or less smooth decline from about $25 \%$ above the type-C predictions just beyond the throat to very close agreement with the type-C or -D predictions at high area ratios. This behavior is closely similar to results of other high area-ratio data from rocket tests at lower stagnation pressures presented in Reference 68. It is al so reasonably similar to that of the air data of Figs. 20 and 23 over the limited range of supersonic-area ratios at which data were obtained. The convergence of the high Mach-number data toward the type-C or -D boundary-layer prediction suggested the possible value of a closed-form approximation to this prediction. This was found to be readily possible over the expansion region of the nozzle (where the boundary-layer growth has become regular) by adopting $T_{a w}$ as $T_{\text {ref }}$ in evaluating $h_{g}$ from Eq. 48 (see Section II). As is evident from the bottom portion of Fig. 40, the agreement with the type-C and -D boundary-layer $h_{g}$ prediction over this portion of the nozzle is excellent.

In the contraction and throat regions, the air data and the rock et data behave quite differently, 
the air data being equal to or below the type-C boundary-layer prediction and the rocket data exceeding the prediction by as much as $40 \%$. If one were forced to speculate about a mechanism that would account for this situation, one might postulate that in the rocket nozzle flow there is superimposed upon the normal convective processes an added process, such as free-stream turbulence, which originates in the combustion chamber and which decays in the streamwise direction. It is well known that even for rocket engines operating without organized combustion pressure oscillations, a so-called combustion noise is frequently present having pressure oscillation amplitudes of several percent of the mean pressure without regular or dominant frequencies. (These free-stream flow fluctuations are probably related to free-stream turbulence phenomena.) Combustion noise of this character was usually encountered in tests in which the rocket data discussed in this Section were obtained. Although these noise-like pressure oscillations are almost always monitored only by transducers back near the injector, it is very likely that the magnitude of the oscillations decays in the flow direction. Although neither the status of theory nor basic experiments on the influence of free-stream turbulence on heat transfer $(75,76,77)$ are sufficiently well advanced to make an authoritative prediction of the influence of the free-stream turbulence, the $40 \%$ elevation of the rocket data above the air data is not inconsistent with influences of free-stream turbulence observed by others (76). Because of the large, irregular influences on heat transfer in the contraction region believed possible due to combustion-dominated secondary flows, one cannot readily differentiate between such effects and those of the free-stream turbulence in this region.

The rocket data of Fig. 40 clearly show an asymptotic preference toward predictions made on the basis of evaluating properties at the adiabatic wall temperature, supporting the arguments and evidence presented in Section II D. Unfortunately, the air data of Reference 59, which also tend to favor the same prediction basis, were restricted to low area ratios, where other factors at work make conclusive comparison difficult. The air data presented in Fig. 26 (from Reference 58) were obtained out to high expansion ratios and show a decided preference for predictions based on the film-temperature property evaluation. There is no explanation obvious to this author for the distinct difference between these particular air data and the rocket data at high area ratios. Thus, the variable properties question is not resolved by these data.

Powell $(69,70)$ has measured the distribution of local heat transfer in rocket engines operating with propellants of sufficient energy content to make considerations of the effects of recombination on heat transfer (see Section II E) potentially important. Unfortunately, the fraction of theoretical performance achieved in these investigations thus far $\left(90-95 \% c^{*}\right)$ has not been sufficient to cause predicted chemical 
recombination enhancement of heat transfer to exceed about $10 \%$, which is not readily discernible within the experimental scatter and the other influences on heat transfer. Consequently, the measured heattransfer distributions of References 69 and 70 are quite similar to those of Figs. 31, 32, and 33 with respect to predicted values.

Although this Chapter has been restricted to the problems of heat transfer from the hot gases to the cooled walls of nozzles and chambers, the reader should also be aware of the interesting and often design-limiting problems associated with the wall cooling. In the particular case of cooling with hydrogen, the two problems become closely coupled and must be solved simultaneously. Correlations of the heattransfer coefficients of hydrogen as they apply to the rocket-engine cooling problem have been discussed by Benser and Graham (78). In the case of cooling with subcooled liquids, it is frequently advantageous to make use of nucleate boiling. As discussed in Reference 79 , this regime of operation is characterized by a fixed and easily determined wall temperature, thus decoupling the problems to a large extent. 


\section{CONCLUDING REMARKS}

As should be clear to the reader from the evidence presented in this Chapter, the problem of heat transfer from hot gases to the cooled walls of nozzles is not yet "solved" in the sense of producing a theory or an empirical correlation universally accurate for all the flows of interest. Nevertheless, the analyses and experiments that have been discussed have accomplished two things: (1) They have suggested the specific basic physical processes that must be understood quantitatively before the problem can be solved and (2) they have shown how far existing methods of analysis may be in error under the limited conditions thus far investigated thereby establishing some basis for corrections to these analyses that will permit an acceptable prediction of the heat transfer for most requirements. The delineation of the processes that must be understood to "solve" the problem and the specification of procedures recommended for nozzle heat-transfer predictions at this time conclude this Chapter.

Of basic importance to all turbulent boundary-layer flows are two problems brought into sharp focus by the cooled-nozzle heat-transfer problem. First, the question of the influence of variable properties on heat transfer to severely cooled walls must be settled by definitive experiments in which local conditions, including velocity and temperature profiles, are measured over a range of velocities extending into the supersonic region and over a range of stagnation pressures. Second, the influence on heat transfer of both free-stream turbulence and gross disturbances to the boundary layer by secondary flows must be investigated and understood to such an extent that their effects can be predicted with reasonable assurance from known or specified initial conditions.

The presence of a severe favorable pressure gradient, of the magnitude peculiar to nozzle flows, adds another potentially significant, yet largely uninvestigated, basic dimension to boundary-layer flow. In particular, direct shear measurements are required to determine whether or not it is reasonable to assume that for the same local momentum-thickness Reynolds number, the skin friction of an accelerating turbulent boundary layer is the same as without a pressure gradient. The effect of strong favorable pressure gradients on turbulence production and decay within the boundary layer must be understood and somehow correlated to make the occurrence of both forward and reverse transition predictable. Some means of correlating skin friction in the transition region must also be found. For flows with strong favorable pressure gradients, where, as indicated by predictions, the thermal boundary layer can become substantially thicker than the velocity boundary layer, it is necessary to find either (1) some modified 
Reynolds analogy by gaining new insight into the turbulent form of the boundary-layer equations with pressure-gradient terms retained or (2) some general correlation of the Stanton number in terms of the local energy thickness irrespective of past history of the boundary layer. Finally, fluid mechanics and thermodynamics of the rocket combustion process must become quantitatively understood and made both predictable and describable on an instantaneous, local basis in order to make significant improvement in the prediction of the heat flux in the combustion chamber and contraction region.

As for recommended procedures for making predictions of rocket-nozzle heat transfer, it is probably worth the effort to employ one of the iterative boundary-layer solutions of the type described in Section II A for radically new nozzle configurations or peculiar entrance or operating conditions for which such solutions are not available. For such calculations, one should attempt to account for deviations from one-dimensional flow by method-of-characteristics calculations in the supersonic region and use of Eq. (83) in the transonic region. For further predictions for only slightly different configurations or conditions, especially when an answer is required in a hurry, the closed-form approximation equations can be used with reasonable precision by adjusting the constant $C$ to fit the previously obtained boundary-layer results. The question remains, however, as to which boundary-layer calculation method and which closedform approximation should be used and how the se computed results might relate to the heat fluxes to be anticipated in the rocket nozzle. Since data presented and discussed in this Chapter failed to substantiate conclusively either of the variable properties correction methods described, the choice on this critical question remains arbitrary. This author tends to favor using the adiabatic wall temperature $T_{a w}$ as the reference temperature in the closed-form approximation equation (Eq. 48), or the closely equivalent procedure of utilizing the Coles $(15) C_{f}$ for an adiabatic wall in the boundary-layer calculation (Eq. A-8). The reason for this selection is threefold: (1) the pipe and flat-plate data discussed in Section II D suggesting its basic validity, (2) the agreement at high velocities with the rocket data of Fig. 40 , and (3) the way this assumption fits in with the hypothesis of decaying free-stream turbulence (Section IV D). In order to relate predictions on this basis to expected heat fluxes in a rocket nozzle, it is probably necessary to start by multiplying the values in the transonic region by a factor of about 1.3 to 1.4 and to decrease this factor gradually to unity at stations at which the Mach number is about 4. In the chamber and the contraction regions, the values require multiplication by some factor arrived at intuitively, based upon arguments about the nature of the combustion influences, as discussed in Section IV C. A factor of 1.3 to 1.8 is probably a reasonable starting range around which adjustments can be made as the combustion flow situation may demand. For combustion gases in which a substantial fraction of 
the total energy is tied up in dissociation, account should be tak en the possible recombination enhancement of heat transfer by adjustments to the driving potential, as discussed in Section II E.

\section{ACKNOWLEDGMENT}

The author is grateful to P. F. Massier and L. H. Back for their detailed review of the manuscript and their constructive suggestions. In addition, it is a pleasure to acknowledge the considerable contributions made to the rocket heat-transfer problem by the author's colleagues past and present, including L. H. Back, W. J. Colahan, D. G. Elliott, G. W. Elverum, H. L. Gier, E. Y. Harper, D. T. Harrje, P. F. Massier, M. B. Noel, W. B. Powell, T. W. Price, R. W. Rowley, J. H. Rupe, W. E. Welsh, and A. B. Witte. 


\section{APPENDIX - A}

\section{Coles' Skin-Friction Coefficient and von Kármán Form of Reynolds Analogy}

Coles (15) has shown that most of the carefully measured skin-friction coefficient data from adiabatic flow over flat plates over a wide range of $R_{\theta}$ and $M$ can be correlated to within a few percent by

a single curve of $\bar{C}_{f}$ versus $\bar{C}_{f} \bar{R}_{\bar{\theta}}$. These low-speed values of $\bar{C}_{f}$ and $\bar{R}_{\theta}$ are related to the actual $C_{f_{a}}$ and $R_{\theta}$ values by

$$
\bar{C}_{f}=\frac{\rho \mu_{s}}{\rho_{a w} \mu_{a w}} C_{f_{a}}
$$

and

$$
\overline{C_{f}} \bar{R}_{\bar{\theta}}=\frac{\rho \mu}{\rho_{a w} \mu_{a w}} C_{f_{a}} R_{\theta}
$$

where subscript aw refers to the adiabatic wall or recovery temperature and subscript $s$ refers to a sublayer temperature given by

$$
\frac{T_{s}}{T_{a w}}=1+17.2\left(\frac{T_{0}}{T_{a w}}-1\right)\left(\frac{\bar{C}}{\bar{C}}\right)^{1 / 2}-305\left(\frac{T_{0}}{T_{a w}}-\frac{T}{T_{a w}}\right)_{2}^{C_{f}}
$$

The values of $\bar{C}_{f}$ versus $\bar{C}_{f} \bar{R}_{\theta}$ utilized by Coles are plotted in Fig. 3, where they are compared with the Blasius equation. They are also tabulated in Table A-1. For values of $\bar{C} \bar{R}_{\bar{\theta}}$ above 64.8, the extrapolation curve given by

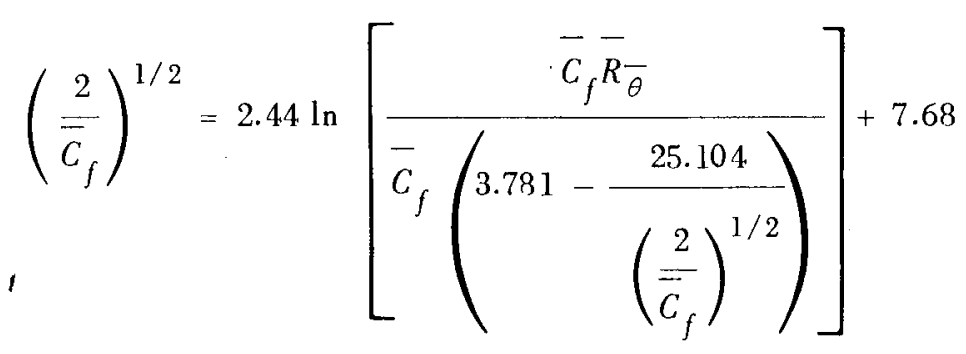


can be used. Values of $\bar{C}_{f}$ for $\bar{C}_{f} \bar{R} \bar{\theta}$ below 2.51 can be computed from

$$
\bar{C}_{f}=\frac{0.009896}{\left(\bar{C}_{f} \bar{R}_{\bar{\theta}}\right)^{0.562}}
$$

In this region, the $R_{\theta}$ are below those normally associated with turbulent flow. Equation (A-5) is used simply to get an order of magnitude value of $C_{f}$ with which to start a thin entrance-condition calculation. Utilizing a power relation for viscosity, $\mu \sim T^{m}$, Eqs. (A-1) and (A-2) can be put into a more convenient form given, respectively, by Eq. (33) and by

$$
\frac{C_{f_{a}} R_{\theta}}{\overline{C_{f} \overline{R_{\theta}}}}=\frac{1}{\left(\frac{T_{a w}}{T}\right)^{1-m}}
$$

The form of the Reynolds analogy adopted to obtain the calculation results presented in this Chapter is based on Assumption 13, that when $\phi=\theta, C_{h}$ is related to $C_{f} / 2$ by the von Kármán form of the Reynolds analogy given by Eq. (38). For $\phi=\theta$, Eq. (38) can be rewritten directly as

$$
C_{h}=\frac{\frac{C_{f}\left(R_{\phi}\right)}{2}}{1-5\left(\frac{C_{f}\left(R_{\phi}\right)}{2}\right)^{1 / 2}\left[1-P r+\ln \frac{6}{5 P r+1}\right]}
$$

where $R_{\phi}$ is the parameter $\rho U \phi / \mu$. By the intuitive reasoning and the comparison with data given in Section II A-6, Eq. (A-7) is modified for cases in which $\phi \neq \theta$ by multiplying (A-7) by $(\phi / \theta)^{n}$, giving

$$
C_{h}=\frac{\frac{C_{f}\left(R_{\phi}\right)}{2}\left(\frac{\phi}{\theta}\right)^{n}}{1-5\left(\frac{C_{f}\left(R_{\phi}\right)}{2}\right)^{1 / 2}\left[1-P r+\ln \frac{6}{5 P r+1}\right]}
$$


where the notation $C_{f}\left(R_{\phi}\right)$ denotes a value of $C_{f}$ determined either from Eq. (33) (for adiabatic wall $C_{f}$ ) or from Eq. (36) (for film properties $C_{f}$ ), $C_{f}$ having been evaluated from Table A-1, Eqs. (A-4), and (A-5), with $R_{\phi}$ replacing $R_{\theta}$.

Table A-1. Local friction relation for turbulent boundary layer at constant pressure ${ }^{1}$

\begin{tabular}{|c|c|}
\hline $\bar{C}_{f} \overline{\bar{\theta}}$ & $\bar{C}_{f}$ \\
\hline 2.51 & 0.00590 \\
3.10 & 0.00524 \\
3.97 & 0.00464 \\
4.88 & 0.00426 \\
5.73 & 0.00398 \\
7.41 & 0.00363 \\
8.94 & 0.00340 \\
12.75 & 0.00308 \\
16.36 & 0.00290 \\
23.2 & 0.00269 \\
29.6 & 0.00255 \\
35.9 & 0.00246 \\
41.8 & 0.00238 \\
53.6 & 0.00227 \\
64.8 & 0.00219 \\
\hline
\end{tabular}


APPENDIX - B

\section{Boundary-Layer Shape Parameter Evaluation}

In order to calculate the boundary-layer shape parameters $\delta^{*} / \theta, \theta / \delta, \phi / \Delta$, etc., it is necessary to specify velocity distributions over the velocity boundary-layer thickness $\delta$ and temperature distributions over the temperature boundary-layer thickness $\Delta$. The distributions adopted, in accordance with Assumption 15, are

$$
\frac{\vec{u}}{U}=\left(\frac{y}{\delta}\right)^{1 / 7} \quad y \leq \delta \quad \frac{\bar{u}}{U}=1 \quad y>\delta
$$

and

$$
\frac{\overline{t_{0}}-T_{w}}{T_{0}-T_{w}}=\left(\begin{array}{c}
y \\
\Delta
\end{array}\right)^{1 / 7} \quad y \leq \Delta \quad \frac{\overline{t_{0}}-T_{w}}{T_{0}-T_{w}}=1 \quad y>\Delta
$$

By rearrangement of Eq. (B-2), making use of the isentropic relationship between static and stagnation temperature and the velocity distribution of Eq. (B-1), the local density distribution is given by

$$
\bar{t} \frac{\bar{\rho}}{\bar{T}}=a\left(1+\frac{b}{\zeta} s-c s^{2}\right) \quad y<\Delta \text { or } \delta
$$

where

$a=\frac{T_{w}}{T}, \quad b=\frac{T_{0}}{T_{w}}-1, \quad c=\frac{T_{0}-T}{T_{w}}, \quad s=\left(\frac{y}{\delta}\right)^{1 / 7}, \quad\left(\begin{array}{l}\Delta \\ \delta\end{array}\right)^{1 / 7}$

In evaluating the shape parameters, cognizance must be taken of two special cases in which Eq. (B-3) must be modified. 
Case $I:$ when $\delta \leq \Delta, \delta<y \leq \Delta, \quad \bar{t} / T=a\left(1+\frac{b}{\zeta} s-c\right)$

Case II: when $\delta>\Delta, \Delta<y \leq \delta, \quad \bar{t} / T=a\left(1+b-c s^{2}\right)$

Note that the Crocco temperature distribution is a special case of Eq. (B-3), in which $\zeta=1$, the situation for flow over a flat plate, with $\operatorname{Pr}=1$.

From the integral definition of $\theta$ for a thin boundary layer given by Eq. (12), it can be shown that

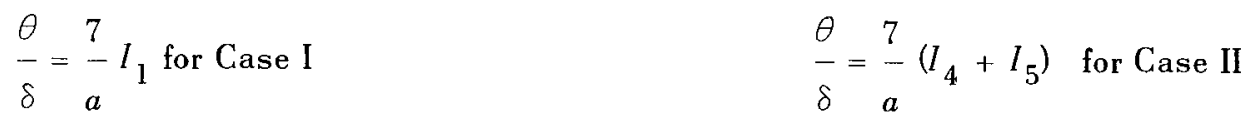

where the definite integrals $I$ are given by

$$
I_{1}=\int_{0}^{1} \frac{s^{7}(1-s)}{1+\frac{b}{\zeta} s-c s^{2}} d s, \quad I_{4}=\int_{0}^{\zeta} \frac{s^{7}(1-s)}{1+\frac{b}{\zeta} s-c s^{2}} d s, \quad I_{5}=\int_{\zeta}^{1} \frac{s^{7}(1-s)}{1+b-c s^{2}} d s
$$

Similarly, from Eq. (15),

$$
\frac{\phi}{\delta}=\frac{7 \zeta^{7}}{a}\left(\zeta I_{2}^{\prime}+I_{3}^{\prime}\right) \text { for Case I } \quad \frac{\phi}{\delta}=\frac{7 \zeta^{8}}{a} I_{1}^{\prime} \text { for Case II }
$$

where

$I_{2}^{\prime}=\int_{0}^{1 / \zeta} \frac{w^{7}(1-w)}{1+b w-c \zeta^{2} w^{2}} d w, \quad I_{3}^{\prime}=\int_{1 / \zeta}^{1} \frac{w^{6}(1-w)}{1+b w-c} d w, \quad I_{1}^{\prime}=\int_{0}^{1} \frac{w^{7}(1-w)}{1+b w-c \zeta^{2} w^{2}} d w$

From Eqs. (B-6) and (B-7),

$\zeta=\left[\frac{\phi I_{1}}{\theta\left(I_{2}^{\prime}+\frac{I_{3}^{\prime}}{\zeta}\right)}\right]^{1 / 8}$ for Case I

$\zeta=\left[\frac{\phi\left(I_{4}+I_{5}\right)}{\theta I_{1}^{\prime}}\right]^{1 / 8}$ for Case II

(B-8) 
Finally, from Equation (9),

$$
\frac{\delta^{*}}{\delta}=\zeta^{7}-\frac{7}{a}\left(I_{2}+I_{3}\right) \text { for Case I }
$$

$$
\frac{\delta^{*}}{\delta}=1-\frac{7}{a}\left(I_{6}+I_{7}\right) \text { for Case II }
$$

where

$$
\begin{array}{ll}
I_{2}=\int_{0}^{1} \frac{s^{7}}{1+\frac{b}{\zeta} s-c s^{2}} d s, & I_{3}=\int_{1}^{\zeta} \frac{s^{6}}{1+\frac{b}{\zeta} s-c} d s \\
I_{6}=\int_{0}^{\zeta} \frac{s^{7}}{1+\frac{b}{\zeta} s-c s^{2}} d s, & I_{7}=\int_{\zeta}^{1} \frac{s^{7}}{1+b-c s^{2}} d s
\end{array}
$$




\section{APPENDIX - C}

\section{Boundary-Layer Thicknesses and Integral Equations for Thick Boundary Layers}

If now the restriction is removed that the boundary-layer thicknesses $\delta$ and $\Delta$ must be small with respect to $r$, it can be shown that the displacement thickness $\delta^{*}$ has the same physical meaning as it did for a thin boundary layer (Eq. 8) when $\delta^{*}$ is related to a new integral given by

$$
\delta^{*}\left(1-\frac{\delta^{*}}{2 r}\right)=\int_{0}^{\delta_{r}^{\prime}}\left(1-\frac{\bar{\rho} \bar{u}}{\rho U}\right)\left(1-\frac{y \cos \alpha}{r}\right) d y
$$

where $\alpha$ is the angle the wall makes with respect to the centerline at the local station $z$. Making use of this thick boundary-layer definition of $\delta^{*}$, it can be shown that the deficiency of momentum of the real flow near the wall compared with the same mass flux of potential flow is

$$
\dot{M}_{p}-\dot{M}_{r}=2 \pi \rho U^{2} r \theta\left(1-\frac{\theta}{2 r}\right)=2 \pi \rho U^{2} \int_{0}^{\delta_{r}^{\prime}} \frac{\bar{\rho} \bar{u}}{\rho U}\left(1-\frac{\bar{u}}{U}\right)\left(1-\frac{y \cos \alpha}{r}\right) d y
$$

and similarly with the enthalpy flux of the real flow near the wall,

$$
\begin{array}{r}
\dot{H}_{p}-\dot{H}_{r}=2 \pi \rho U c_{p}\left(T_{0}-T_{w}\right) r \phi\left(1-\frac{\phi}{2 r}\right)=2 \pi \rho U c_{p}\left(T_{0}-T_{w}\right) r \int_{0}^{\delta_{r}^{\prime}} \frac{\bar{\rho} \vec{u}}{\rho U}\left(1-\frac{\bar{t}_{0}-T_{w}}{T_{0}-T_{w}}\right) \\
\quad \times\left(1-\frac{y \cos \alpha}{r}\right) d y
\end{array}
$$

From these equations, it can be shown that the integral momentum and energy equations are identical to those for the thin boundary layer (Eqs. 25 and 30) if $\theta, \delta^{*}$, and $\phi$ are replaced by $\theta, \delta^{*}$, and $\varphi$, where

$$
\delta^{*}=\delta^{*}\left(1-\frac{\delta^{*}}{2 r}\right) \quad \boldsymbol{\theta}=\theta\left(1-\frac{\theta}{2 r}\right) \quad \boldsymbol{\varphi}=\phi\left(1-\frac{\theta}{2 r}\right)
$$




\section{NOMENCLATURE}

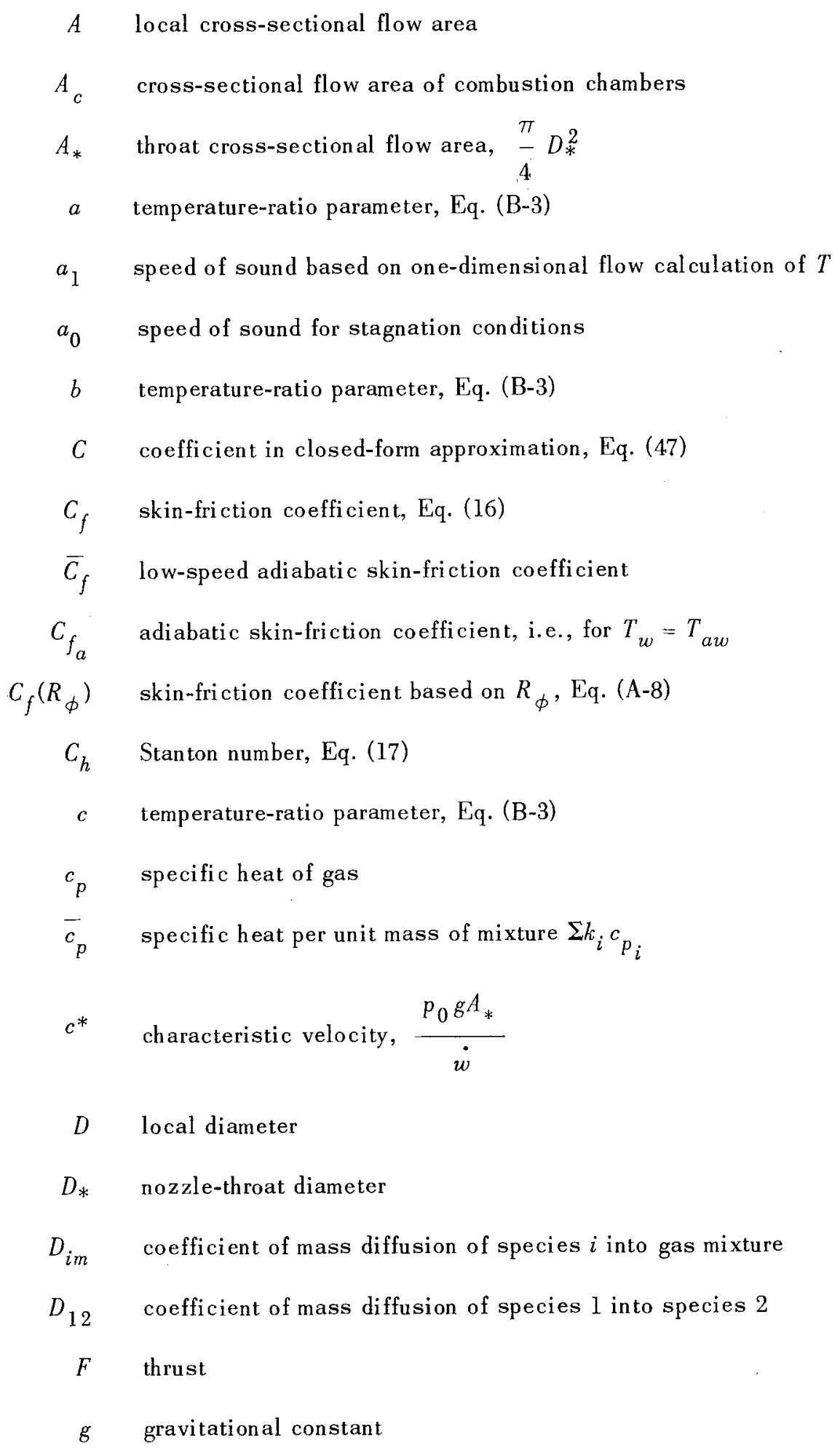




\title{
NOMENCLATURE (Cont'd)
}

\author{
$\dot{H} \quad$ en thalpy flux of wall layer \\ $h_{g} \quad$ heat-transfer coefficient based on temperature driving potential \\ $h_{i} \quad$ heat-transfer coefficient based on enthalpy driving potential, $h_{g} / c_{p}$ \\ $I \quad$ static enthalpy per unit mass at edge of boundary layer \\ $I_{f} \quad$ frozen static enthalpy per unit mass at edge of boundary layer, $\Sigma K_{i} \int_{0}^{T} c_{p_{i}} d T$ \\ $\Delta I_{\text {chem }} \quad$ chemical enthalpy per unit mass, $\Sigma\left(K_{i}-K_{i_{w}}\right) I_{i}^{(0)}$ \\ $l_{i}^{(0)} \quad$ enthalpy of formation per unit mass at standard conditions \\ $\Delta l_{\text {kinetic }} \quad$ portion of total enthalpy per unit mass represented by kinetic energy, $1 / 2 U^{2}$ \\ $I_{0} \quad$ Total or stagnation enthalpy per unit mass at edge of boundary layer \\ $I_{w} \quad$ enthalpy per unit mass of gas mixture at $T_{w}$ \\ $I_{1} \cdots I_{7} \quad$ definite integrals in boundary-layer shape parameter expressions, \\ Eqs. (B-6) through (B-9) \\ $i \quad$ complete local static enthalpy per unit mass of mixture of gases within \\ boundary layer, $\Sigma k_{i} i_{i}$ \\ $i_{i} \quad$ local static enthalpy per unit mass of $i$ th species within boundary layer \\ $K_{i} \quad$ mass fraction of $i$ th species at edge of boundary layer \\ $K_{i_{w}} \quad$ mass fraction of $i$ th species at wall \\ $k \quad$ mass-flow rate exponent, Eq. (46) \\ $k_{i} \quad$ local mass fraction of $i$ th species within boundary layer \\ $L e_{f} \quad$ Lewis - Semenov number $\rho D_{12} \bar{c}_{p} / \lambda$ \\ $L^{*} \quad$ characteristic length (combustion volume $/ A_{*}$ ) \\ $L \quad$ nozzle length along axis \\ 1 combustion chamber or approach duct length \\ $M \quad$ Mach number at edge of boundary layer \\ $\dot{M} \quad$ momentum flux of wall layer, Eqs. (2), (5) \\ M molecular weight of gas mixture \\ $m \quad$ exponent of temperature dependence of viscosity
}




\section{NOMENCLATURE (Cont'd)}

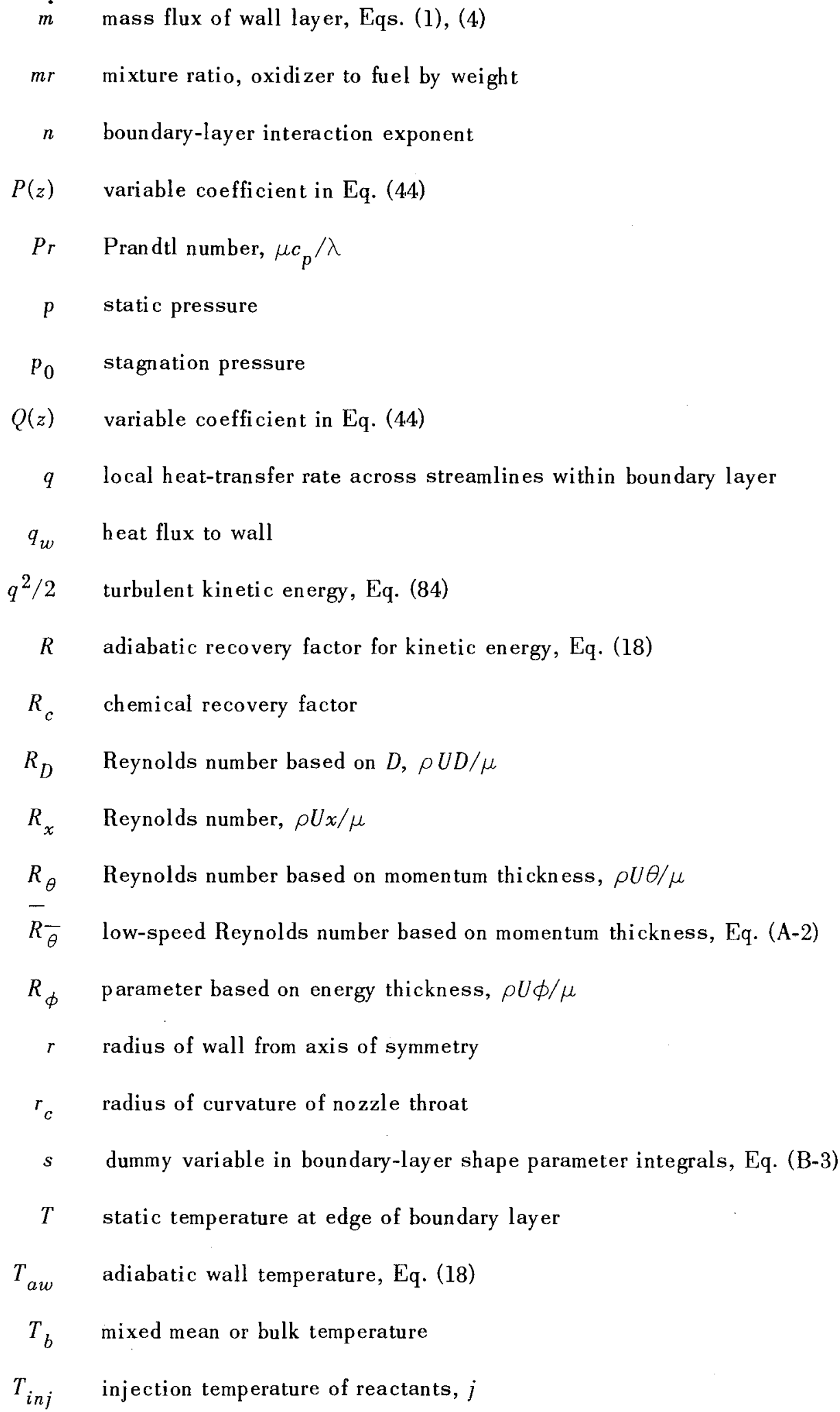




\title{
NOMENCLATURE (Cont'd)
}

\author{
$T_{m} \quad$ log-mean bulk temperature (46) \\ $T_{0} \quad$ stagnation temperature \\ $T_{\text {ref }}$ thermodynamic reference temperature, $298^{\circ} \mathrm{K}$ \\ $T_{s} \quad$ sublayer temperature in Coles' transformation, Eq. (A-3) \\ $T_{w} \quad$ wall temperature \\ $t \quad$ local static temperature within boundary layer \\ $\bar{t}_{0} \quad$ local time-mean stagnation temperature in boundary layer \\ $U \quad$ velocity at edge of velocity boundary layer \\ $\bar{u} \quad$ local time-mean $x$-component of velocity in boundary layer \\ $u^{+} \quad$ dimensionless velocity parameter, $\bar{u} / \sqrt{\tau_{w} / \rho}$ \\ $u, v \quad$ components of velocity parallel and normal to surface, respectively \\ $v_{i} \quad$ local scalar diffusion velocity of $i$ th species within boundary layer \\ $w \quad$ dummy variable in boundary-layer shape parameter integrals, Eq. (B-7) \\ $\dot{w} \quad$ weight rate of flow per unit time through nozzle \\ $x \quad$ distance along wall \\ $y \quad$ distance from wall along normal \\ $y^{+}$dimensionless distance from wall, $\left(y \sqrt{\left.\tau_{w} / \rho\right) / \nu}\right.$ \\ $z \quad$ distance along axis of symmetry \\ a angle between wall and axis at station $z$ \\ $\gamma$ ratio of specific heat at constant pressure to that at constant volume \\ $\Delta \quad$ thickness of temperature boundary layer \\ $\delta \quad$ thickness of velocity boundary layer \\ $\delta^{\prime} \quad$ thickness of layer containing all wall effects \\ $\delta^{*} \quad$ displacement thickness of boundary layer, Eqs. (8), (9) \\ $\delta^{*} \quad$ thick boundary-layer displacement thickness parameter $\delta^{*}\left(1-\delta^{*} / 2 r\right)$
}




\title{
NOMENCLATURE (Cont'd)
}

\author{
$\epsilon_{c} \quad$ contraction ratio $A_{c} / A_{*}$ \\ $\zeta \quad$ boundary-layer thickness ratio $(\Delta / \delta)^{1 / 7}$ \\ $\theta$ momentum thickness of boundary layer, Eqs. (11), (12) \\ $\theta$ thick boundary-layer momentum-thickness parameter, Eq. (C-4) \\ $\lambda \quad$ thermal conductivity \\ $\mu \quad$ viscosity at edge of boundary lay er \\ $\mu_{0} \quad$ viscosity of gas at stagnation conditions \\ $\mu_{s} \quad$ viscosity of gas evaluated at $T_{s}$ \\ $\nu \quad$ kinematic viscosity \\ $\rho \quad$ gas density at edge of boundary layer \\ $\bar{\rho}$ local time-mean density in boundary layer \\ $\sigma \quad$ variable properties correction factor, Eqs. (49), (58) \\ $\sigma^{\prime} \quad$ general variable properties correction factor, Eq. (55) \\ $\mathcal{\tau}_{w} \quad$ retarding wall shear stress \\ $\phi \quad$ energy thickness of boundary layer, Eqs. (14), (15) \\ P thick boundary-layer energy thickness parameter, Eq. (C-4) \\ $\chi \quad$ turbulence decay parameter, Eq. (86)
}

Subscripts
am arithmetic mean
$a w \quad$ adiabatic wall
$b \quad$ property evaluated at mixed mean or bulk temperature $T_{b}$
$f \quad$ "frozen," evaluated based on absence of chemical change
$i \quad i$ th species (products)
$j \quad$ jth species (reactants) 


\section{NOMENCLATURE (Cont'd)}

$\begin{array}{ll}o & \text { entrance or initial condition } \\ p & \text { potential flow } \\ R & \text { recovery condition } \\ r & \text { real flow } \\ \text { ref } & \text { property evaluated at } T_{\text {ref }}=T^{*}, T_{a m}, T_{a w} \\ \text { ref } & \text { thermodynamic reference state } \\ w & \text { wall } \\ 0 & \text { stagnation } \\ 1 & \text { one-dimensional flow value } \\ \text { ( ) } & \text { fluctuating quantity }\end{array}$




\section{FIGURES}

1. Nomenclature for real flow

2. Nomenclature for adiabatic potential flow

3. Adiabatic skin-friction coefficient for low-speed flow

4. Comparison of modified Stanton number correlation (Eq. A-8) with data from low-speed flow

5. Nozzle contour and flow conditions for sample calculation

6. Velocity boundary-layer thicknesses for nozzle and conditions of Fig. 5

7. Temperature boundary-layer thicknesses for nozzle and conditions of Fig. 5

8. Displacement thickness for nozzle and conditions of Fig. 5

9. Heat-transfer coefficient for nozzle and conditions of Fig. 5

10. Heat-transfer coefficient for nozzle and conditions of Fig. 5; closed-form approximation comparison

11. Flow and instrumentation diagram; heated-air investigation of Reference 59

12. Tip details of traversing boundary-layer probes; heated-air investigation of Reference 59

13. Thermocouple plug diagram; heated-air investigation of Reference 59

14. Nozzle wall isotherms; heated-air investigation of Reference 59

15. Ratio of static to stagnation pressure along the nozzle (of Reference 59)

16. Ratio of local to one-dimensional mass flux along the nozzle (of Reference 59)

17. Boundary-layer profiles 1.25 inch upstream of nozzle inlet with 18 -inch cooled approach 1 ength (59)

18. Heat-transfer coefficient vs axial distance ratio with 18-inch cooled approach length (59)

19. Comparison of measured and predicted heat-transfer coefficients for heated air at $p_{0}=75 \mathrm{psia}(59$, 14)

20. Comparison of measured and predicted heat-transfer coefficients for heated air at $p_{0}=254 \mathrm{psia}$ $(59,14)$

21. Comparison of measured and predicted heat-transfer coefficients for various boundary-layer thicknesses at nozzle inlet for heated air (59)

22. Comparison of measured and predicted heat-transfer coefficients for heated air at $p_{0}=44.8 \mathrm{psia}(59)$

23. Correlation of $C_{h} \operatorname{Pr}^{0.6}$ versus $R_{D}$ at various subsonic and supersonic area ratios, with 18-inch cooled approach length (59)

24. Predicted momentum-thickness Reynolds numbers along nozzle (59)

25. Comparison of measured and predicted heat-transfer coefficients for air (data from Reference 57) 


\section{FIGURES (Cont'd)}

26. Comparison of measured and predicted heat-transfer coefficients for air (data from Reference 58)

27. Predicted effect of flow acceleration in reducing net production of turbulent kinetic energy at different stagnation pressures (59)

28. Sectional, water-cooled thrust-chamber assembly for steady-state calorimetric measurements of semilocal heat flux $(68)$

29. Surface thermocouple plug assembly for transient measurement of local heat flux (72)

30. Propellant injectors (52)

31. Comparison of predicted values with experimental heat-flux measurements made with 4-to-1 contraction-area-ratio nozzle at low chamber pressure (52)

32. Comparison of predicted values with experimental heat-flux measurements made with 8 -to-1 contraction-area-ratio nozzle at low chamber pressure (52)

33. Comparison of predicted values with experimental heat-flux measurements made with 1.64-to-1 contraction-area-ratio nozzle at low chamber pressure (52)

34. Comparison of experimental heat-flux measurements obtained using enzian and showerhead injectors $(52,73)$

35. Distributions of experimentally measured heat flux in combustion chambers operating at thrust levels from 1,000 to $20,000 \mathrm{lb}$ with several liquid propellants.

36. Dimensionless parameter correlation of heat-transfer coefficients in 8-to-1 contraction-area-ratio nozzle operating with $\mathrm{N}_{2} \mathrm{O}_{4}-\mathrm{N}_{2} \mathrm{H}_{4}$ propellants (68)

37. Dimensionless parameter correlation of heat-transfer coefficients in 4-to-1 contraction-area-ratio nozzle operating with $\mathrm{N}_{2} \mathrm{O}_{4}-\mathrm{N}_{2} \mathrm{H}_{4}$ propellants (68)

38. Dimensionless parameter correlation of heat-transfer coefficients in 2.5-to-1 contraction-area-ratio nozzle operating with $\mathrm{N}_{2} \mathrm{O}_{4}-\mathrm{N}_{2} \mathrm{H}_{4}$ propellants (68)

39. Dimensionless parameter correlation of heat-transfer coefficients in 1.64-to- 1 contraction-area-ratio nozzle operating with $\mathrm{N}_{2} \mathrm{O}_{4}-\mathrm{N}_{2} \mathrm{H}_{4}$ propellants (68)

40. Comparison of measured and predicted heat fluxes for $\mathrm{N}_{2} \mathrm{O}_{4}-\mathrm{N}_{2} \mathrm{H}_{4}$ at $p_{0}=301(68)$ 


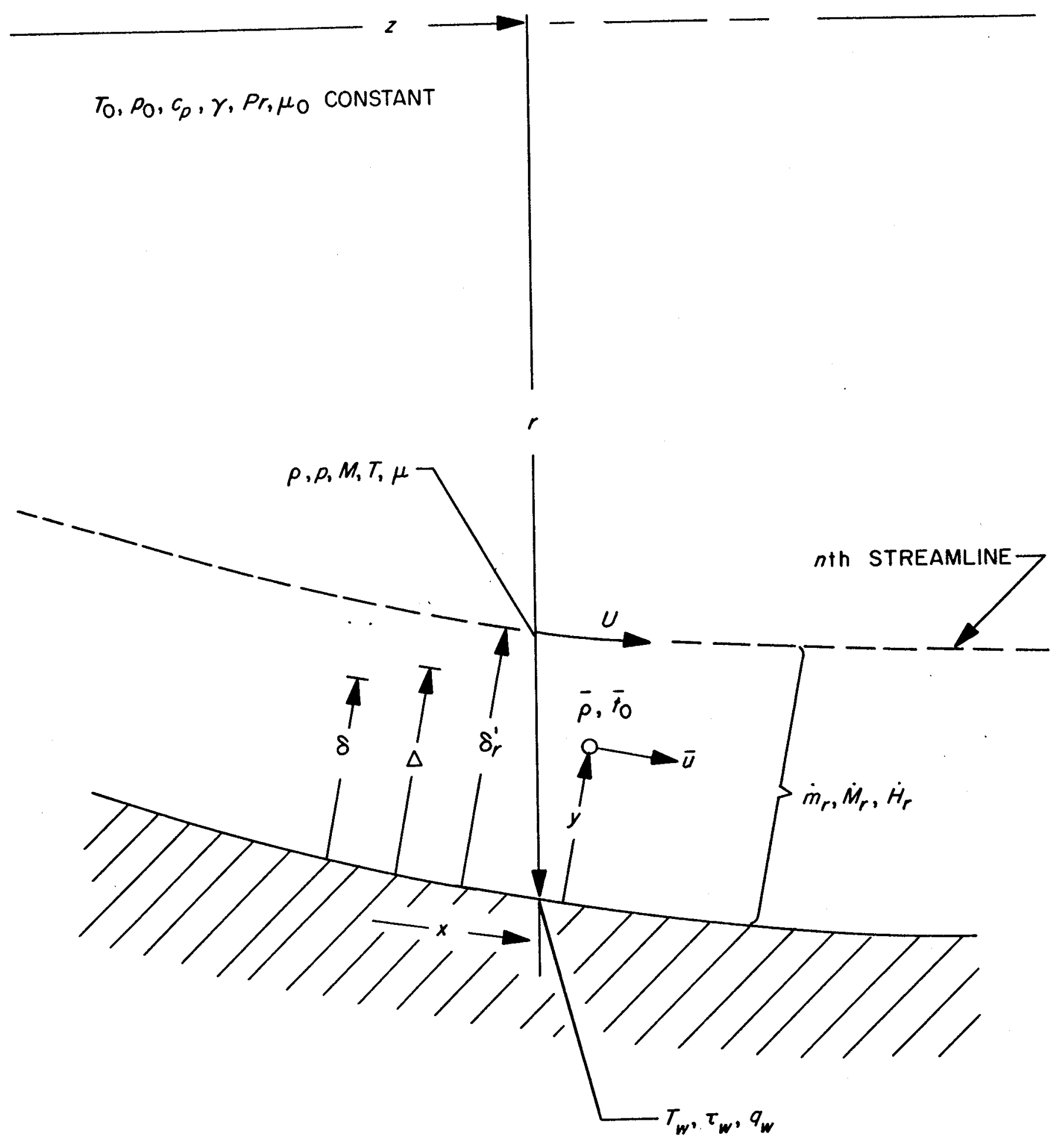



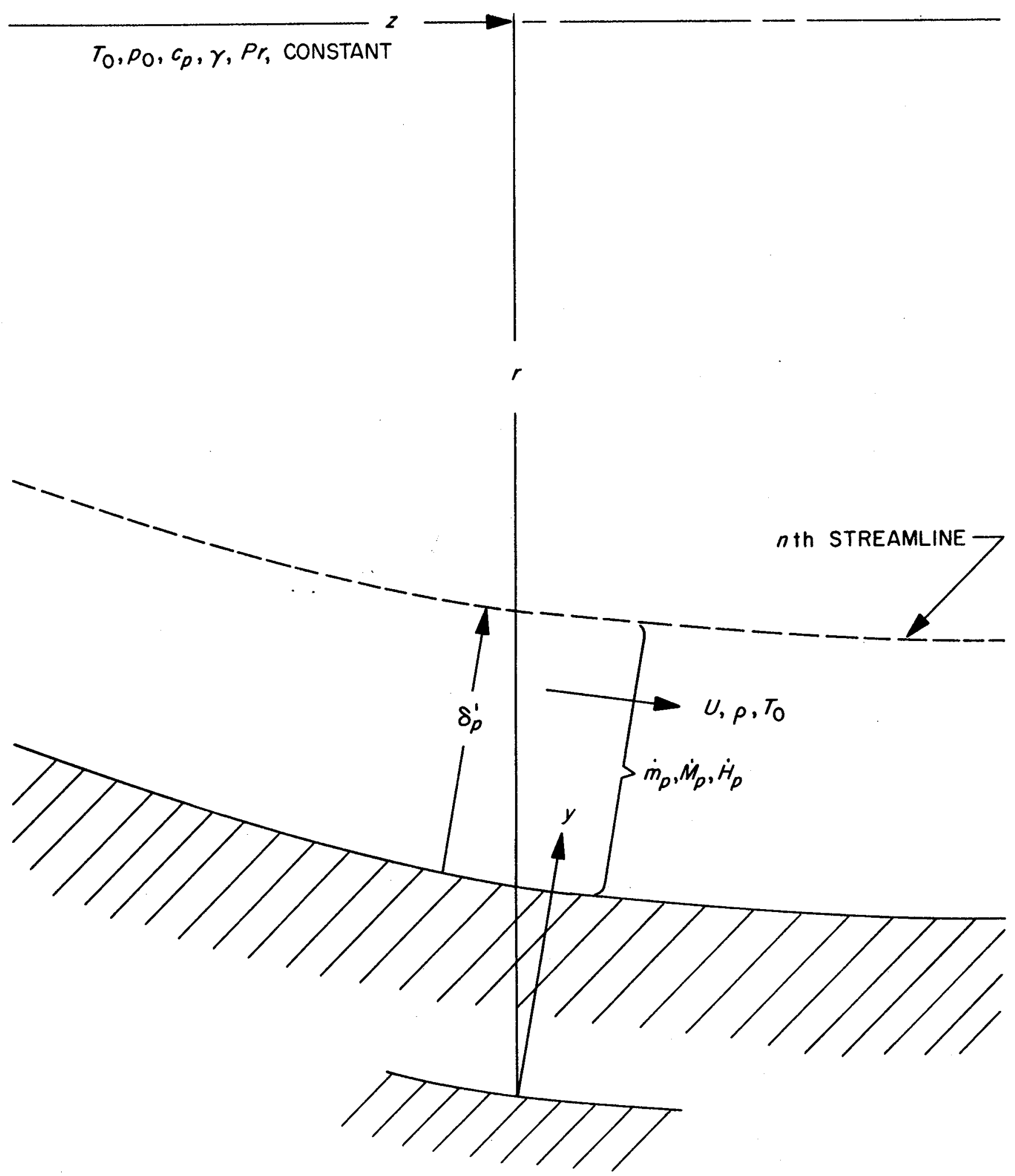


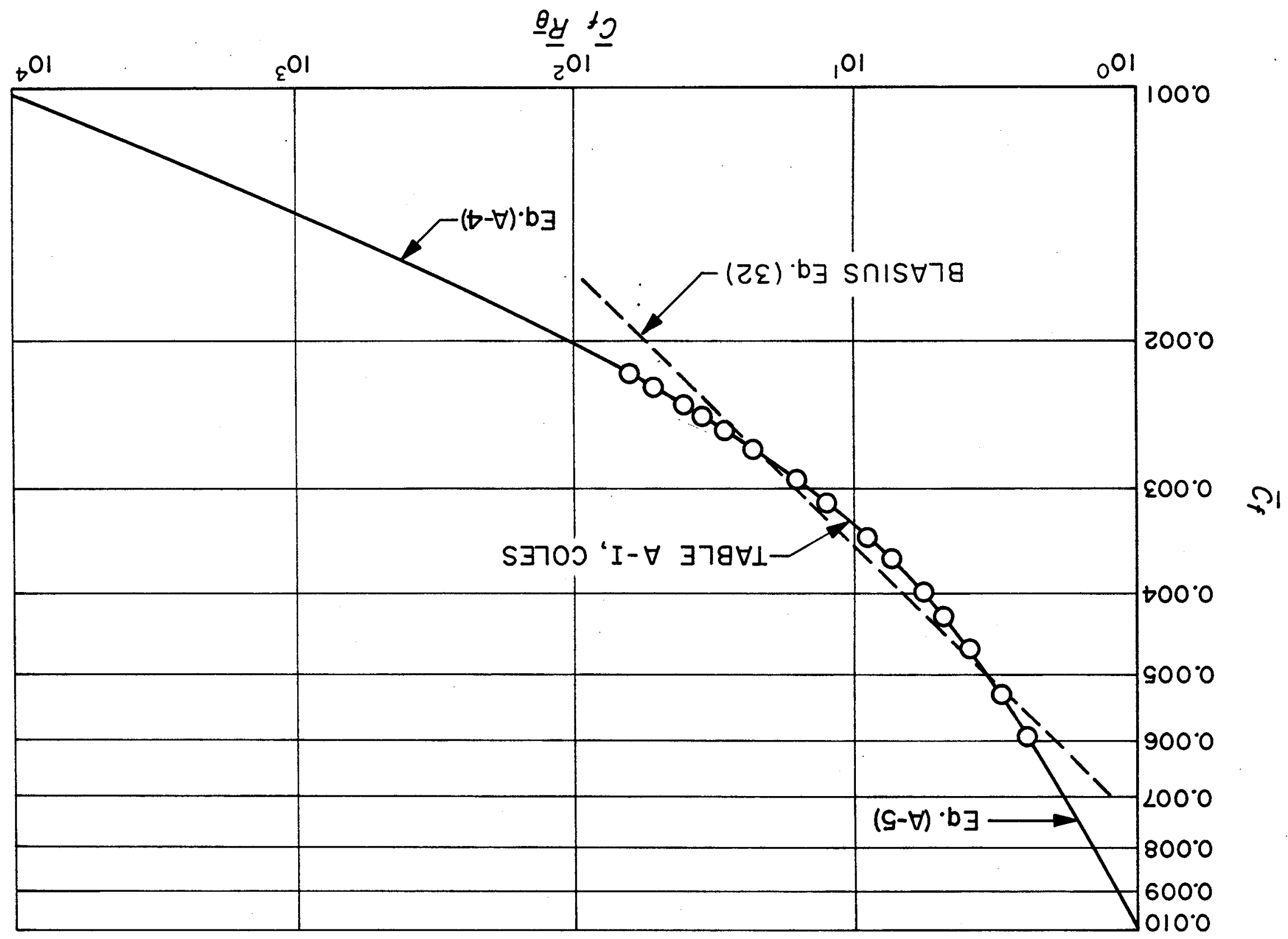




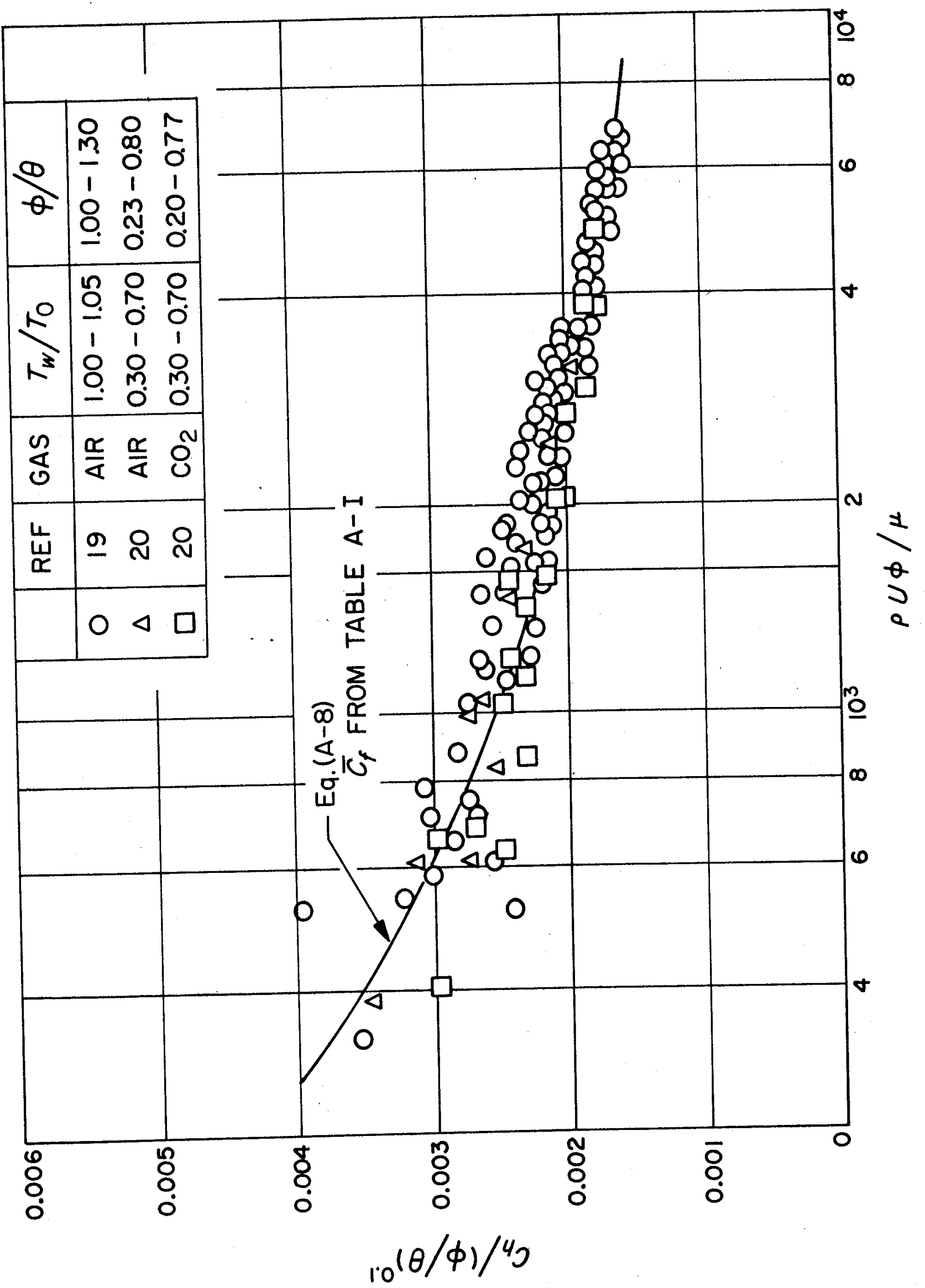


FLOW CONDITIONS

$$
\begin{aligned}
p_{0} & =300 \text { psia } \\
T_{0} & =4500{ }^{\circ} \mathrm{R} \\
c_{p} & =0.567 \text { Btu } / \mathrm{lb}{ }^{\circ} \mathrm{R} \\
P_{r} & =0.83 \\
\mu_{0} & =1.3 \times 10^{-6} \mathrm{lb} \mathrm{sec} / \mathrm{ft}^{2} \\
\gamma & =1.2 \\
\mu & \sim T^{0.65}
\end{aligned}
$$

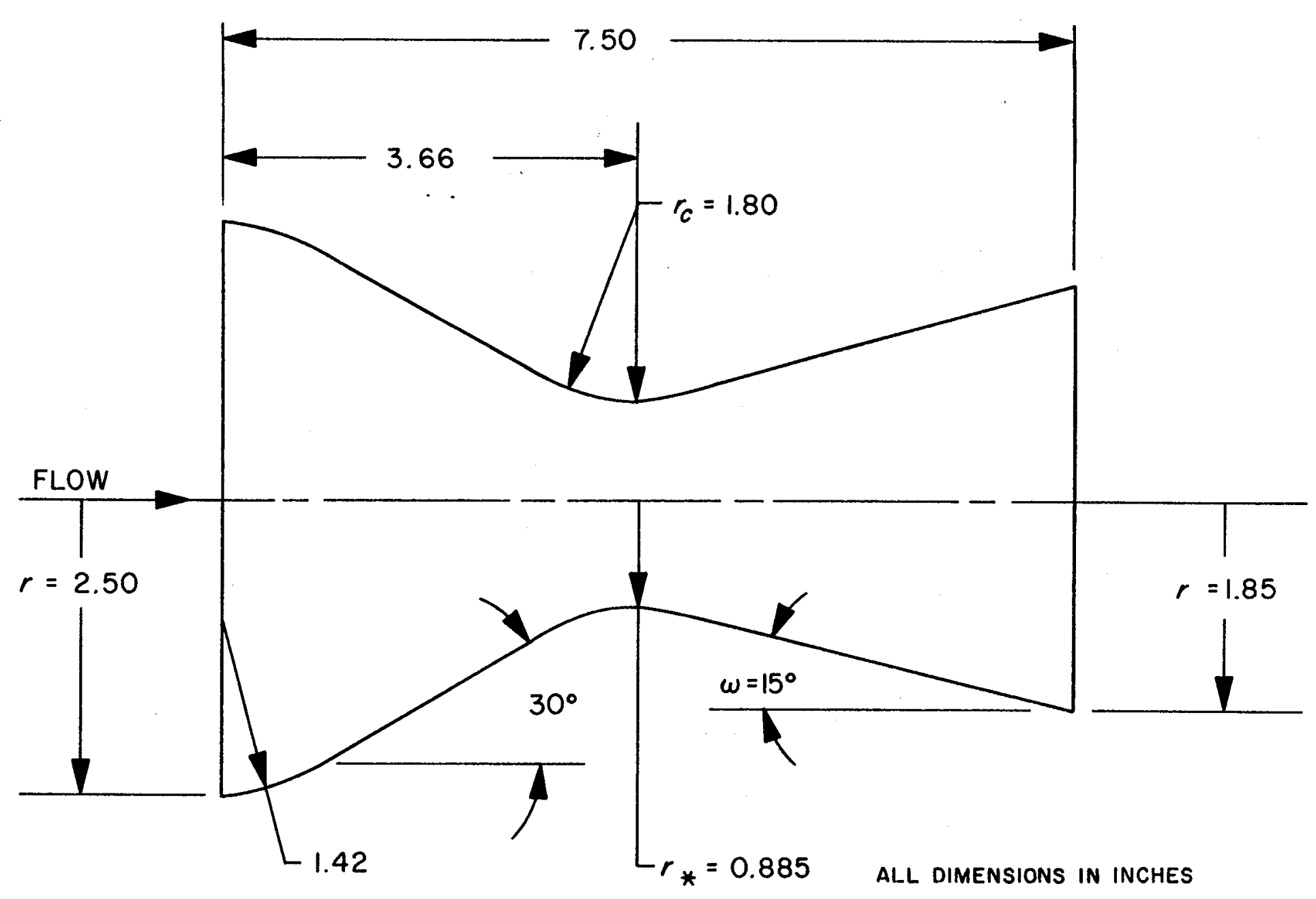




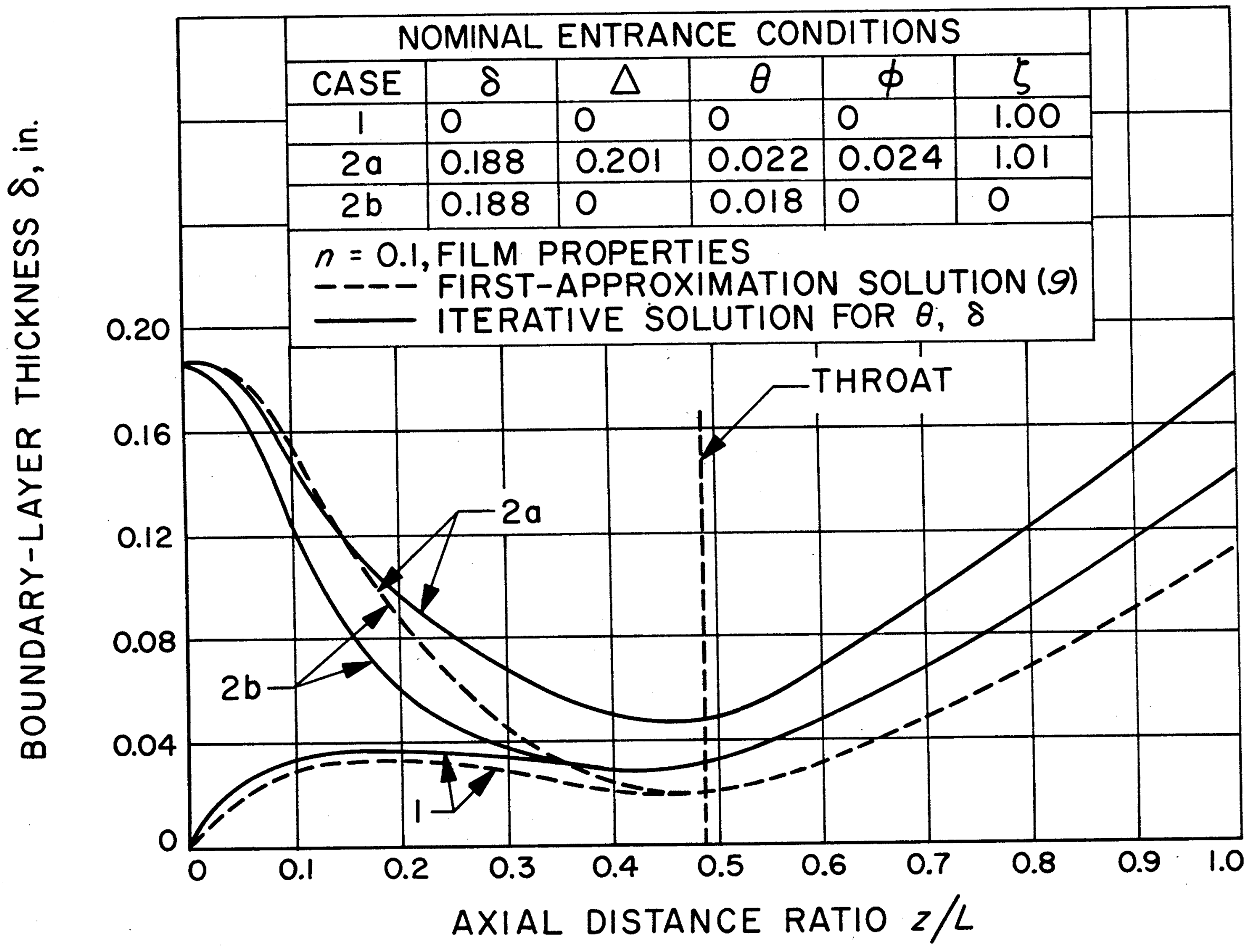




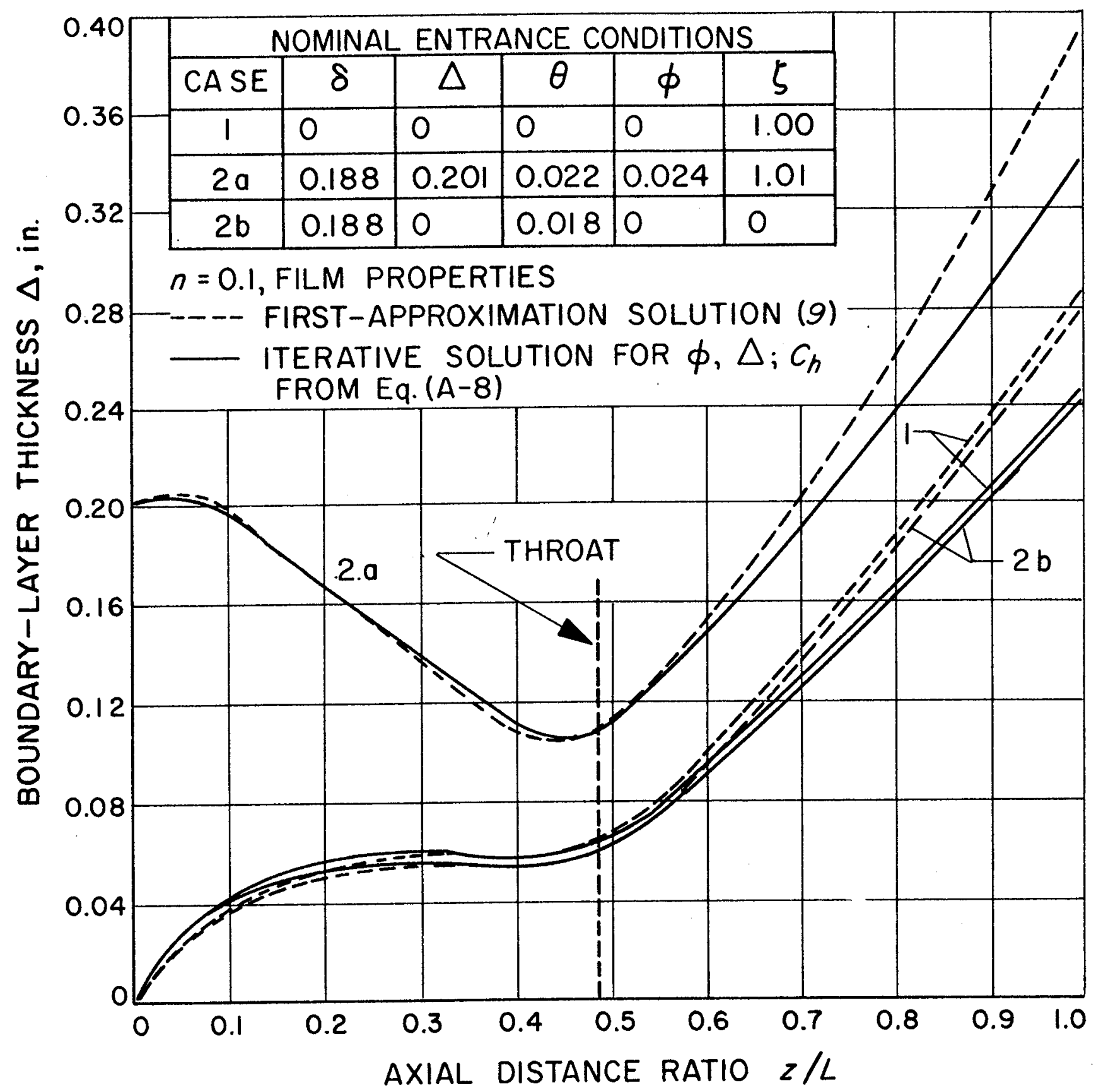




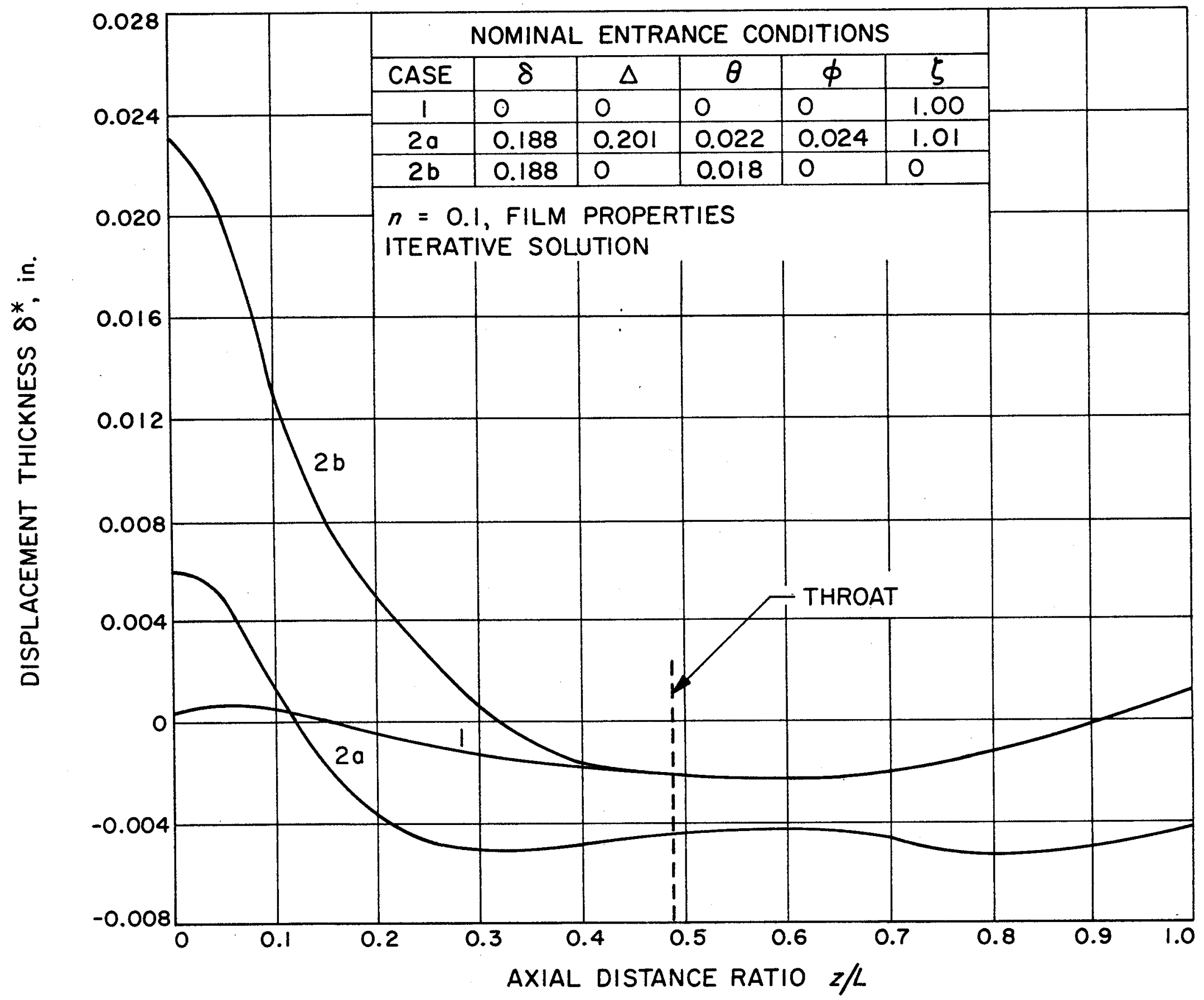




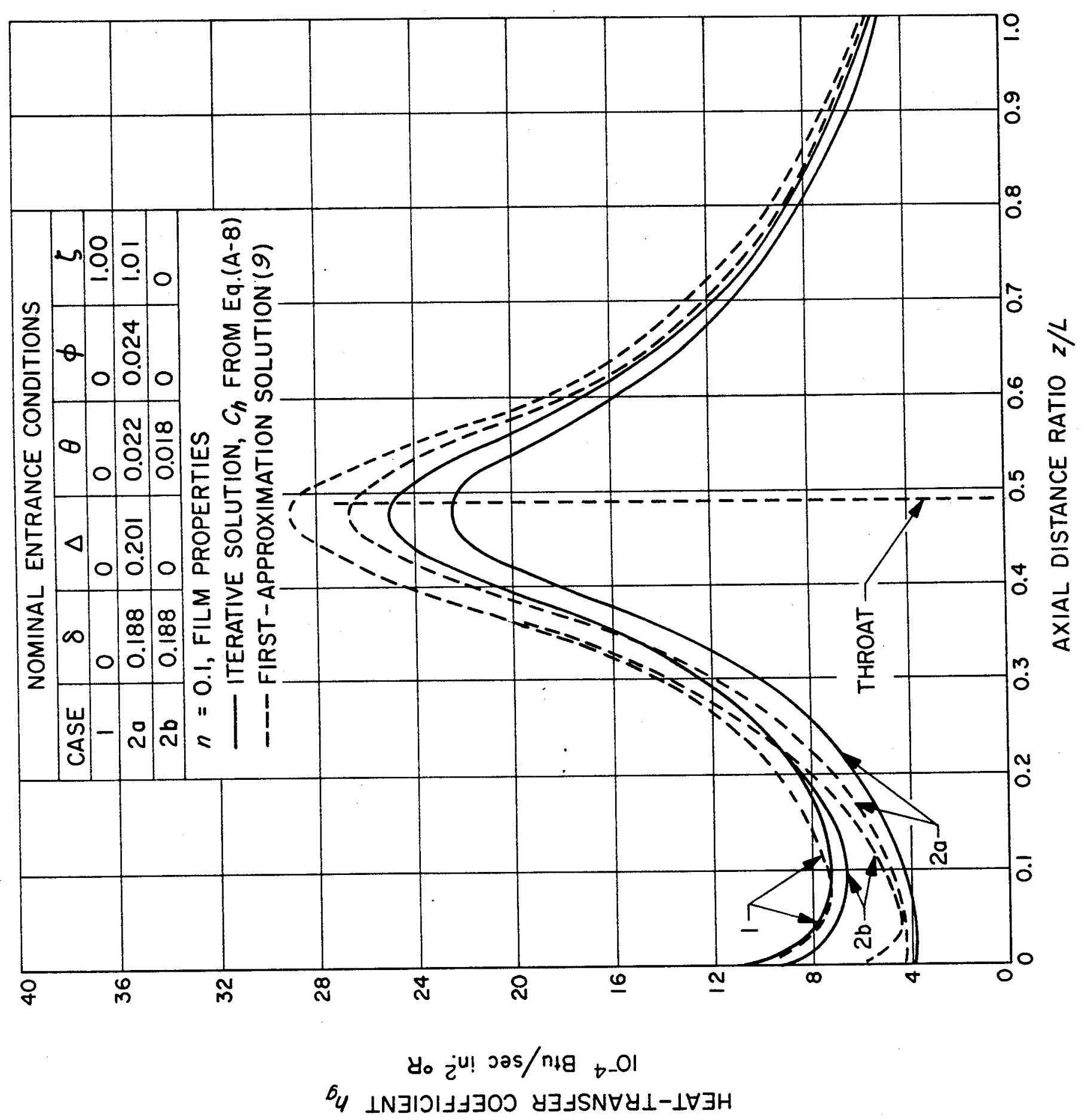




\section{HEAT-TRANSFER COEFFICIENT $h_{g}$ $10^{-4} \mathrm{Btu} / \mathrm{sec}$ in? ${ }^{\circ} \mathrm{R}$}

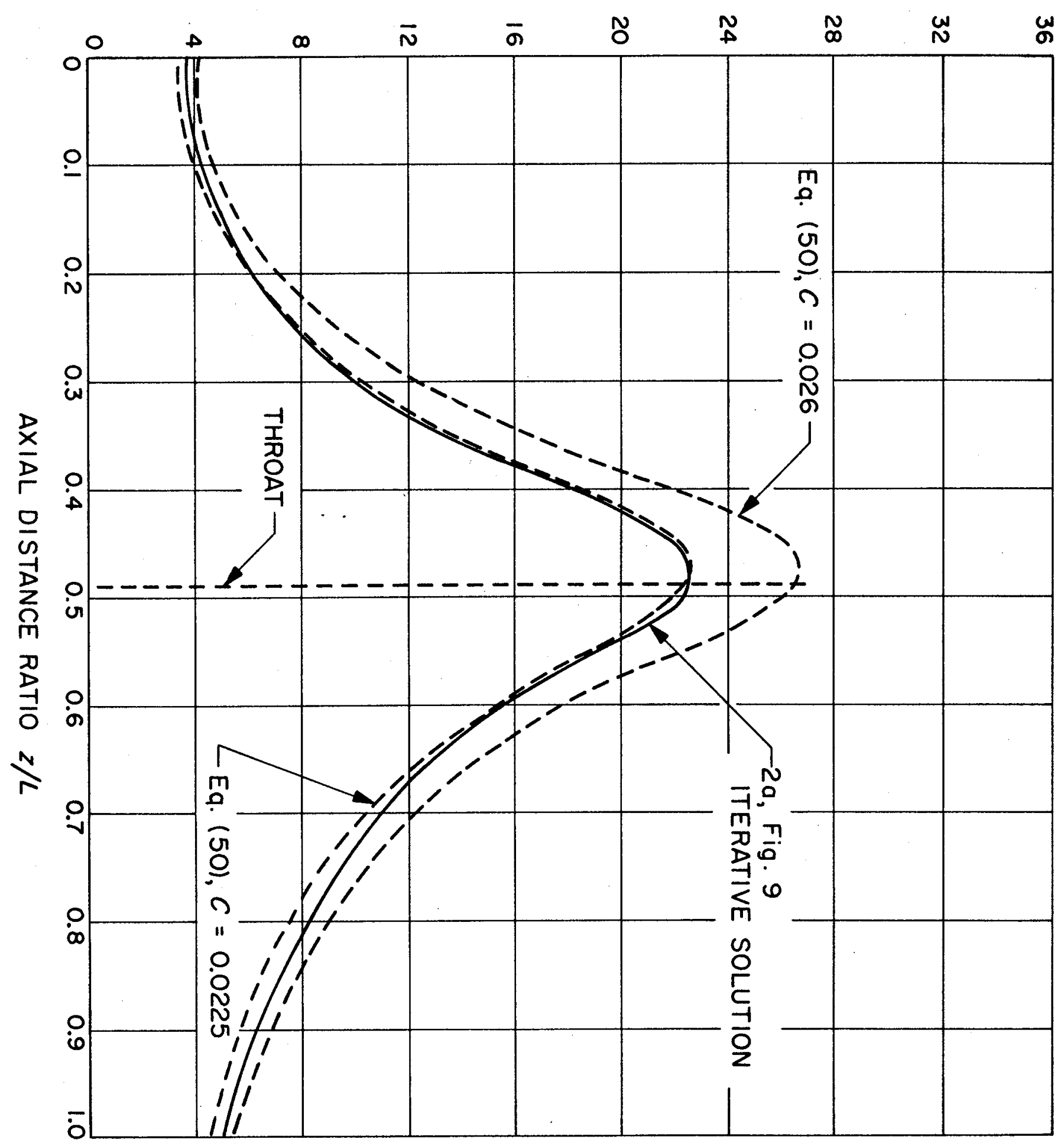




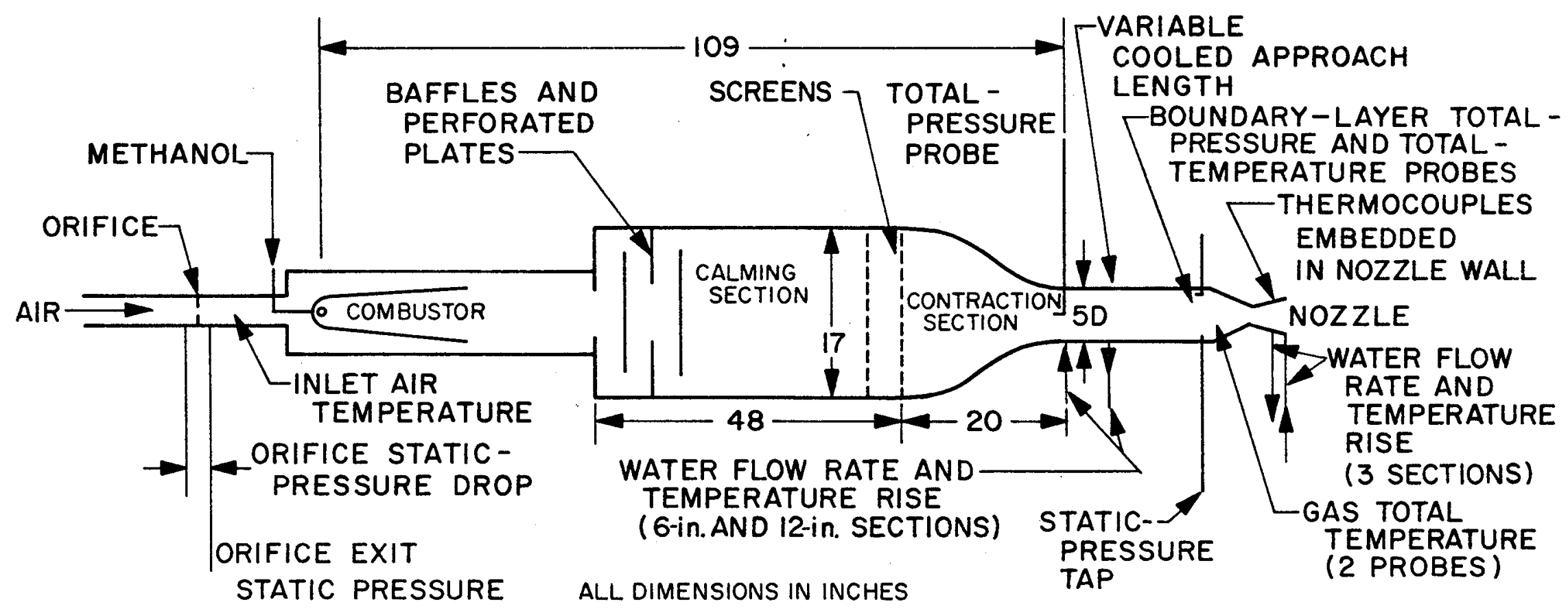


$\exists g O y d \exists y \cap \perp \forall y \exists d W \exists \perp$ NOI $\perp \forall N O \forall \perp S$ (q)

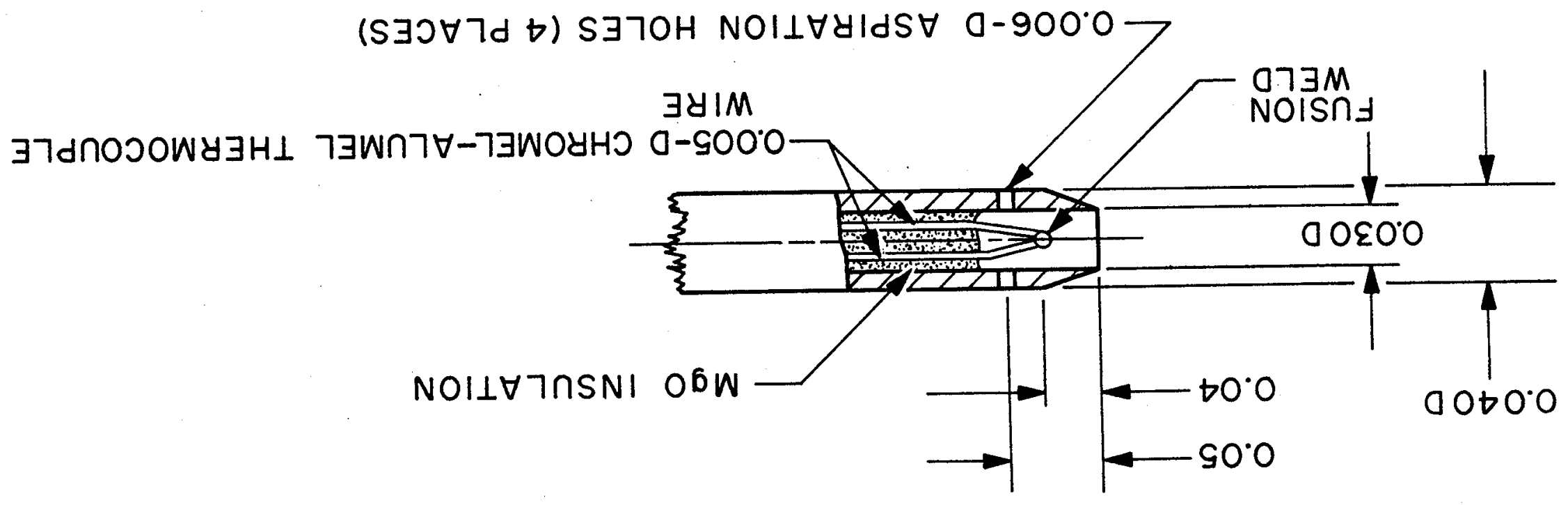

$\exists g 0 y d \quad \exists y \cap S S \exists y d-N O I \perp \forall N O \forall \perp S(D)$

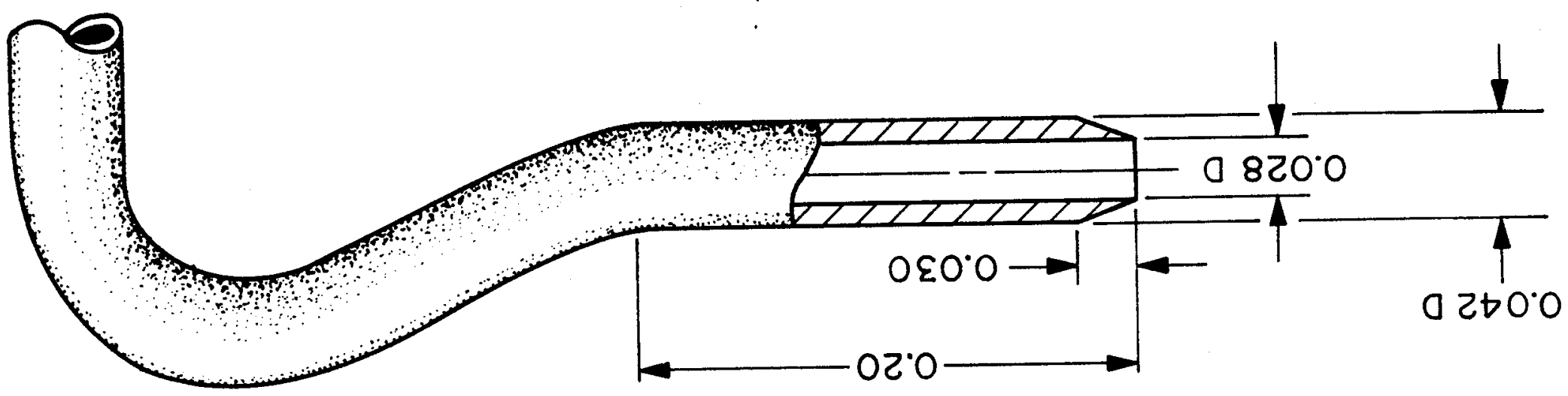




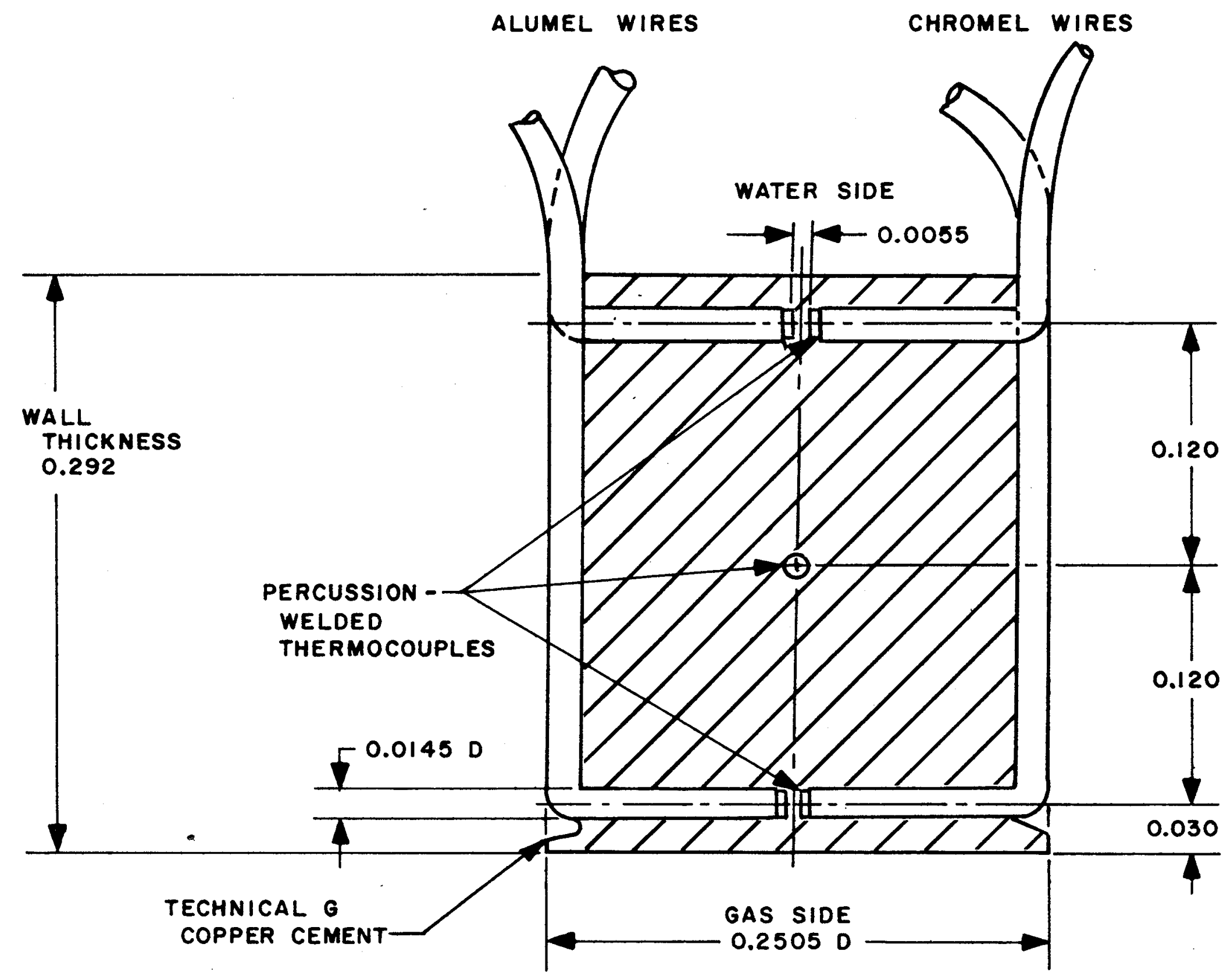

ALL DIMENSIONS IN INCHES 


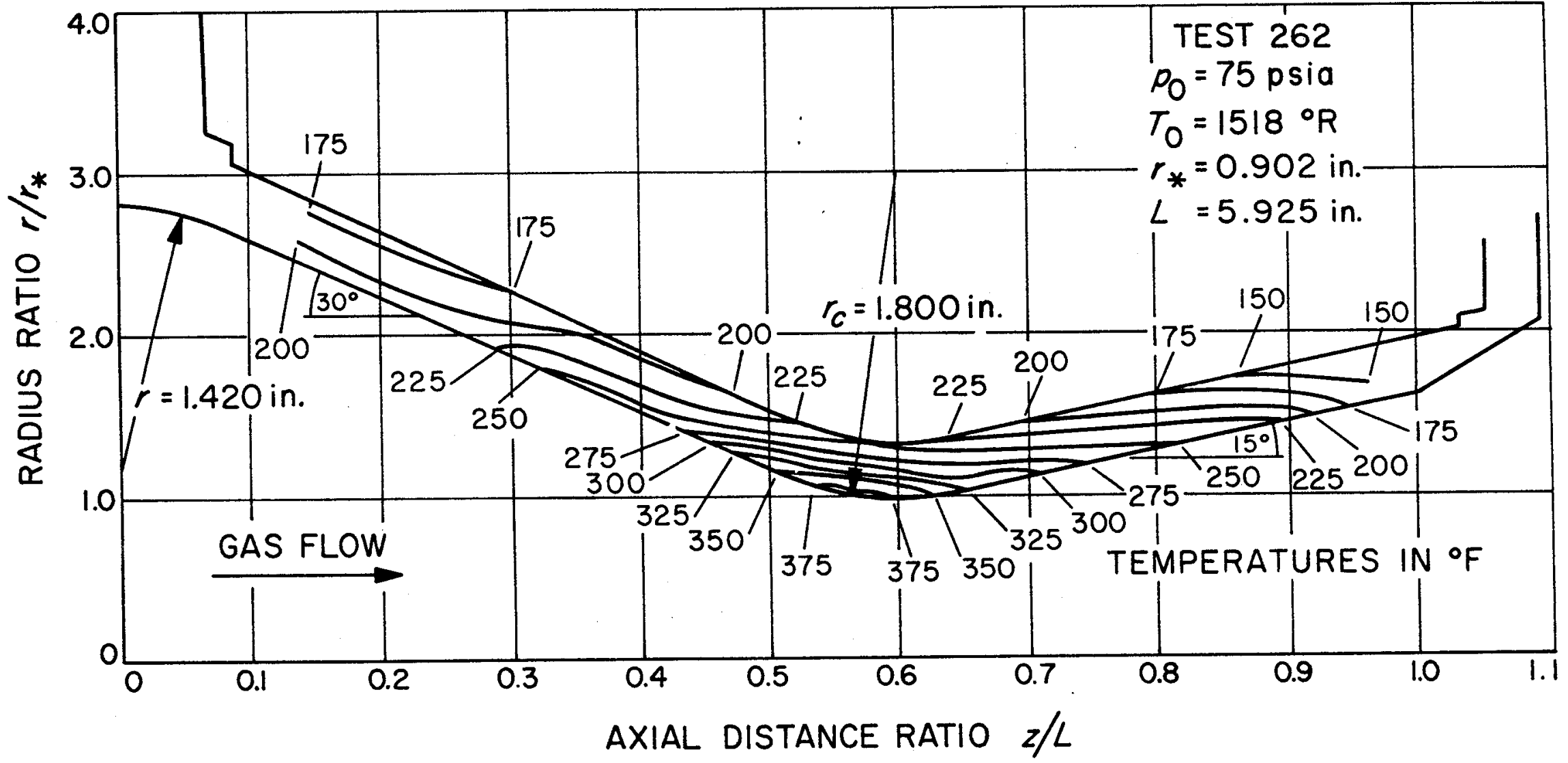




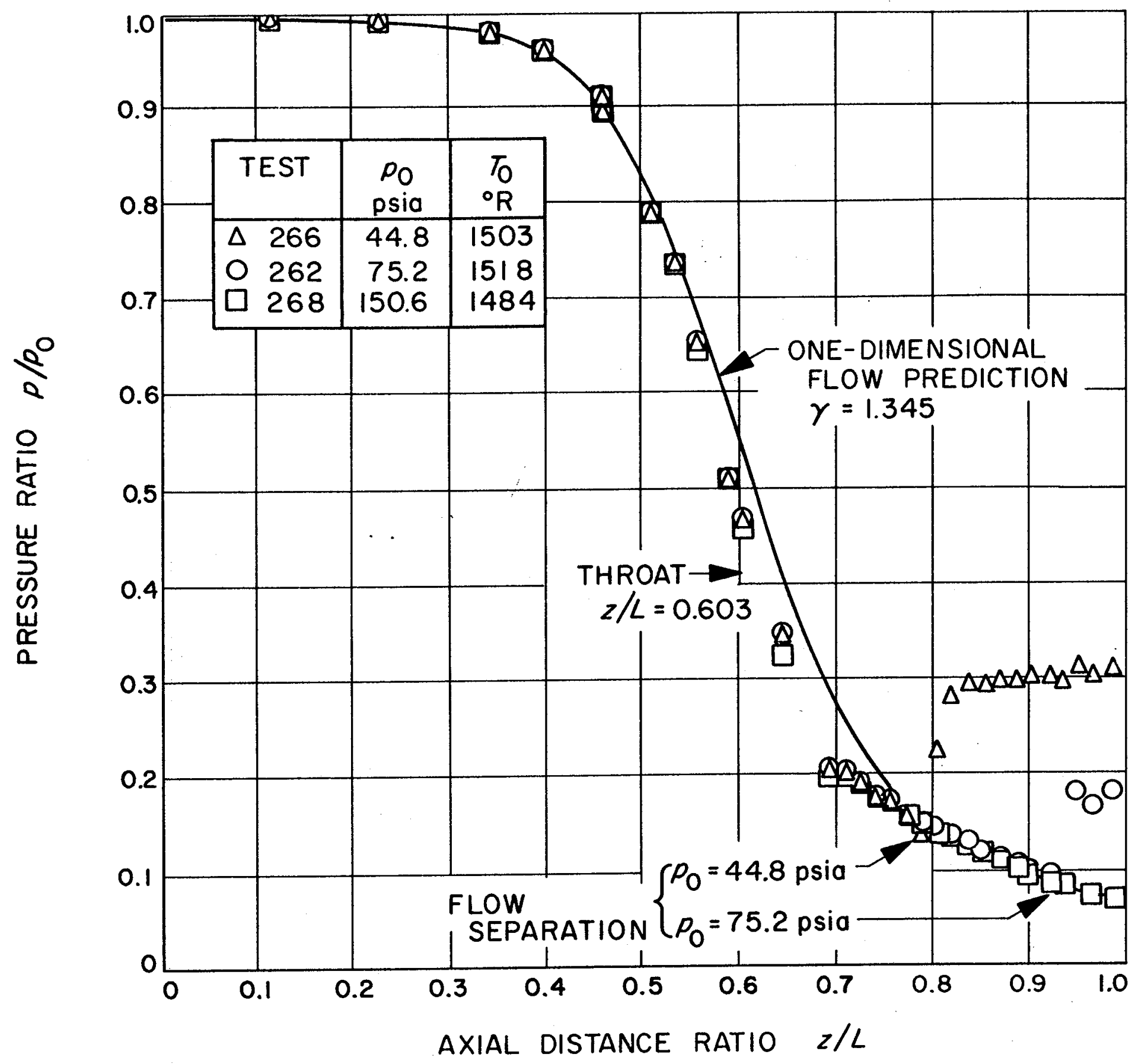




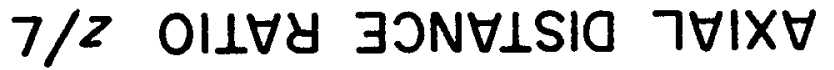

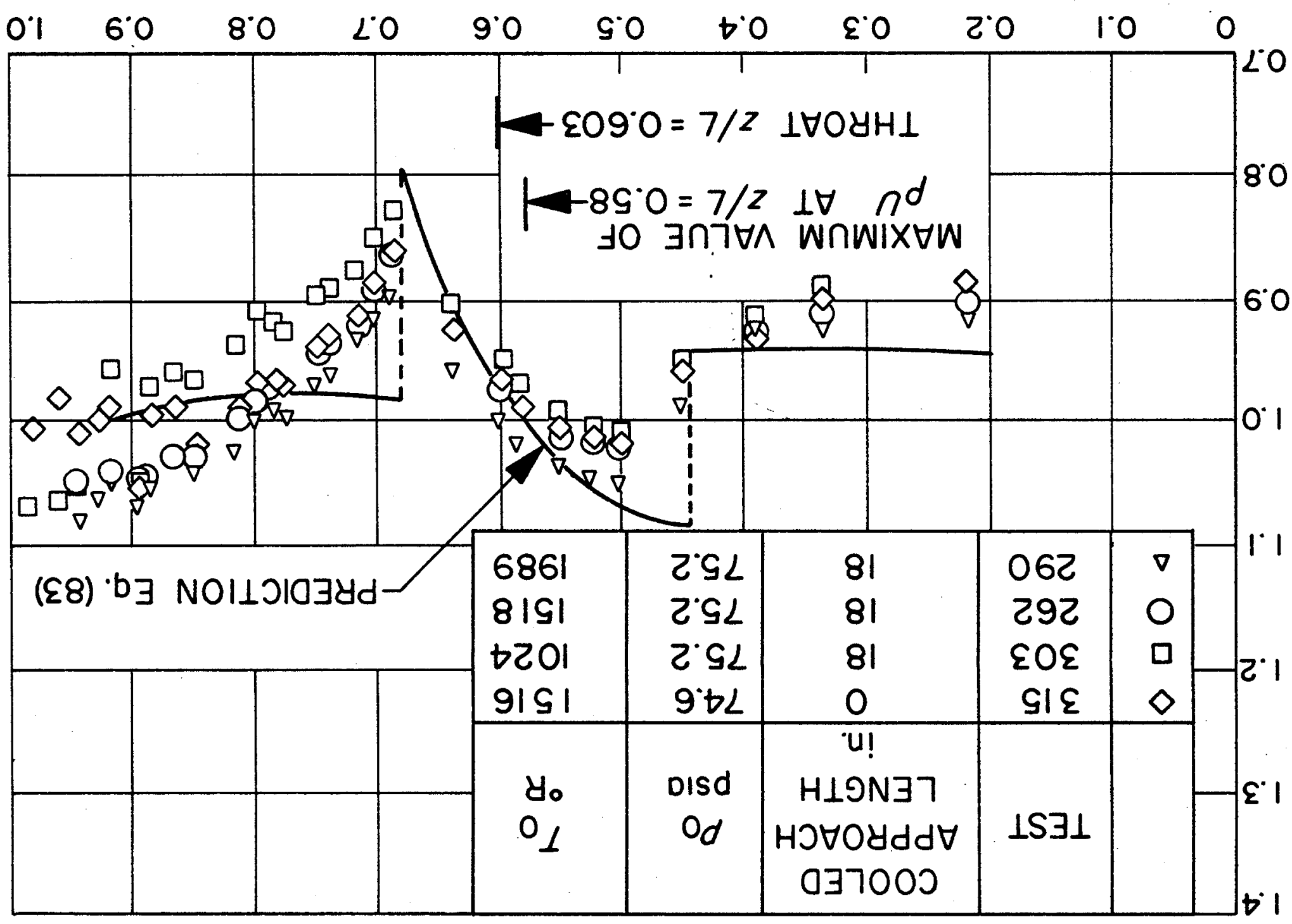



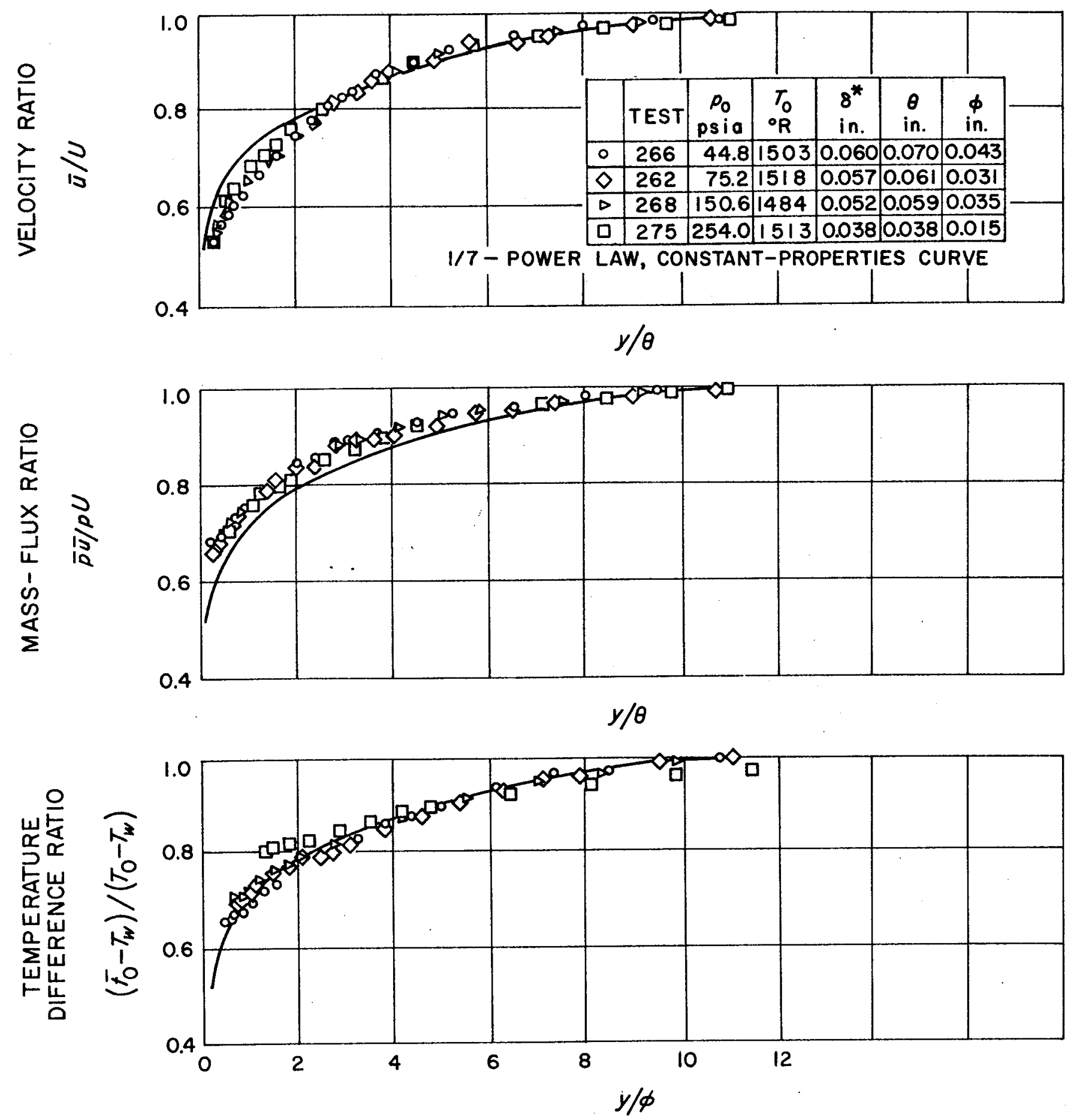


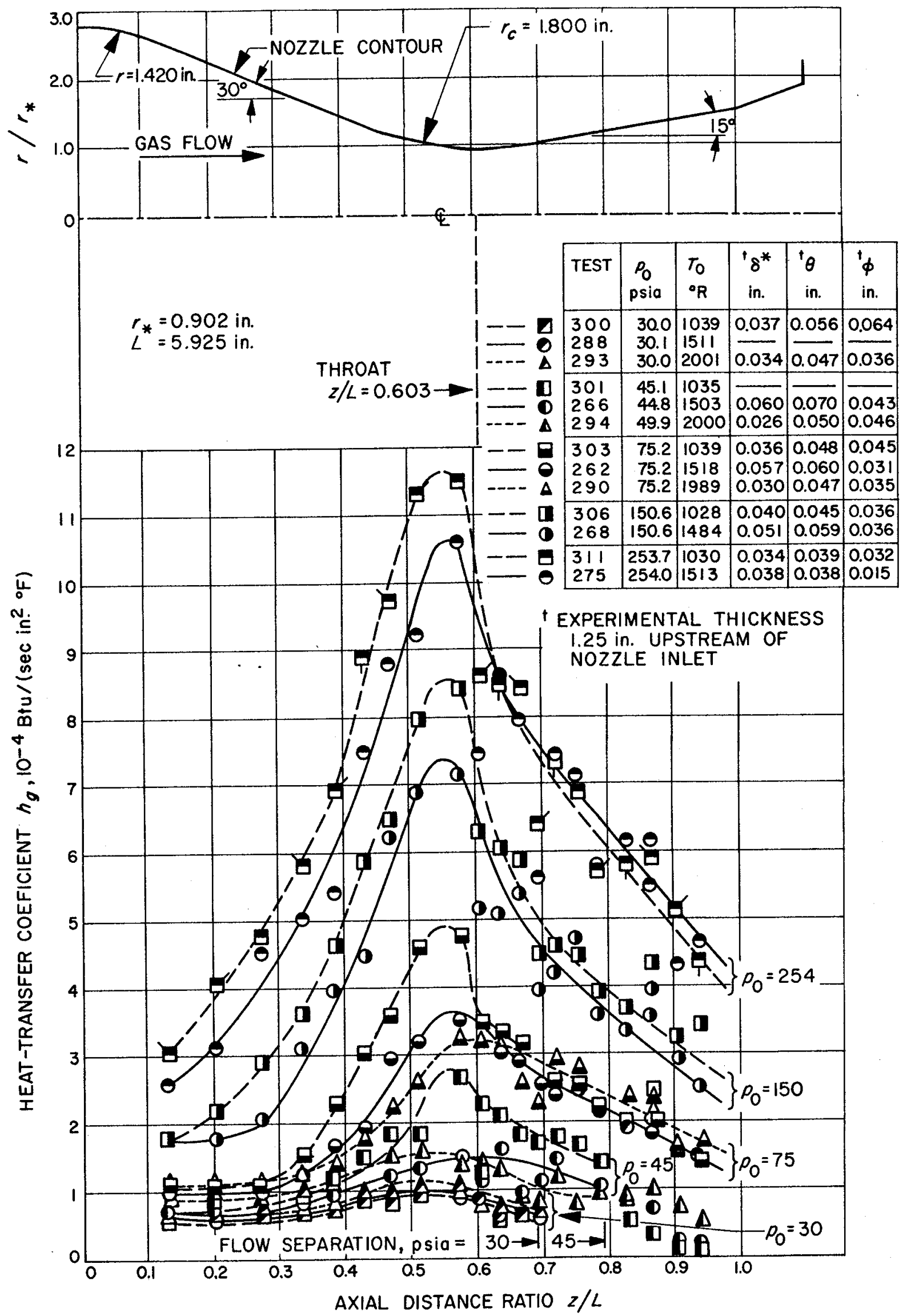




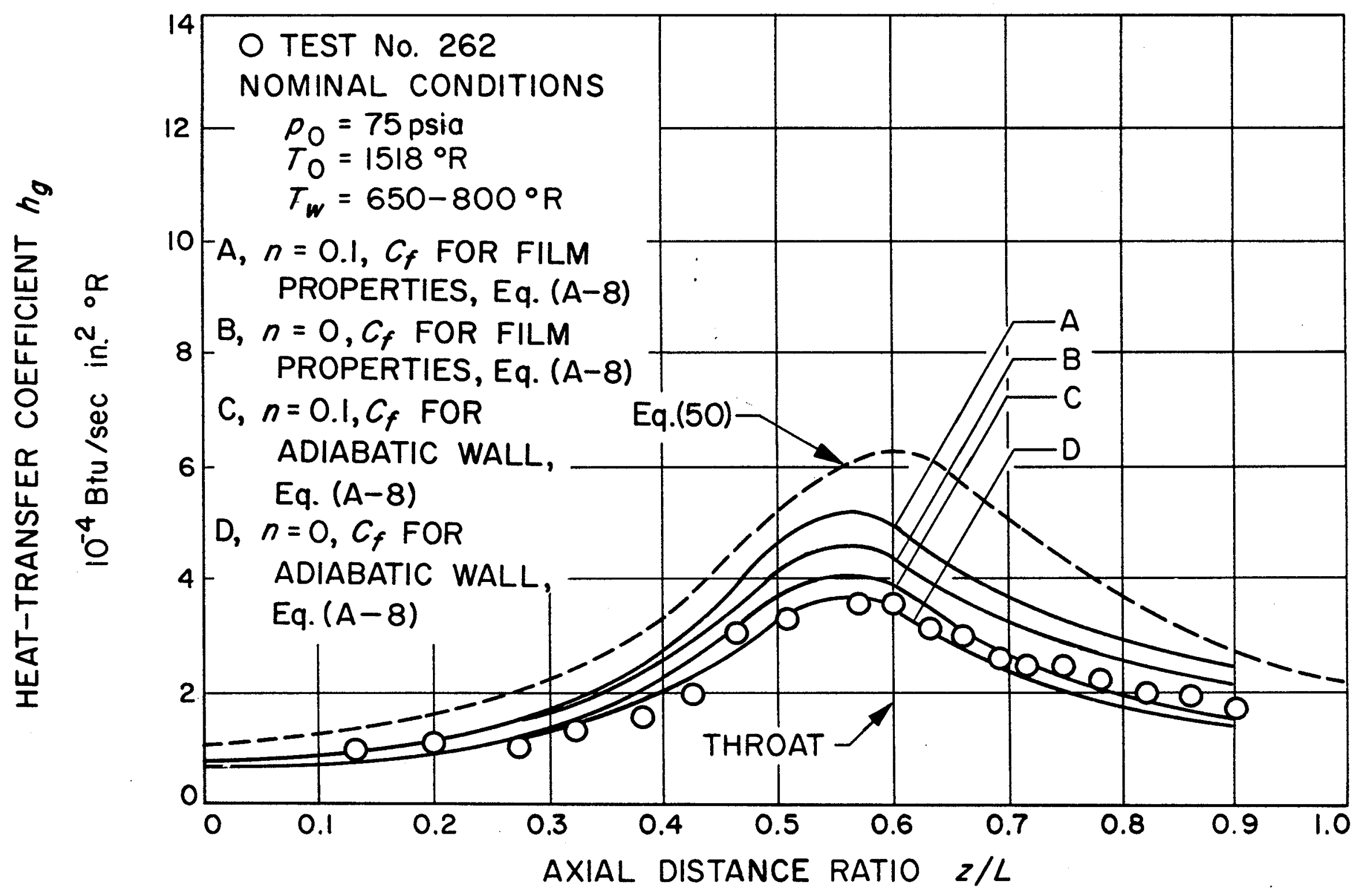




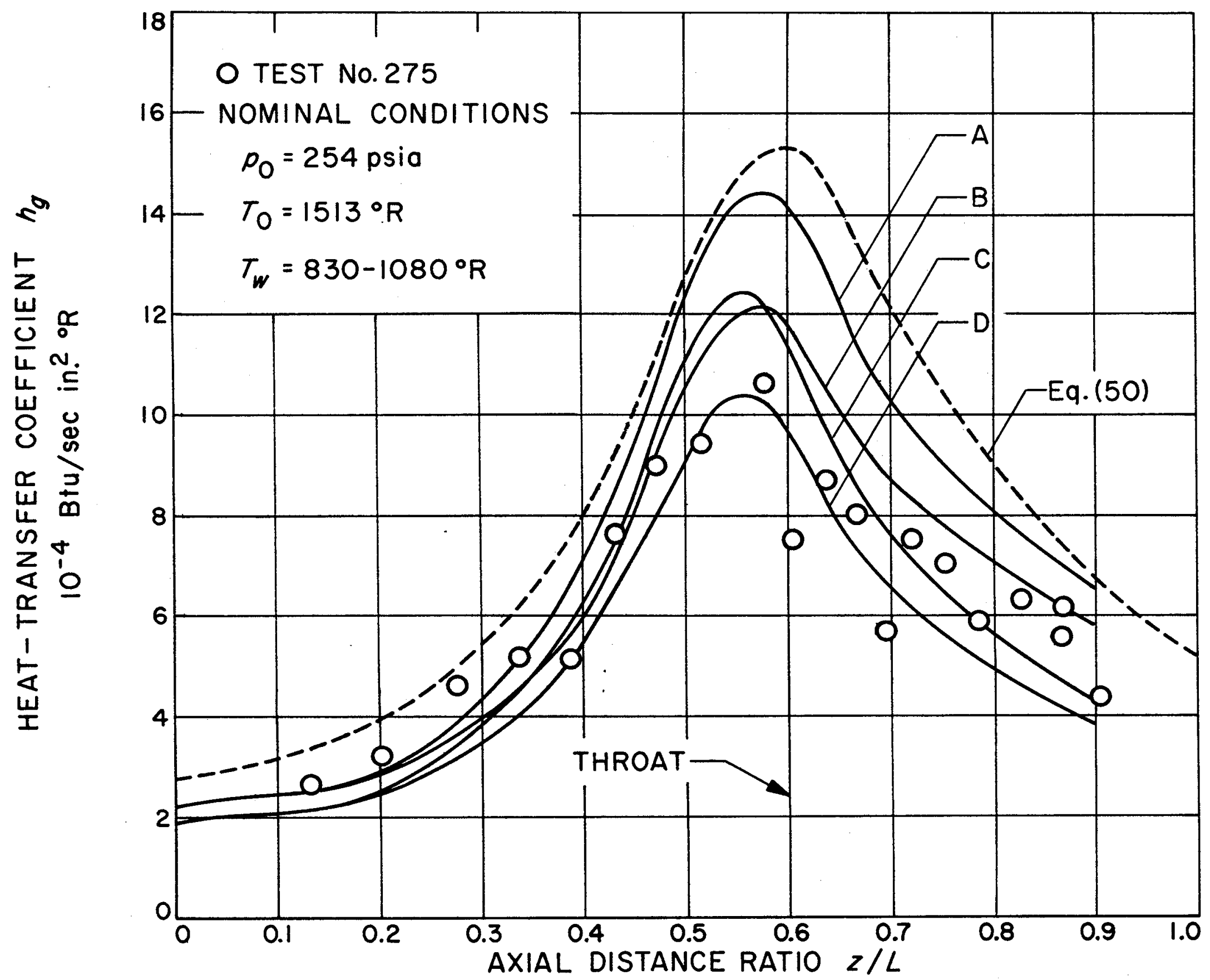




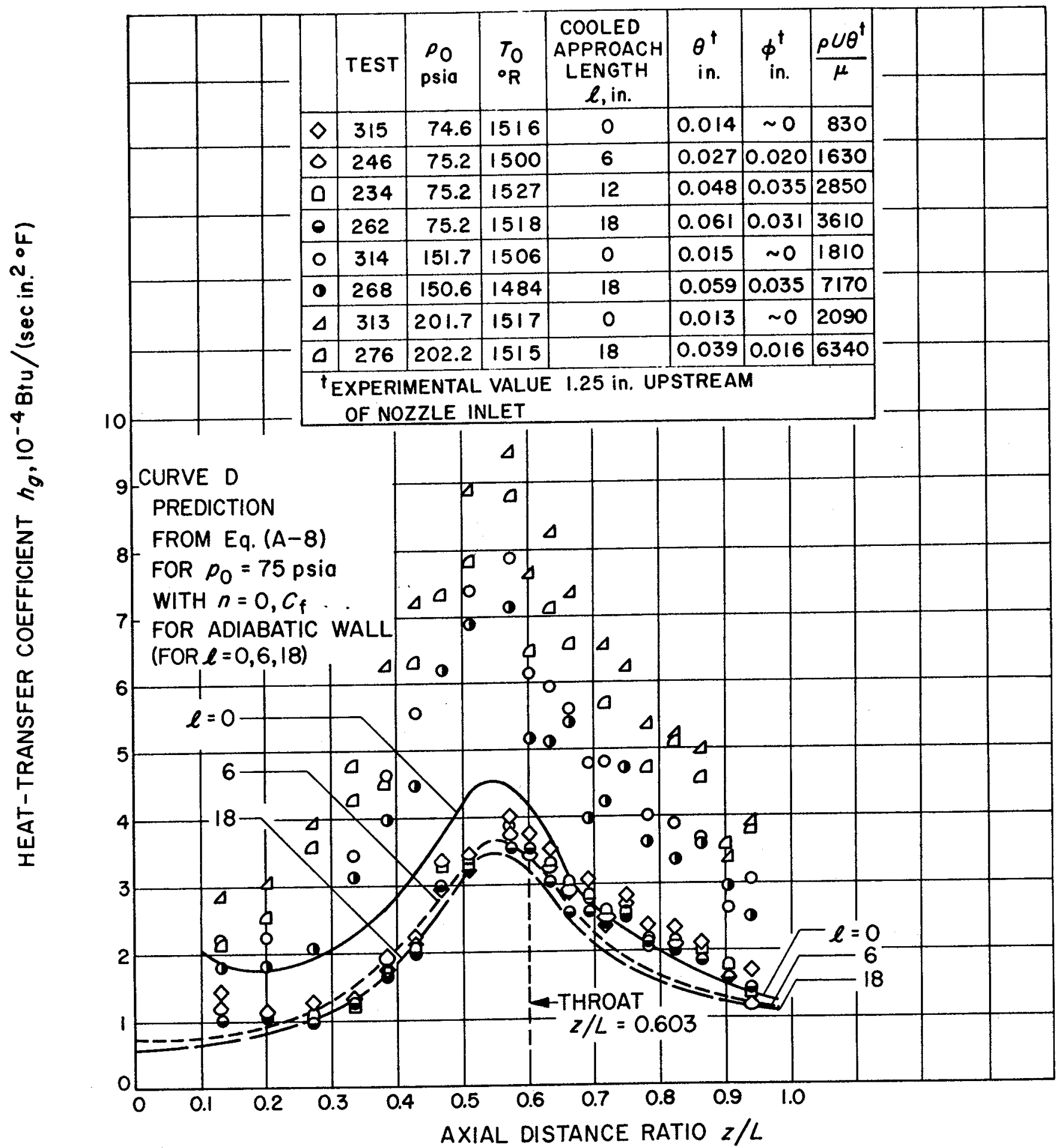




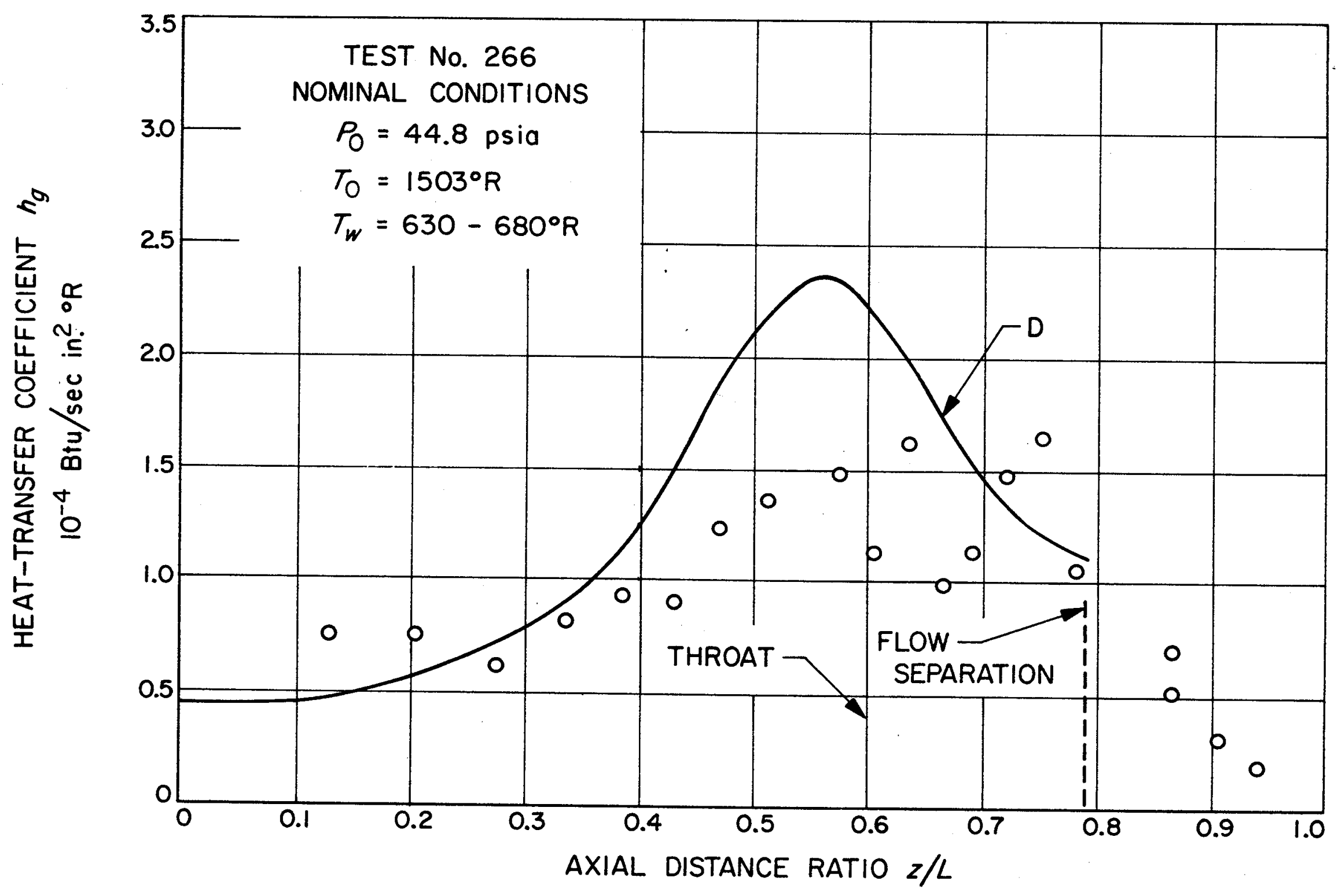


- PREDICTION FROM Eq. (50), $T_{\text {ref }}=T_{a m} ; T_{w} / T_{0}$ AS NOTED

--- PREDICTION FROM Eq. $(48), T_{\text {ref }}=T_{\text {oW }}$

- PREDICTION FROM Eq. (A-8) FOR $T_{0}=1500^{\circ} R, n=0, C_{f}$ FOR ADIABATIC WALL

$\square \quad T_{0} \cong 1030^{\circ} R \quad \gamma=1.380$

$O T_{0} \cong 1500^{\circ} R \quad \gamma=1.345$

$\Delta T_{0} \cong 2000^{\circ} \mathrm{R} \quad \gamma 1.328$
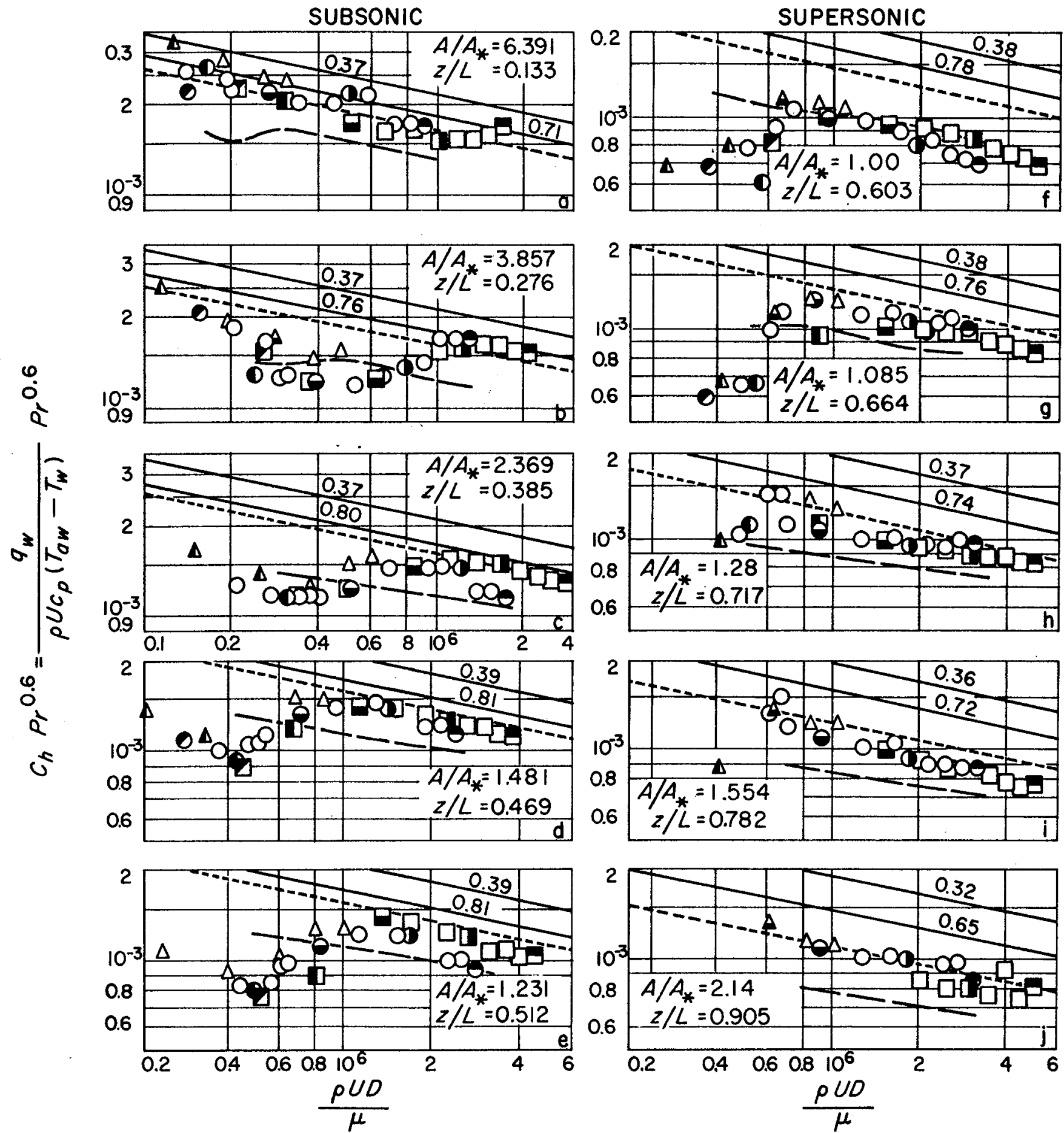


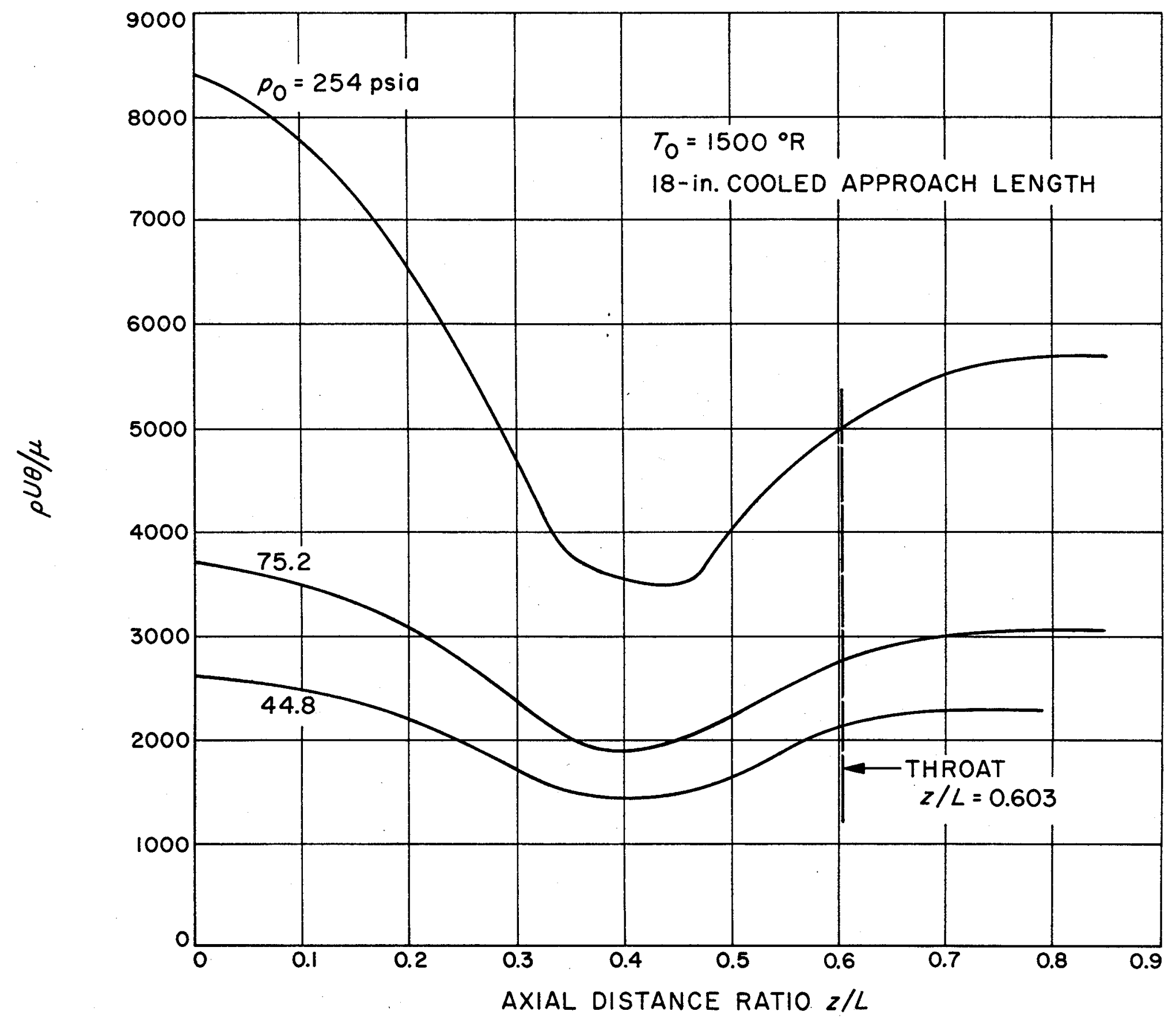




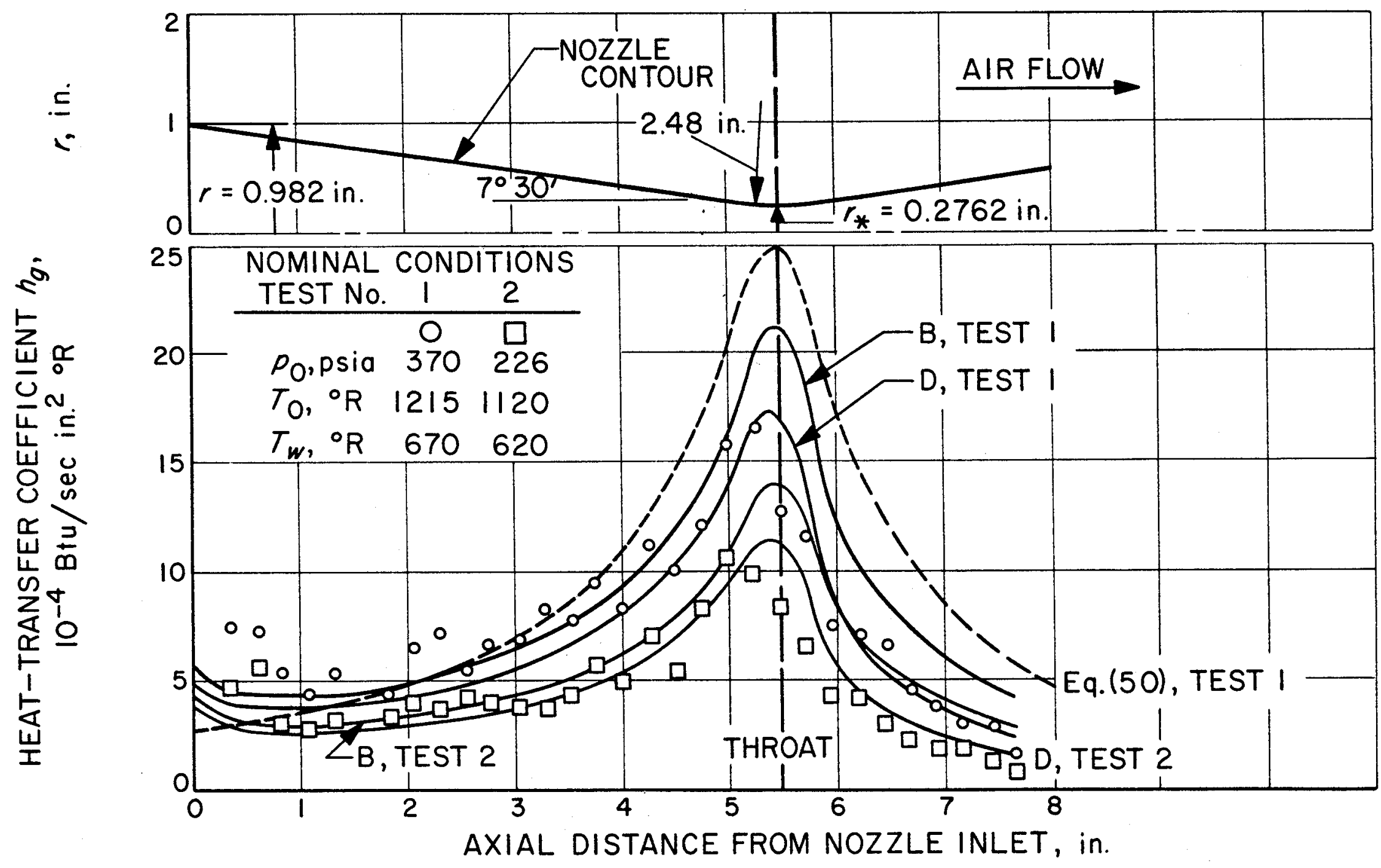



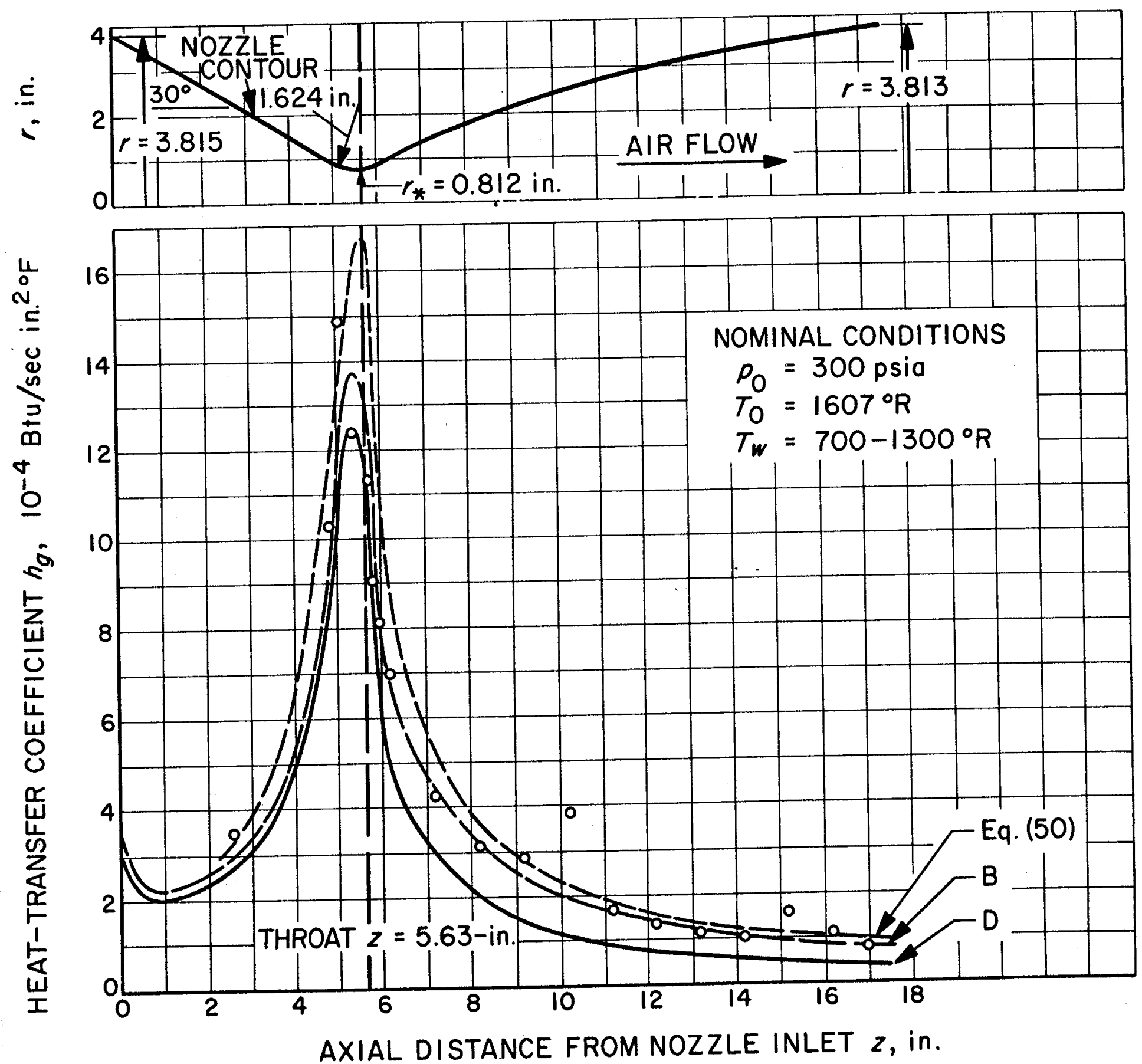


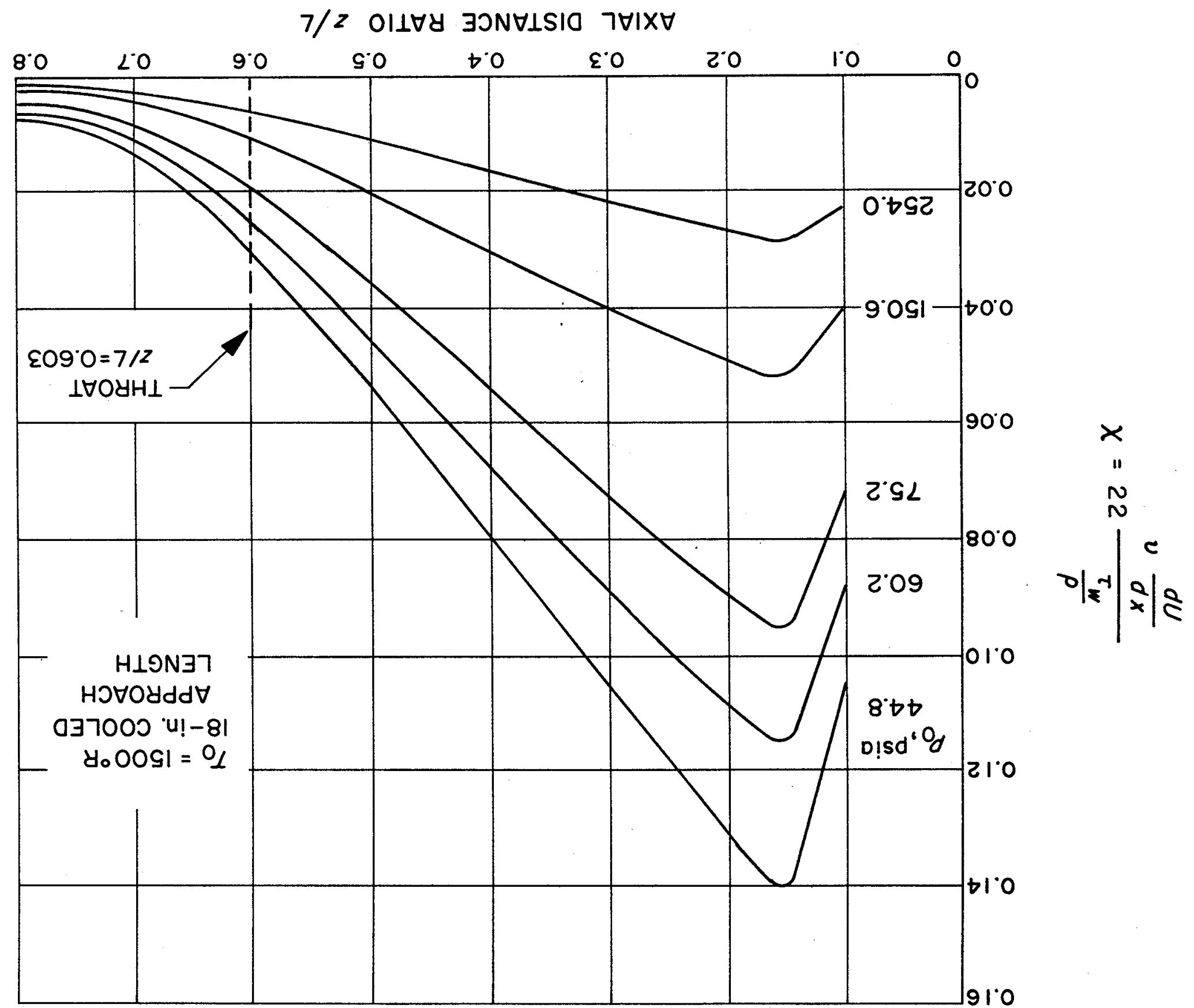


Fis 28

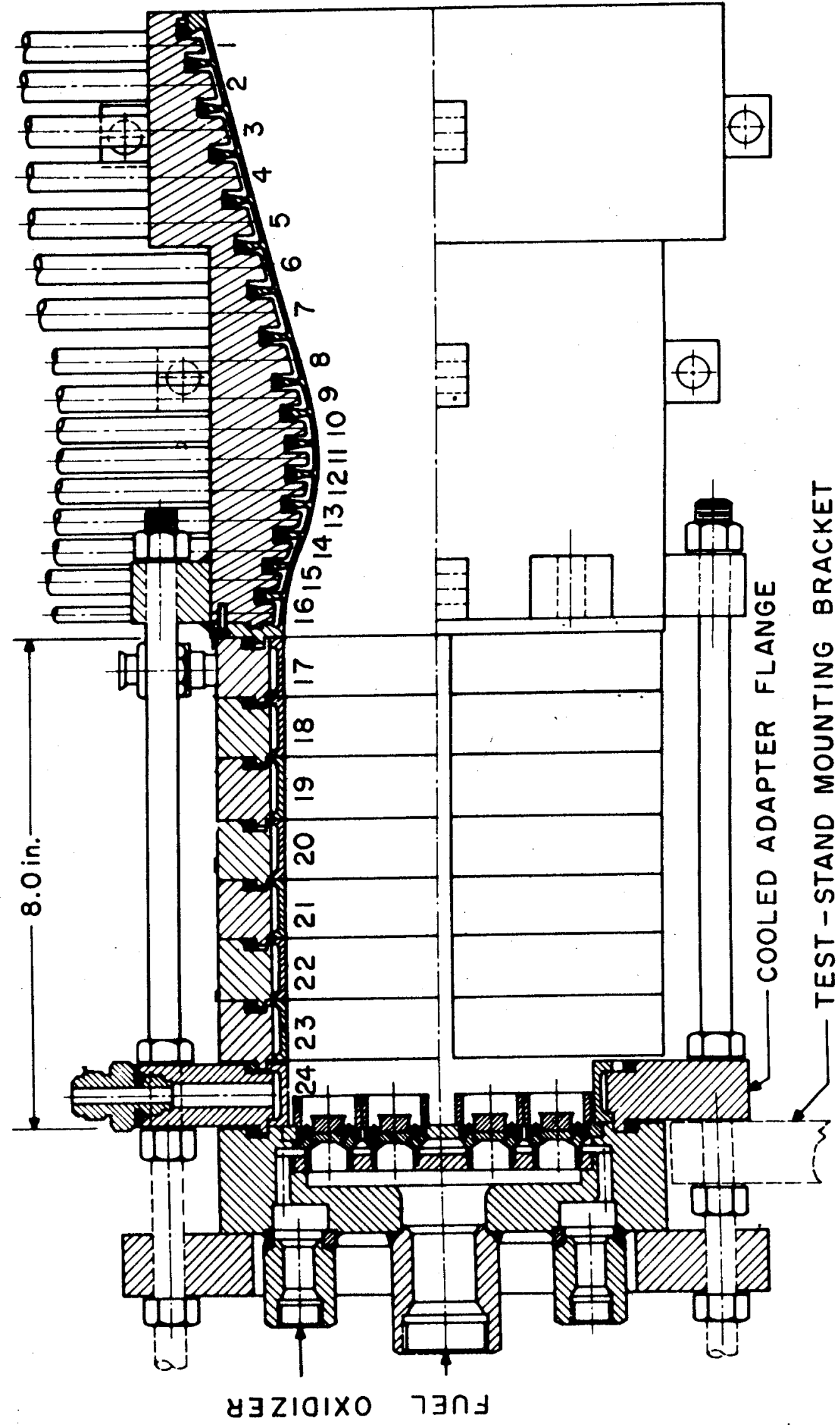




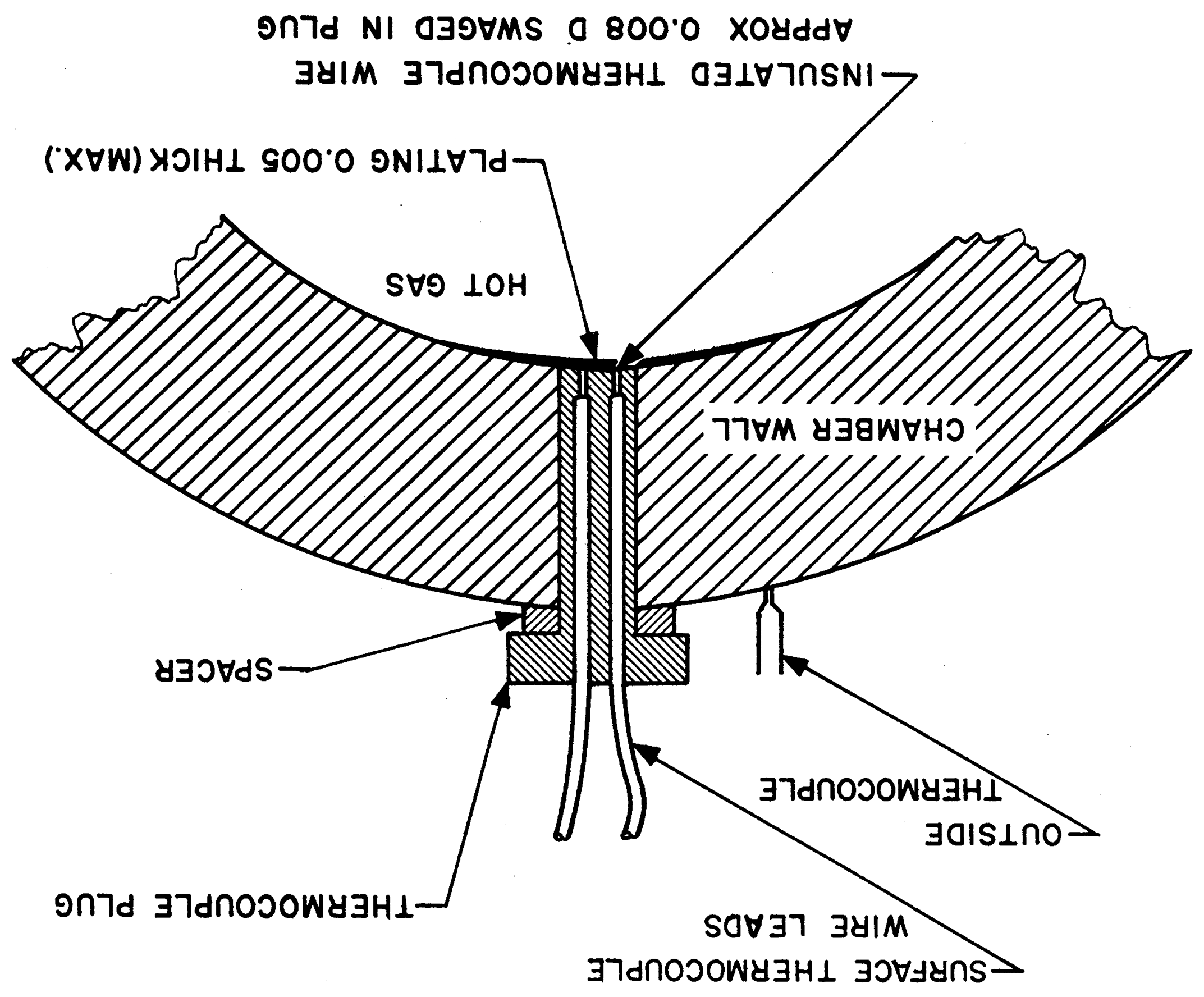




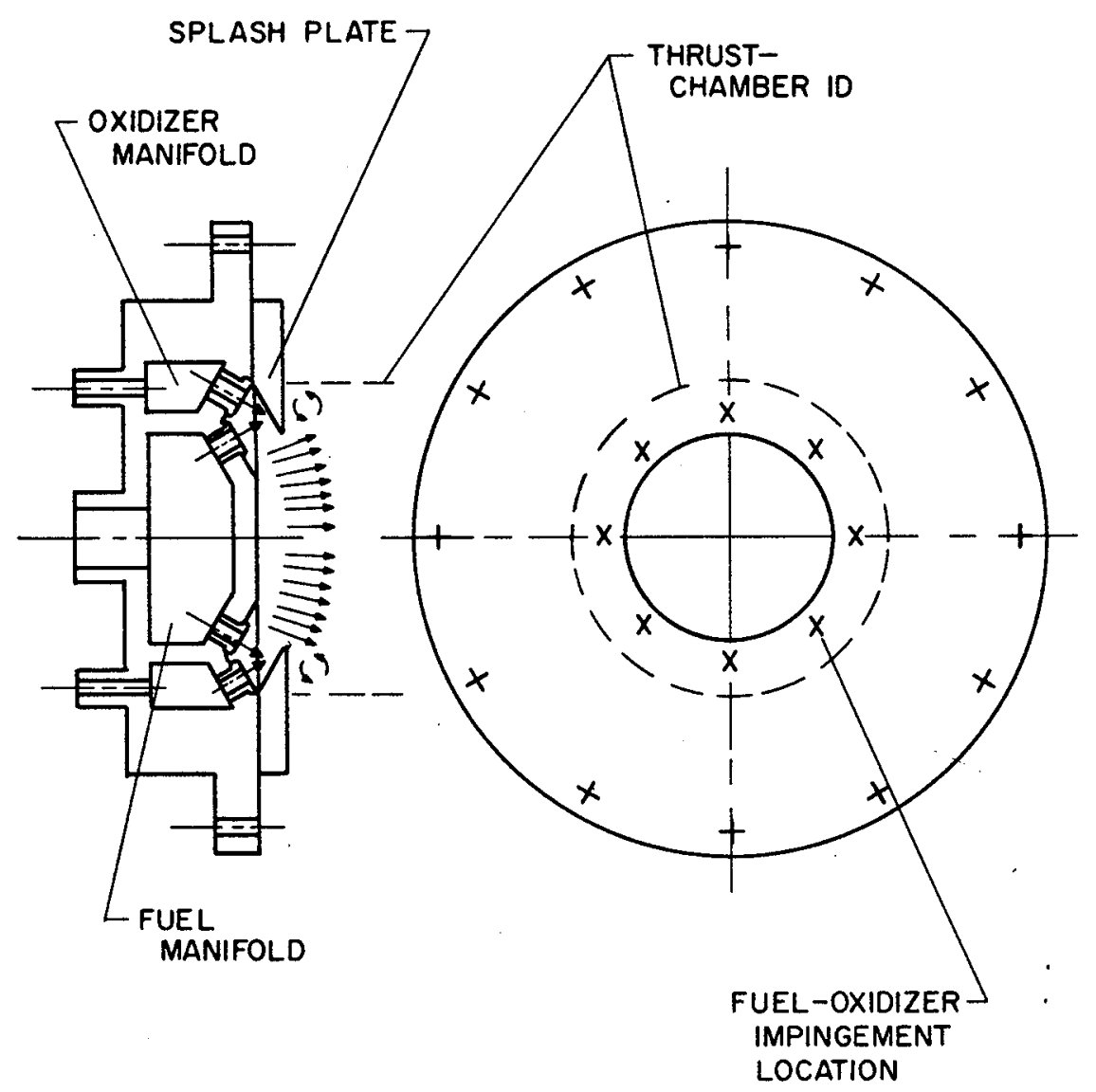

(a) ENZIAN TYPE

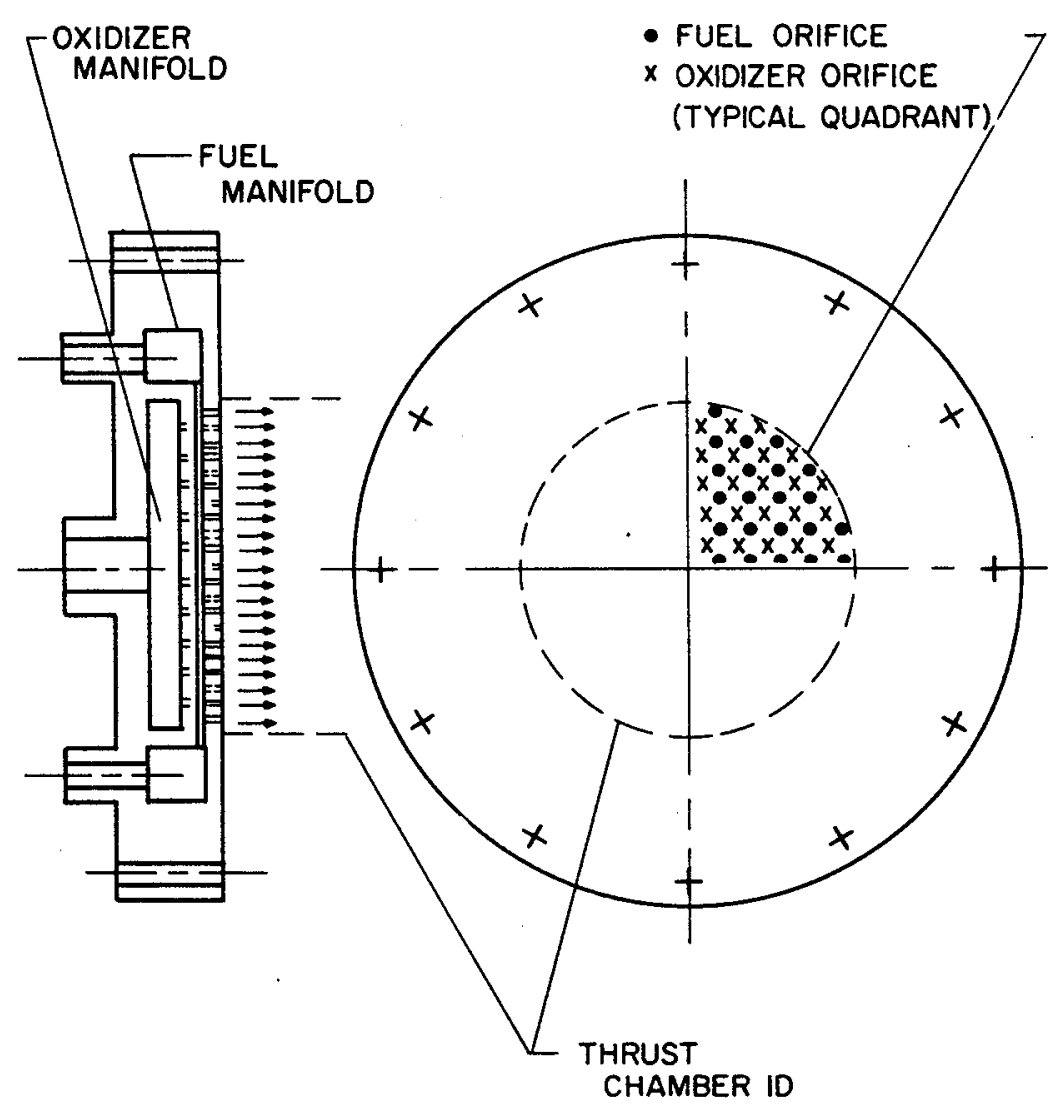

(b) SHOWERHEAD TYPE 

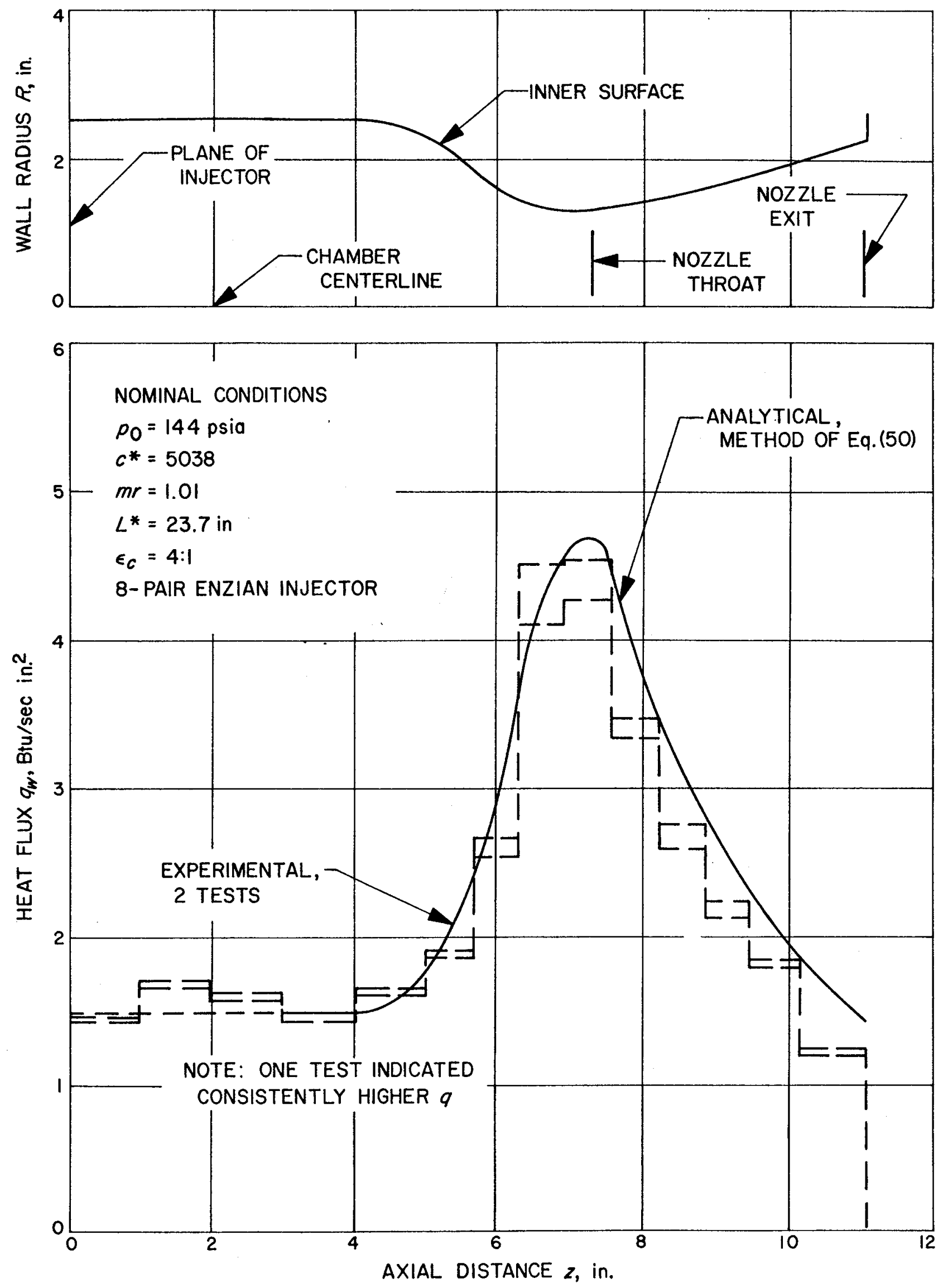

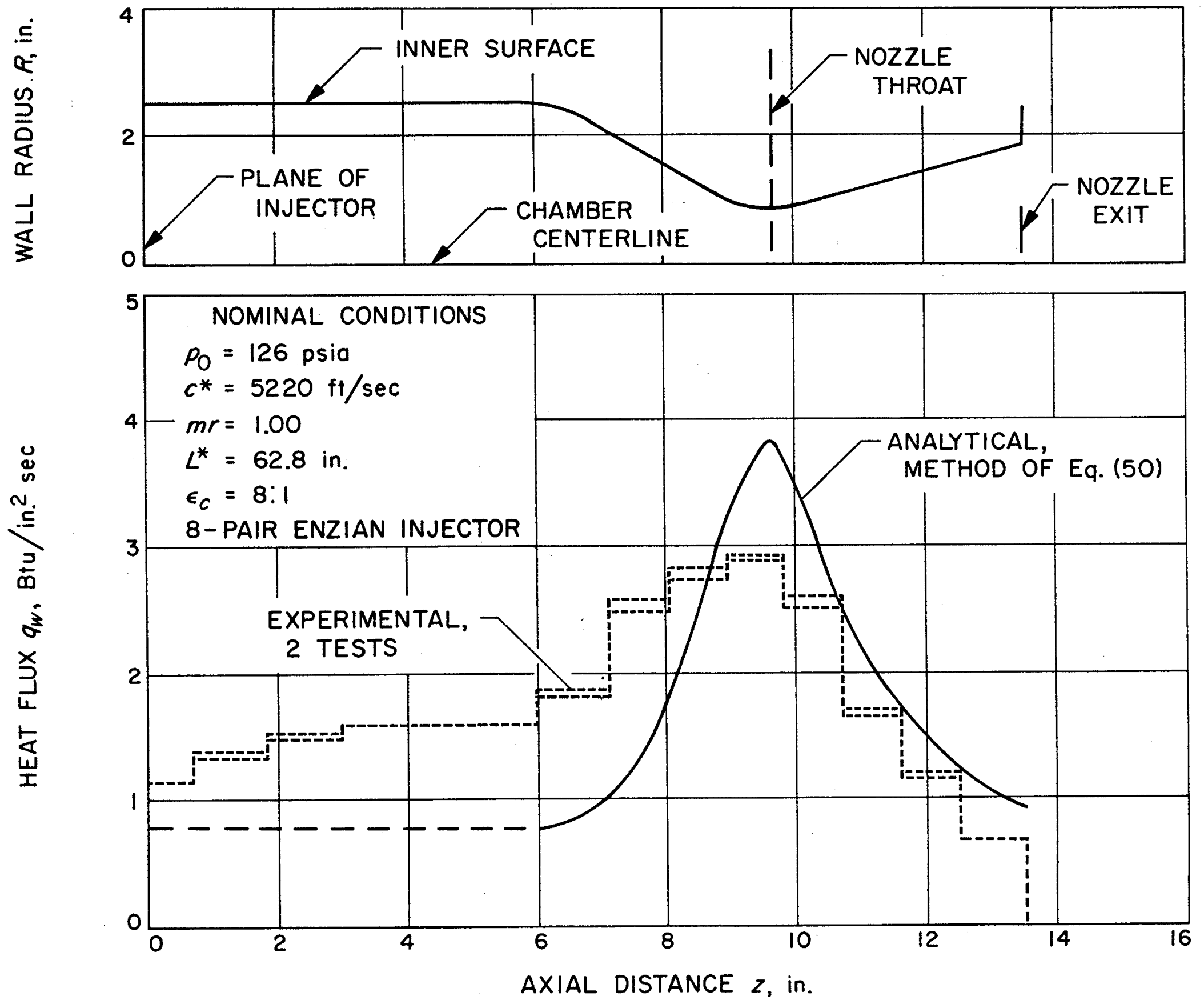

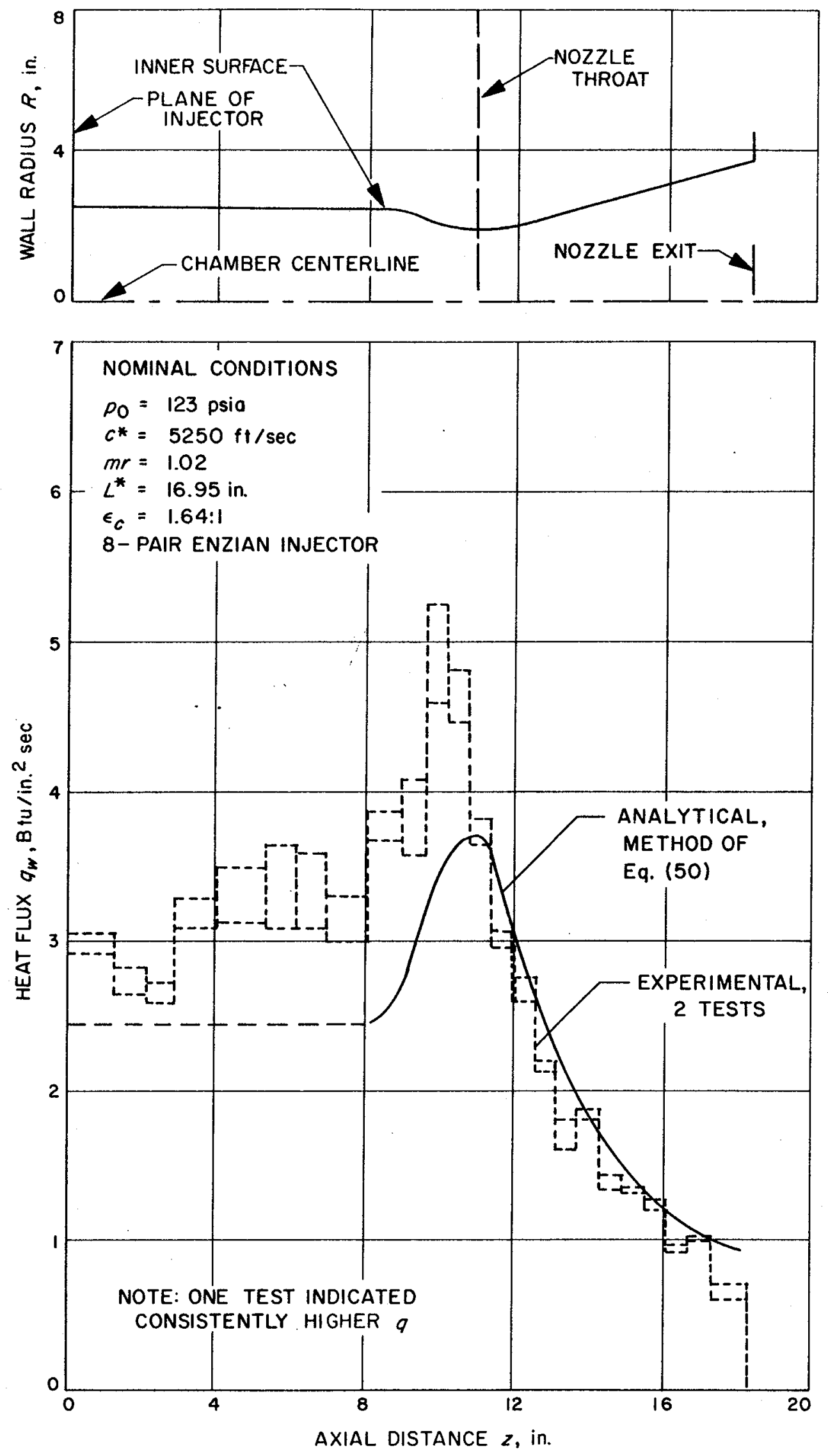


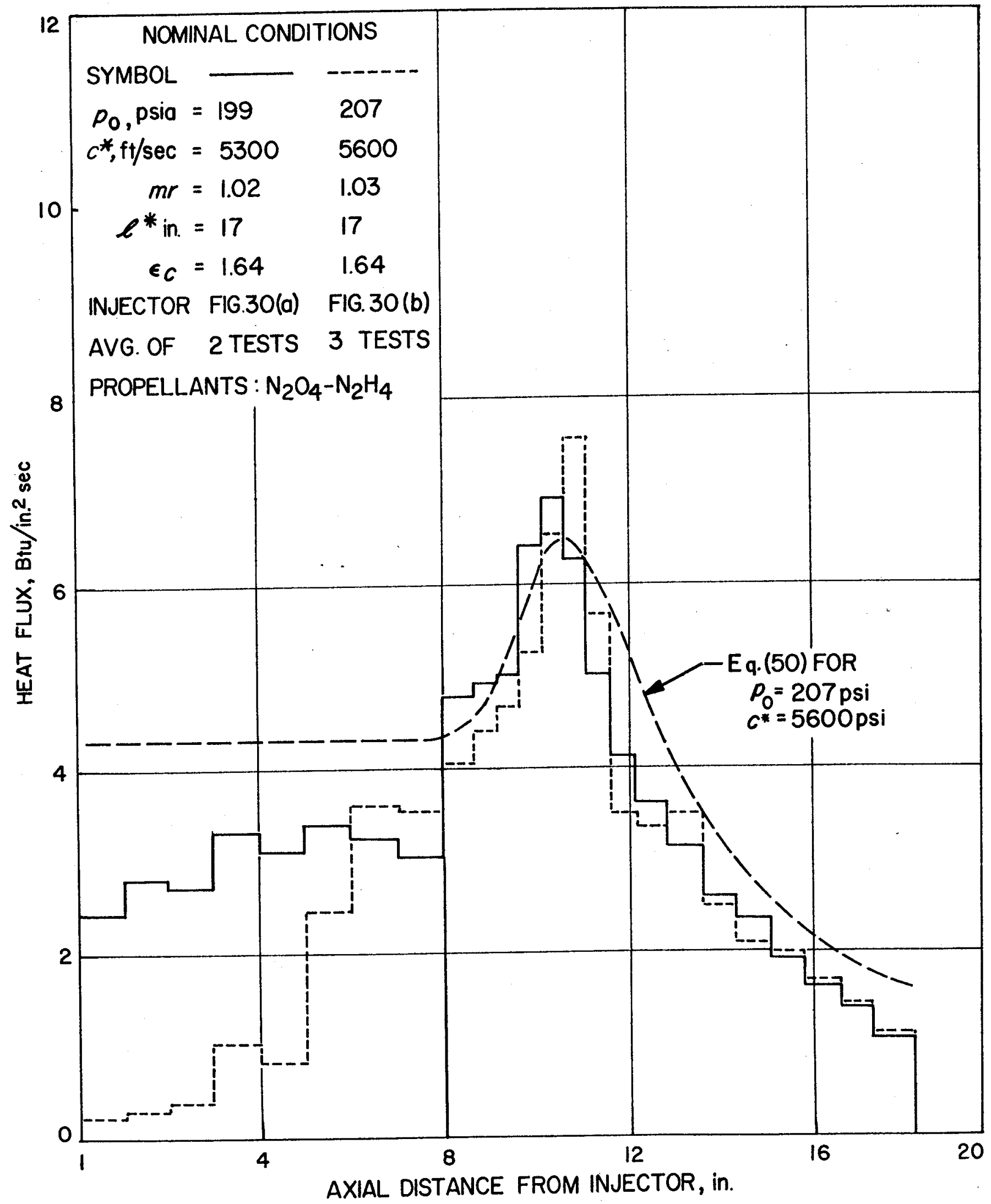



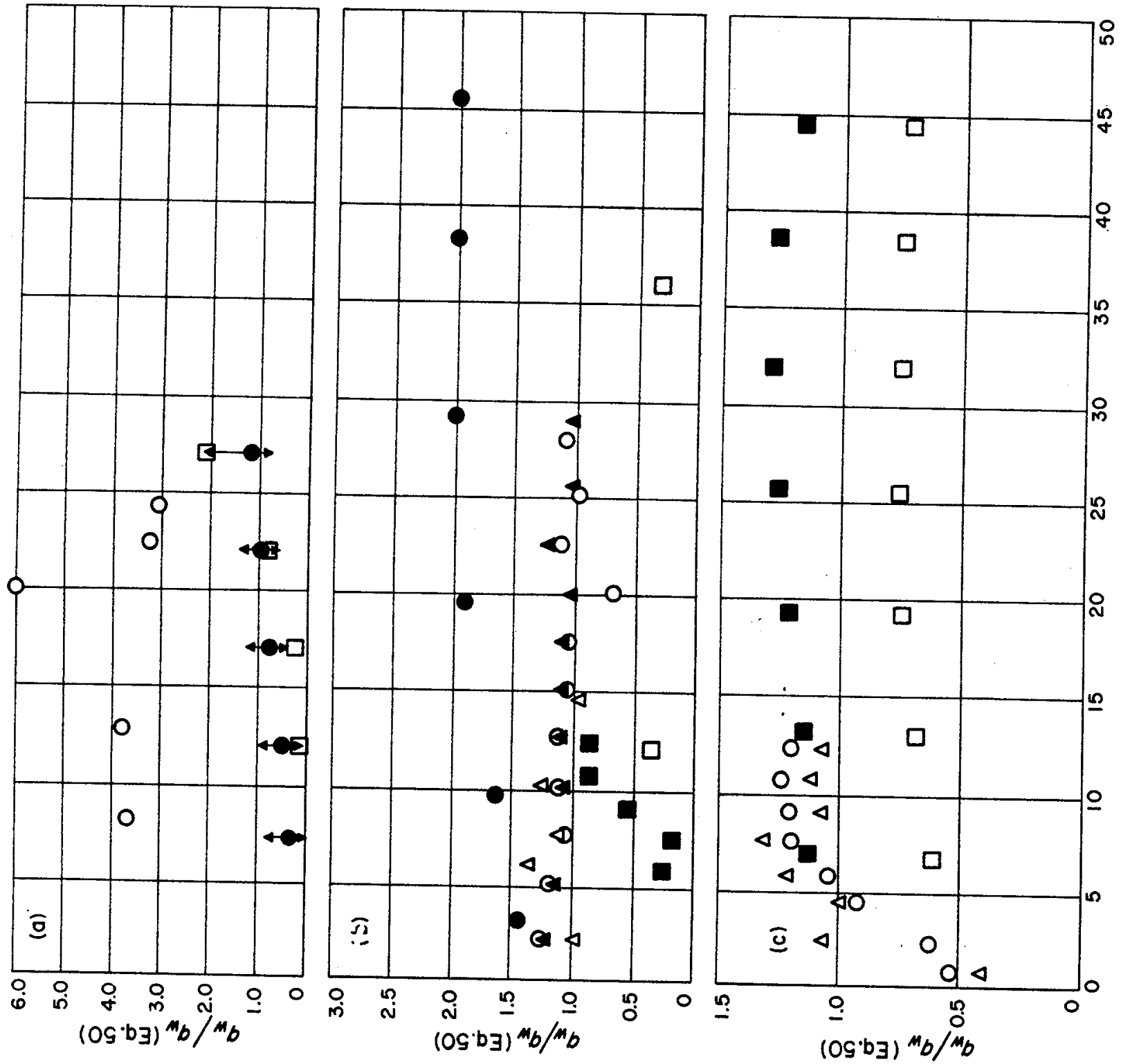

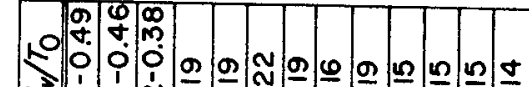
₹ - 0 o 0

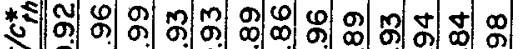
* O.

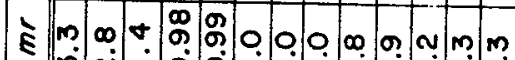

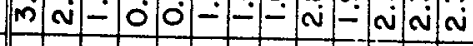

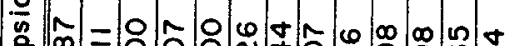

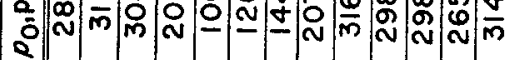
$\dot{s}$

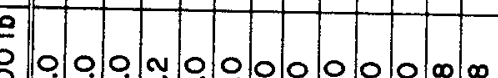
- 일 要 (4)

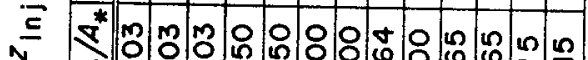

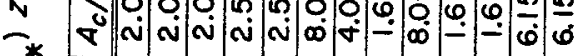
₹* 5

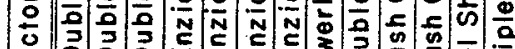
๑

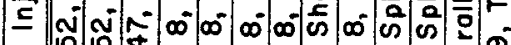

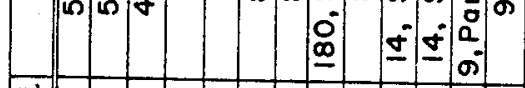

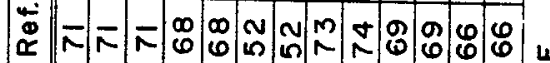

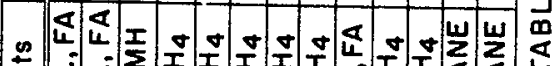
c - 1 z $z z z z<z z a$

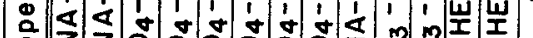
年

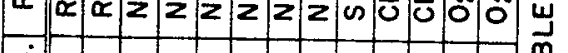

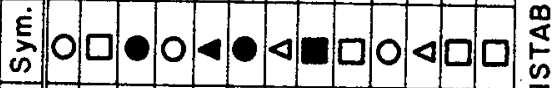

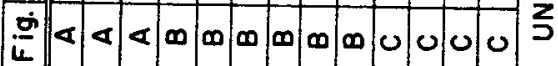




$$
\begin{aligned}
& A_{c} / A_{*}=8 \text { NOZZLE, } L^{*}=63 \mathrm{in} . \\
& \mathrm{N}_{2} \mathrm{O}_{4}-\mathrm{N}_{2} \mathrm{H}_{4} ; m r=0.98-1.03 \\
& c^{*} / c_{\text {th }}^{*}=0.89-0.96
\end{aligned}
$$$$
p_{0}=77-292 \text { psia }
$$

Eq. (50) $-T_{\text {ref }}=T_{a m}, T_{w} / T_{0}=0.18$

Eq. (48) $--T_{\text {ref }}=T_{a w}$

SUBSONIC

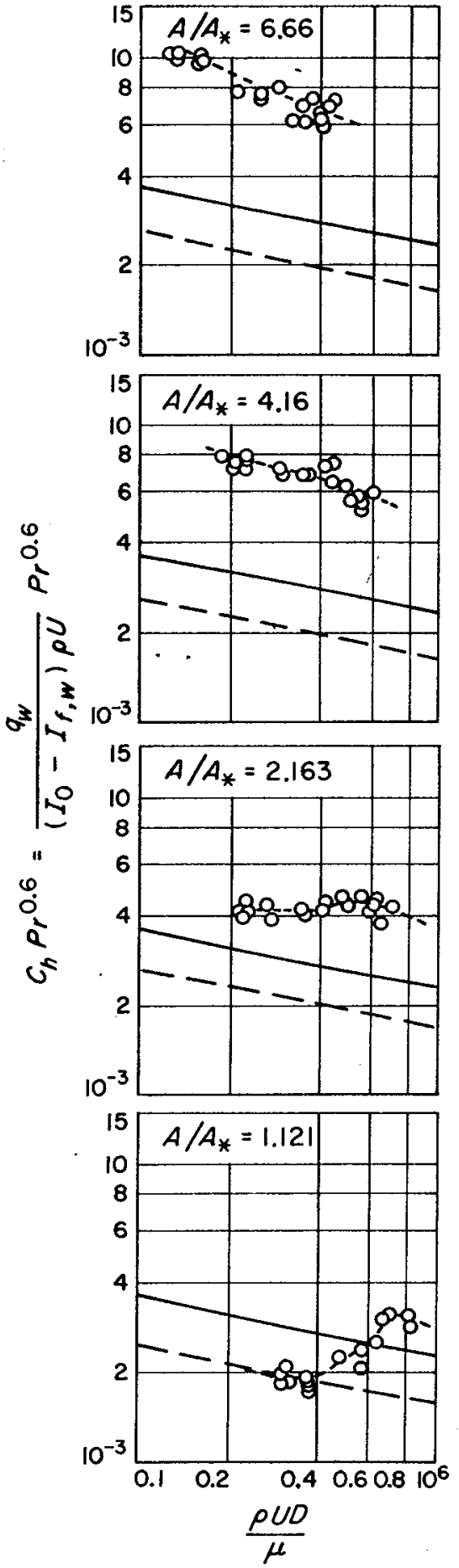

SUPERSONIC
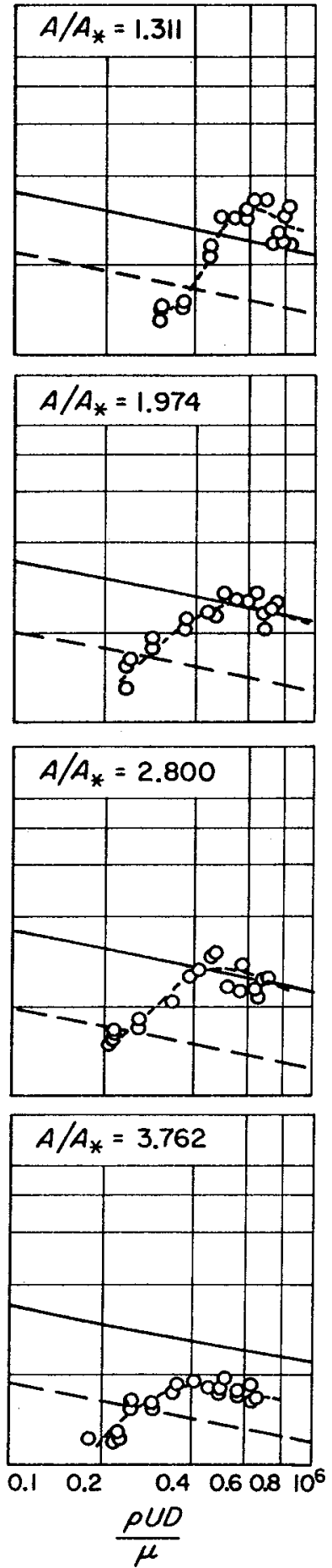


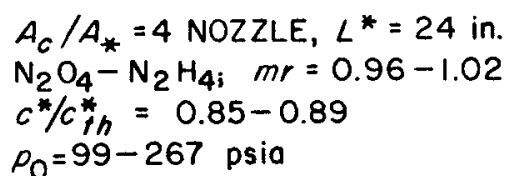

$p_{0}=99-267$ psia

Eq. $(50)-T_{\text {ref }}=T_{a m}, T_{w} / T_{0}=0.20$

Eq. $(48)-T_{\text {ref }}=T_{a w}$
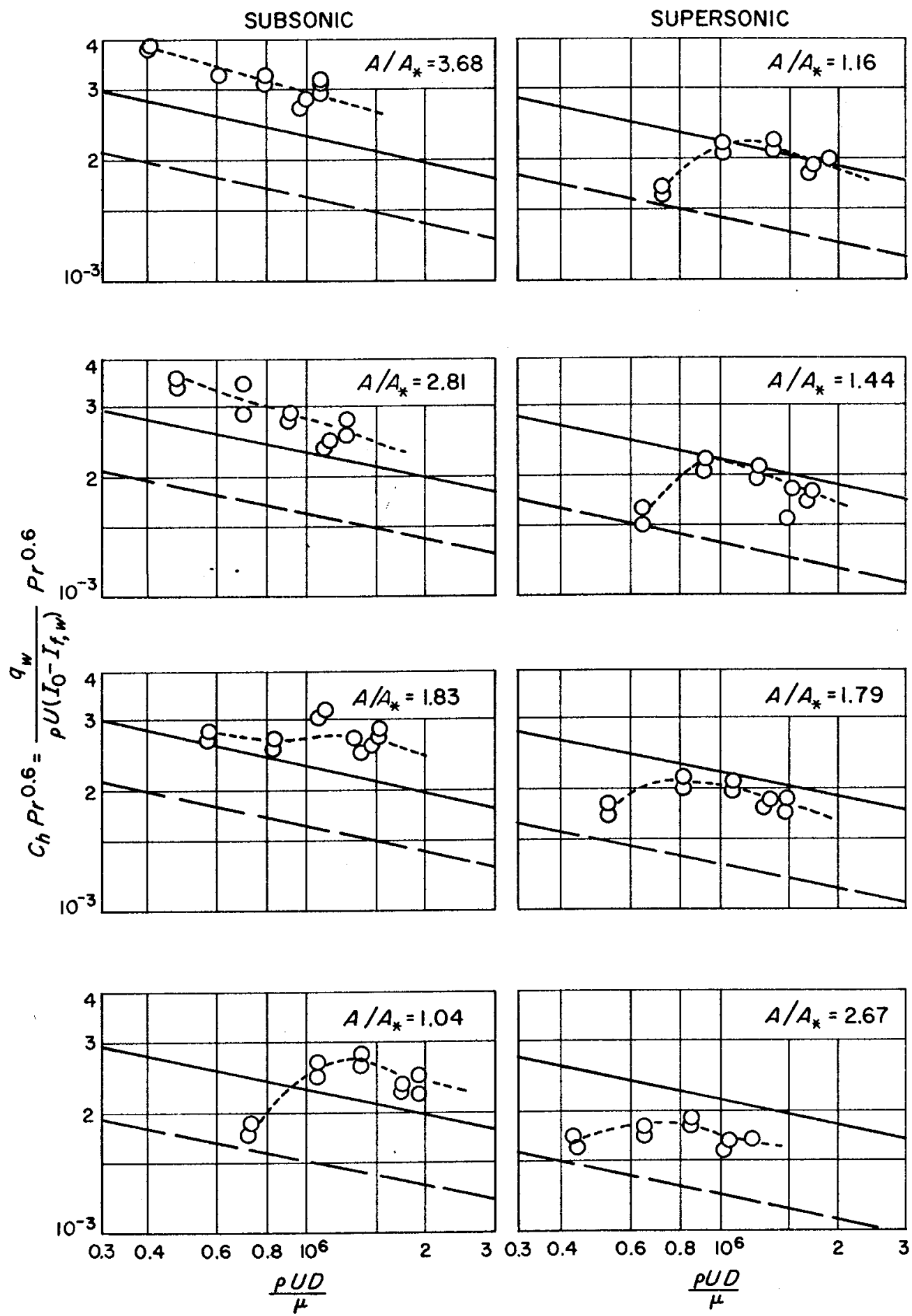


$$
\begin{aligned}
& A_{c} / A_{*}=2.5 \text { NOZZLE, } L^{*}=35.8 \mathrm{in} . \\
& \mathrm{N}_{2} \mathrm{O}_{4}-\mathrm{N}_{2} \mathrm{H}_{4} ; \mathrm{mr}=0.99
\end{aligned}
$$$$
c^{*} / c_{\text {th }}^{*}=0.93-0.94
$$$$
p_{0}=100-301 \text { psia }
$$

$$
\begin{aligned}
& \text { Eq. (50) }-T_{\text {ref }}=T_{a m}, T_{w} / T_{0}=0.20 \\
& \text { Eq. (48)---- } T_{\text {ref }}=T_{a w}
\end{aligned}
$$
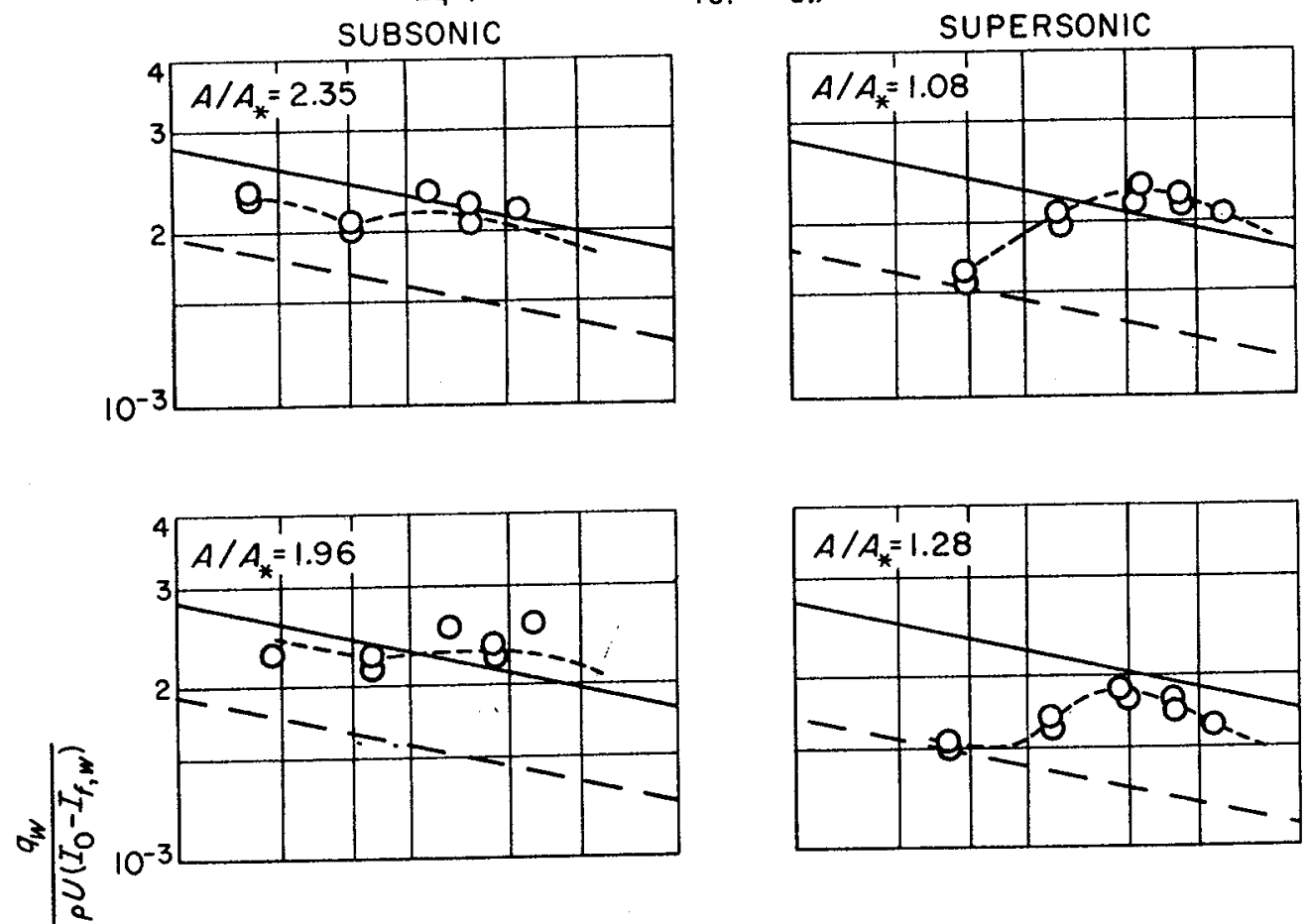

01
0
0
0
0
0
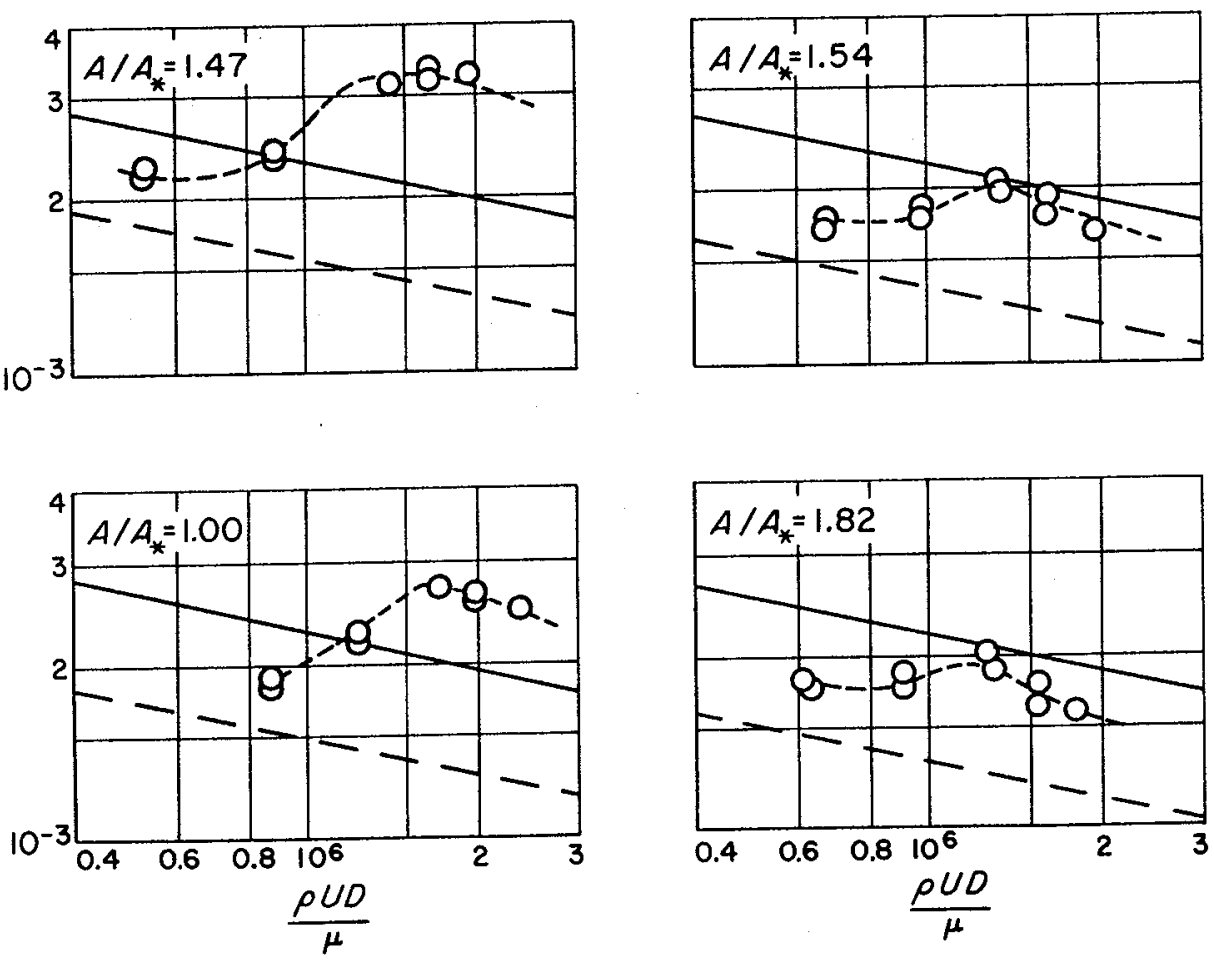
$A_{c} / A_{*}=1.64$ NOZZLE, $L^{*}=17$ in.

$\mathrm{N}_{2} \mathrm{O}_{2}-\mathrm{N}_{2} \mathrm{H}_{4}, m r=0.98$

$c^{*} / c_{t h}^{*}=0.91-0.93$

$p_{0}=97-246$ psia

Eq. $(50)-T_{\text {ref }}=T_{o m}, T_{w} / T_{0}=0.28$

Eq. $(48)--T_{\text {ref }}=T_{\text {ow }}$
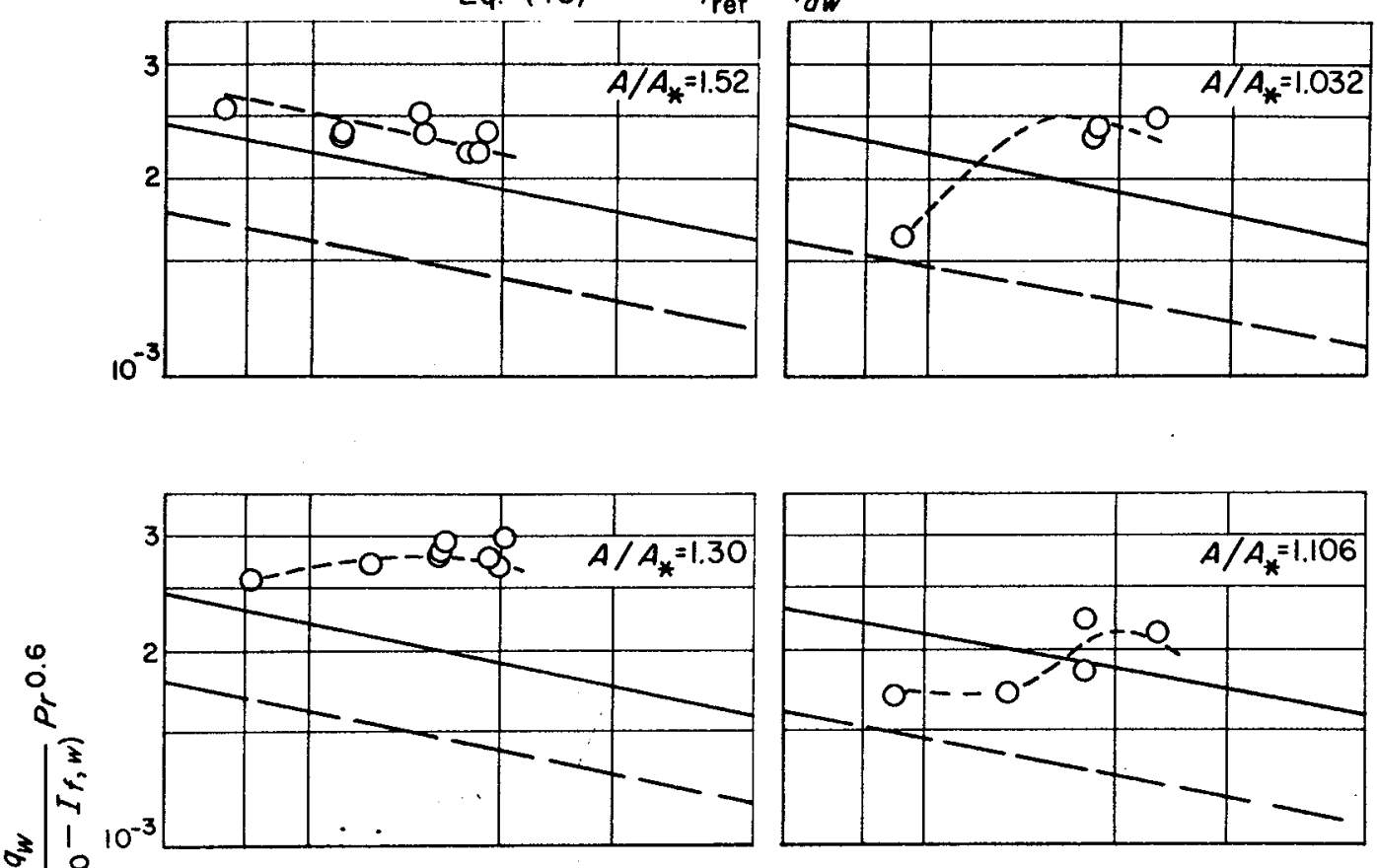

$\sigma$

No

Ia
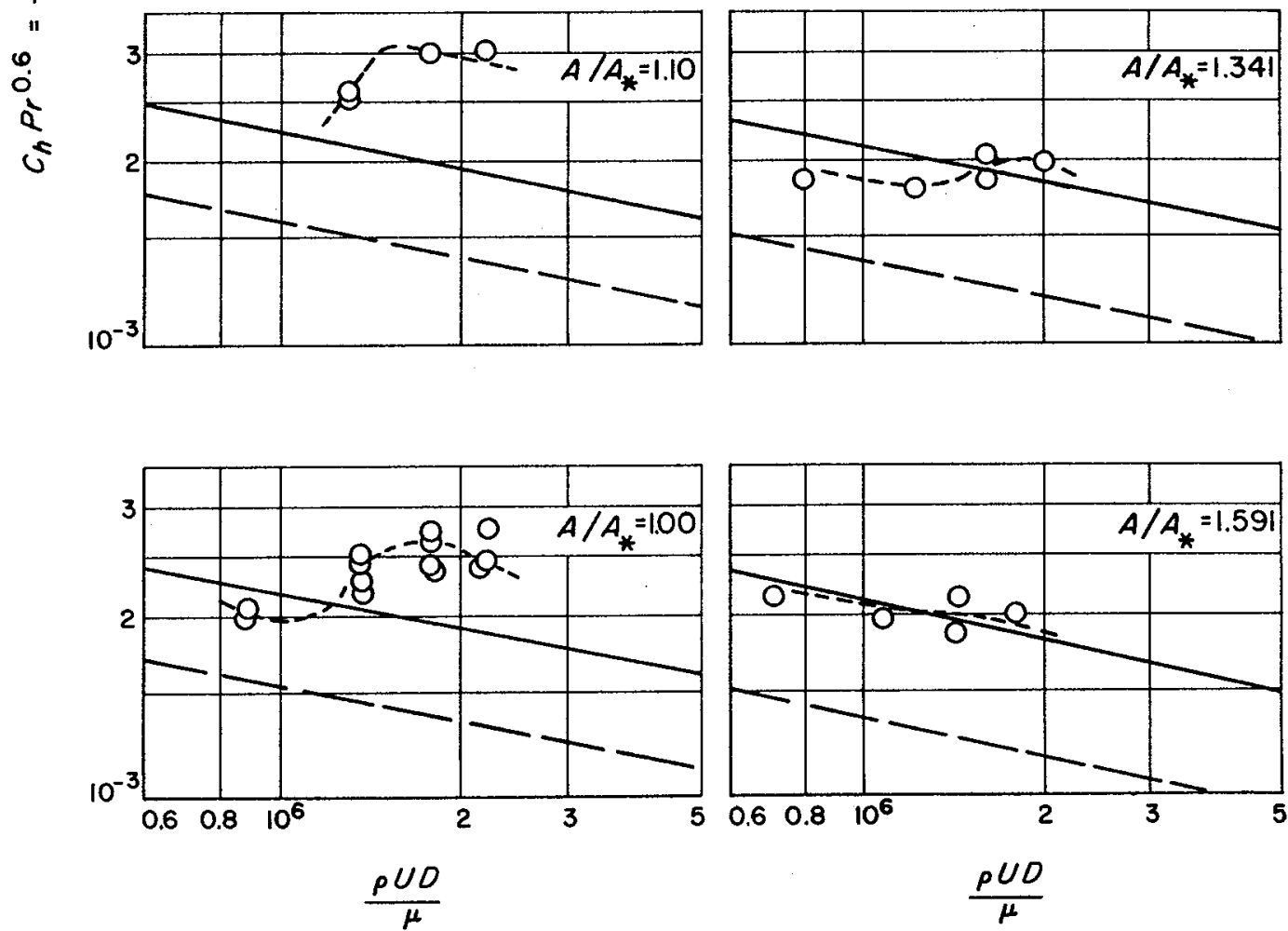

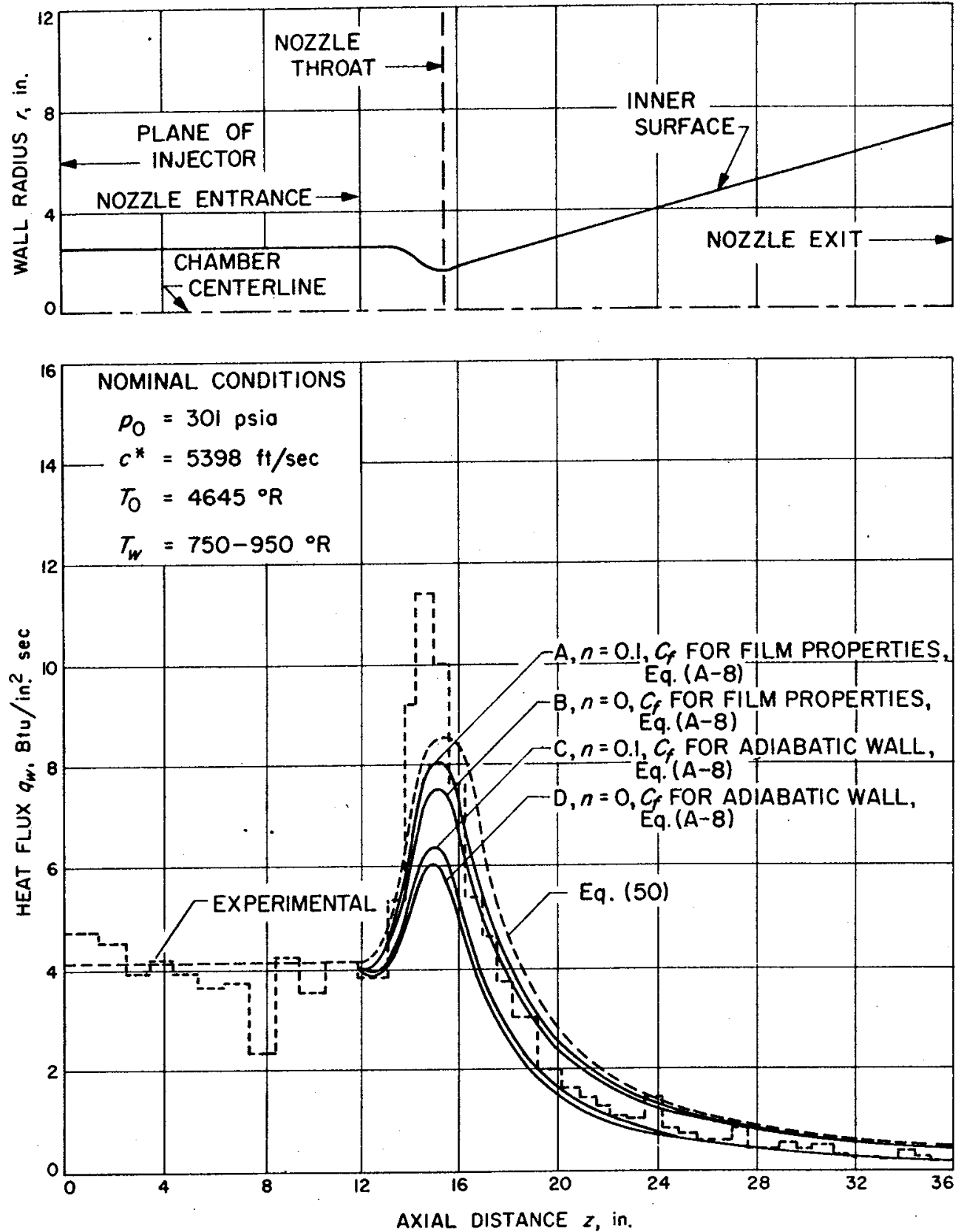

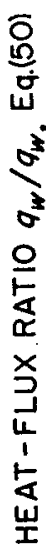

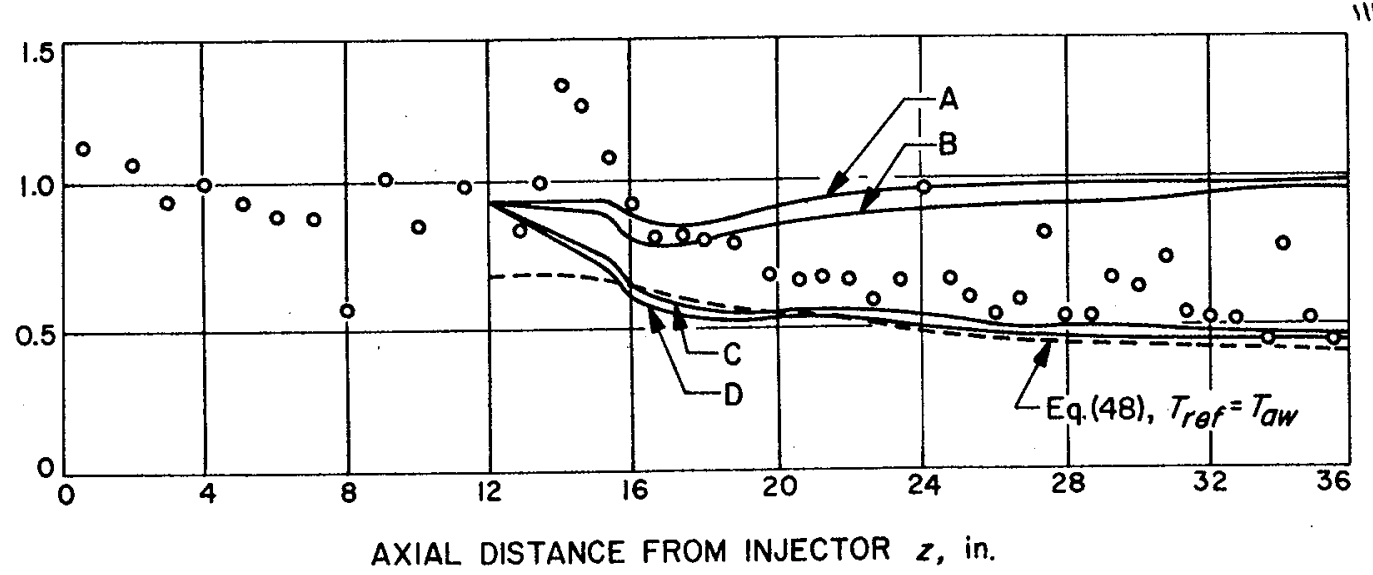

